\title{
Sistemas Impulsivos com Retardamento: Soluções Periódicas
}

\author{
Selma Helena de Jesus Nicola \\ Orientador:
}

Prof. Dr. Plácido Zoega TÁBOAS

Co-orientador:

Prof. Dr. Luiz Augusto da Costa Ladeira

Tese de Doutorado apresentada ao Instituto de Ciências Matemáticas e de Computação, da Universidade de São Paulo, como parte dos requisitos para obtenção do título de "Doutor em Ciências - Área: Matemática".

\section{USP - São Carlos}

Agosto de 2000 
Dedico a meu pai (in memorian),

a meu esposo Aldemir e minha família. 


\section{Agradecimentos}

Ao Prof. Dr. Plácido Zoéga Táboas e ao Prof. Dr. Luiz Augusto da Costa Ladeira pela excelente orientação, paciência e amizade.

Aos professores e colegas do Departamento de Matemática do ICMC-USP e do Departamento de Matemática da Universidade Federal de São Carlos pela amizade e apoio nos momentos difíceis.

Às funcionárias da Secretaria de Pós-Graduação e da Biblioteca pela simpatia, atenção e eficiência.

À minha família pelo carinho e incentivo.

Enfim, a todos que direta ou indiretamente contribuíram para a realização deste trabalho. 


\begin{abstract}
We prove the existence of periodic solutions of some retarded functional differential equations subjected to impulsive self-supporting conditions. Due to the impulses, solutions exhibit discontinuites of the first kind and this forces the consideration of more general phase spaces than $C\left([-r, 0], \mathbb{R}^{n}\right)$.

We show that periodic solutions can emanate from the origin through local Hopf bifurcations but we also state an existence theorem for slowly spiralling periodic solutions. This theorem is accomplished by a combination of the self-supporting condition with the ejectivity of the origin with respect to a return mapping.
\end{abstract}




\section{Resumo}

Provamos a existência de soluções periódicas de algumas equações diferenciais funcionais com retardamento sujeitas a condições de impulsos de auto-sustentação. Devido aos impulsos, soluções exibem descontinuidades de primeira espécie e isso força considerarmos espaços de fase mais gerais que $C\left([-r, 0], \mathbb{R}^{n}\right)$.

Mostramos que soluções periódicas podem emanar da origem através de bifurcações locais de Hopf, mas também estabelecemos um teorema de existência de soluções periódicas lentamente espiralantes. Esse teorema é obtido combinando-se a condição de auto-sustentação com a ejetividade da origem em relção a uma aplicação de retorno. 


\section{Sumário}

Introdução 1

1 Fatos básicos de uma teoria geral em $L^{p} \quad 4$

1.1 Introdução . . . . . . . . . . . . . . . . . . . . . . 4

1.2 Definições preliminares . . . . . . . . . . . . . . . . 4

1.3 Existência . . . . . . . . . . . . . . . . . 5

1.4 Dependência contínua .................. 20

1.5 Unicidade . . . . . . . . . . . . . . . . . . 24

1.6 Continuação de soluções . . . . . . . . . . . . . . . . . . 27

1.7 Comentários finais . . . . . . . . . . . . . . 35

2 Equações características e bifurcações de Hopf 36

2.1 Introdução . . . . . . . . . . . . . . . . . . 36

2.2 Uma motivação escalar . . . . . . . . . . . . . . . . . . . . 37

2.3 Uma equação planar . . . . . . . . . . . . . . . . . . . . . . . 41

3 Soluções periódicas de um sistema impulsivo 58

3.1 Introdução . . . . . . . . . . . . . . . . . . . 58

3.2 Soluções lentamente espiralantes . . . . . . . . . . . . . . . . 60

3.3 Comentários finais . . . . . . . . . . . . . . . 75

$\begin{array}{ll}\text { Referências Bibliográficas } & 77\end{array}$ 


\section{Introdução}

Um procedimento usual para o estudo da existência de soluções periódicas de uma equação diferencial funcional com retardamento é uma idéia devida a Poincaré que tem origem na teoria dos Sistemas Dinâmicos. Trata-se de determinar um subcon-

junto $K$ do espaço de fase e definir um operador de retorno $A: K \rightarrow K$, seguindo as órbitas positivas a partir de condições iniciais $\varphi \in K$. Os pontos fixos de $A$ corresponderão a soluções periódicas.

Este método depende da existência de um conjunto limitado positivamente invariante, o que é em geral garantido se o segundo membro da equação contém um termo dissipativo.

Nosso objetivo é aplicar essas idéias ao estudo de um sistema planar de equações diferenciais funcionais com retardamento. A principal inspiração vem de alguns resultados de Nussbaum [9], sobre a equação escalar

$$
\dot{x}(t)=-\frac{\alpha}{\delta} \int_{-1}^{-1+\delta} f(x(t+\theta)) d \theta
$$

onde $\alpha$ é um número estritamente positivo, $0<\delta \leq 1 / 2, f: \mathbb{R} \rightarrow \mathbb{R}$ é contínua, $f^{\prime}(0)=1, x f(x)>0$ se $x \neq 0$, e $f(x) \geq-B>-\infty$ se $x \in \mathbb{R}$.

Mais precisamente, queremos estabelecer alguma contrapartida bidimensional de parte desses resultados. Em nosso caso vamos introduzir uma condição de impulso para desempenhar o papel da dissipatividade para garantir a existência de um conjunto limitado positivamente invariante.

Um sistema impulsivo é uma equação diferencial sujeita a uma condição adi- 
cional que reflete perturbações de duração tão curta que podem ser consideradas instantâneas, isto é, impulsos.

Segundo Lakshmikantham [6], um sistema impulsivo pode ser representado, no caso ordinário, por

(i) uma equação diferencial

$$
\dot{x}=f(t, x)
$$

onde $f: \mathbb{R}_{+} \times \Omega \rightarrow \mathbb{R}^{n}, \Omega \subset \mathbb{R}^{n}$ aberto, satisfaz alguma condição de unicidade para problemas de valores iniciais,

(ii) subconjuntos $M(t) \subset \Omega, t \in \mathbb{R}_{+} \mathrm{e}$

(iii) funções $A(t): M(t) \rightarrow \Omega, t \in \mathbb{R}_{+}$.

Uma solução desse sistema pelo ponto $\left(t_{0}, x_{0}\right) \in \mathbb{R}_{+} \times \Omega$ coincide com a solução $x(t)=x\left(t ; t_{0}, x_{0}\right)$ de $(2)$ por $\left(t_{0}, x_{0}\right)$ para $t \in\left(t_{0}, t_{1}\right]$, onde $t_{1}$ é o menor valor de $t>t_{0}$ com $x\left(t_{1}\right) \in M\left(t_{1}\right)$. Então a função $A\left(t_{1}\right)$ leva $x\left(t_{1}\right)$ em $x_{1} \stackrel{\text { def }}{=} A\left(t_{1}\right) x\left(t_{1}\right)$. A partir deste instante, a solução coincide com a solução $x(t)=x\left(t ; t_{1}, x_{1}\right)$ de $(2)$ por $\left(t_{1}, x_{1}\right)$ para $t \in\left(t_{1}, t_{2}\right]$, onde $t_{2}$ é o menor valor de $t>t_{1} \operatorname{com} x\left(t_{2}\right) \in M\left(t_{2}\right)$. Então a função $A\left(t_{2}\right)$ leva $x\left(t_{2}\right)$ em $x_{2} \stackrel{\text { def }}{=} A\left(t_{2}\right) x\left(t_{2}\right)$ e assim por diante.

O sistema impulsivo descrito acima é denotado por

$$
\begin{gathered}
\dot{x}=f(t, x) \\
x(t) \in M(t) \Rightarrow x\left(t^{+}\right)=A(t) x(t) .
\end{gathered}
$$

Neste trabalho, além de nos dedicarmos a sistemas impulsivos com retardamento, vamos nos ater ao importante caso onde $M(t)=M$ e $A(t)=A: M \rightarrow M$ não dependem de $t \in \mathbb{R}_{+}$e a equação é autônoma. Esta situação, segundo Myshkis [7], é chamada de sistema impulsivo com condição de auto-sustentação.

Para as equações com retardamento as condições de impulso trazem algumas dificuldades que não se apresentam no caso ordinário. 
O ponto é que os impulsos representam, em geral, descontinuidades de primeira espécie dos termos $x_{t}$ pertencentes ao espaço de fase.

Assim, o espaço de fase clássico, $C\left([-r, 0], \mathbb{R}^{n}\right)$, já não serve mais. Por esse motivo obtemos no Capítulo 1 alguns resultados básicos de uma teoria geral em $L^{p}$ das equações diferenciais funcionais com retardamento.

Embora este capítulo vise atender os propósitos do Capítulo 3, os resultados ali estabelecidos estão num contexto mais geral do que o necessário.

O Capítulo 2 inicia-se com alguns resultados de Nussbaum [9] que compõem o contexto deste trabalho. Depois desenvolve-se um estudo de uma equação planar que é uma variante da equação estudada no Capítulo 3. É estabelecida uma seqüência de bifurcações de Hopf.

No Capítulo 3 obtemos um teorema de existência de soluções periódicas lentamente espiralantes para uma classe de sistemas impulsivos com retardamento. Esse teorema é estabelecido por uma combinação da condição de impulso com a ejetividade da origem em relação a um operador de retorno. 


\section{Capítulo 1}

\section{Fatos básicos de uma teoria geral $\operatorname{em} L^{p}$}

\subsection{Introdução}

Neste capítulo vamos introduzir fatos básicos de uma teoria geral para equações diferenciais com retardamento tomando um espaço $L^{p}$ como espaço de fase. Não temos a intenção de construir uma teoria completa, mas pretendemos desenvolver uma parte dela que venha suprir nossas necessidades no Capítulo 3. Essas necessidades têm origem na ocorrência de impulsos, os quais introduzem descontinuidades nas soluções, tornando inadequado o espaço de fase clássico $C\left([-r, 0], \mathbb{R}^{n}\right)$. Uma das diferenças essenciais na construção dessa teoria é que as condições iniciais $\varphi \in L^{p}$ não determinam o valor inicial da solução. Os resultados deste capítulo, bem como as notações, têm forte inspiração na teoria clássica segundo Hale, veja [5].

\subsection{Definições preliminares}

Seja $x$ uma função definida q.t.p. em $[\sigma-r, \sigma)$ e contínua em $[\sigma, \sigma+\alpha)$, para algum $\alpha>0$. Para cada $t \in[\sigma, \sigma+\alpha)$, definimos ${ }^{t} x=\left(x_{t}, x(t)\right)$ sendo $x_{t}(\cdot)=x(t+\cdot)$ q.t.p. 
em $(-r, 0)$.

Consideremos o espaço

$$
L^{p}=L^{p}((-r, 0), X)=\left\{\left.\varphi\left|\int_{(-r, 0)}\right| \varphi\right|^{p}<\infty\right\}
$$

com $\|\varphi\|_{p}=\left(\int_{(-r, 0)}|\varphi|^{p}\right)^{1 / p}$, e a equação diferencial funcional com retardamento

$$
\dot{x}(t)=F\left(t,{ }^{t} x\right),
$$

onde $X=\mathbb{R}^{n}, 1 \leq p<\infty, F: \Omega \rightarrow X, \Omega$ um subconjunto aberto de $\mathbb{R} \times L^{p} \times X$.

Definição 1.1 A função $x$ é dita solução da equação (1.1) se existe $\alpha>0$ tal que $x \in L_{l o c}^{p}((\sigma-r, \sigma+\alpha), X), x$ tem um representante tal que $\left.x\right|_{[\sigma, \sigma+\alpha)}$ é absolutamente contínuo, $\left(t,{ }^{t} x\right) \in \Omega$ para todo $t \in[\sigma, \sigma+\alpha)$ e $\dot{x}(t)=F\left(t,{ }^{t} x\right)$ q.t.p. em $[\sigma, \sigma+\alpha)$.

Dados $\sigma \in \mathbb{R}, \varphi \in L^{p}$ e $\xi \in X$ consideremos o problema de valor inicial (P.V.I)

$$
\left\{\begin{array}{l}
\dot{x}(t)=F\left(t,{ }^{t} x\right) \\
{ }^{\sigma} x=(\varphi, \xi) .
\end{array}\right.
$$

Definição 1.2 Dizemos que $x=x(\sigma, \varphi, \xi, F)$ é uma solução do problema de valor inicial (1.2) se existe $\alpha>0$ tal que $x$ é um elemento de $L_{l o c}^{p}((\sigma-r, \sigma+\alpha), X), x_{\sigma}=\varphi$ q.t.p., $x(\sigma)=\xi$, $x$ tem um representante absolutamente continuo em $[\sigma, \sigma+\alpha)$, $\left(t,{ }^{t} x\right) \in \Omega$ para todo $t \in[\sigma, \sigma+\alpha)$ e $\dot{x}(t)=F\left(t,{ }^{t} x\right)$ q.t.p. em $[\sigma, \sigma+\alpha)$.

\subsection{Existência}

Vamos dar um teorema de existência de soluções para o P.V.I acima assumindo que $F$ seja contínua. Para provar a existência de solução passando por $(\sigma, \varphi, \xi) \in \mathbb{R} \times L^{p} \times X$, consideramos um $\alpha>0$ e todas as funções $x$ em $L_{l o c}^{p}((\sigma-r, \sigma+\alpha), X)$, que coincidem $\operatorname{com} \varphi$ q.t.p. em $(\sigma-r, \sigma)$ e $x(\sigma)=\xi$. Utilizaremos a equação integral correspondente 
a (1.2) para definir um operador $T$ e provaremos o teorema pela existência de ponto fixo de $T$ via Teorema de Schauder.

Apresentamos agora alguns lemas auxiliares e notações que serão úteis a seguir.

O papel do Teorema de Ascoli-Arzela, que funciona como critério de compacidade na teoria clássica, é aqui desempenhado pelo Teorema de Fréchet-Kolmogorov.

Lema 1.1 Seja $x \in L_{l o c}^{p}((\sigma-r, \sigma+\alpha), X)$ tal que $F\left(t,{ }^{t} x\right)$ é localmente integrável em $[\sigma, \sigma+\alpha)$. Então x é solução de (1.1) passando por $(\sigma, \varphi, \xi)$ se, e somente se,

$$
\left\{\begin{array}{l}
x(t)=\xi+\int_{\sigma}^{t} F\left(s,{ }^{s} x\right) d s, \quad \text { se } t \in[\sigma, \sigma+\alpha) \\
x_{\sigma}=\varphi \quad \text { q.t.p }
\end{array}\right.
$$

Prova A prova segue diretamente do teorema fundamental do cálculo.

Observação 1.1 Em particular, se F satisfaz as condições de Caratheodory ou é contínua, então a hipótese do lema acima de que $F\left(t,{ }^{t} x\right)$ é localmente integrável está satisfeita.

Lema 1.2 Se $x \in L_{l o c}^{p}((\sigma-r, \sigma+\alpha), X)$, então a aplicação $t \rightarrow x_{t} \in L^{p}$ é contínua.

Prova Observemos que $x \in L^{p}\left(\left(\sigma-r, \sigma+\alpha^{\prime}\right), X\right)$, para todo $\alpha^{\prime}<\alpha$.

Assim, para cada $\alpha^{\prime}, 0<\alpha^{\prime}<\alpha$, dado $\varepsilon>0$, existe $\delta>0$ tal que $|h|<\delta$ implica $\int_{\sigma-r}^{\sigma+\alpha^{\prime}}|x(u+h)-x(u)|^{p} d u<\varepsilon^{p}(\operatorname{definimos} x(t)=0$, se $t<\sigma-r)$. Fixado $t \in\left[\sigma, \sigma+\alpha^{\prime}\right)$

$$
\int_{t-r}^{t}|x(u+h)-x(u)|^{p} d u<\int_{\sigma-r}^{\sigma+\alpha^{\prime}}|x(u+h)-x(u)|^{p} d u<\varepsilon^{p} .
$$

Façamos a mudança de variável $u=t+\theta$. Assim,

$$
\int_{t-r}^{t}|x(u+h)-x(u)|^{p} d u=\int_{-r}^{0}|x(t+\theta+h)-x(t+\theta)|^{p} d \theta<\varepsilon^{p} .
$$


Consideremos $\tau$ de modo que $|\tau-t|=|h|<\delta$.

$$
\int_{-r}^{0}|x(\tau+\theta)-x(t+\theta)|^{p} d \theta=\int_{-r}^{0}\left|x_{\tau}(\theta)-x_{t}(\theta)\right|^{p} d \theta<\varepsilon^{p}
$$

ou seja, $\left\|x_{\tau}-x_{t}\right\|_{p}<\varepsilon$.

Definição 1.3 Seja $(\sigma, \varphi, \xi) \in \mathbb{R} \times L^{p} \times X$. Definimos $\widetilde{\varphi} \in L^{p}((\sigma-r, \sigma+\alpha), X)$ por

$$
\left\{\begin{array}{l}
\widetilde{\varphi}_{\sigma}=\varphi \quad \text { q.t.p. } \\
\widetilde{\varphi}(t+\sigma)=\xi, \quad \text { se } t \in[0, \alpha)
\end{array}\right.
$$

Lema 1.3 Seja $x(t+\sigma)=\widetilde{\varphi}(t+\sigma)+y(t)$ q.t.p. se $t \in(-r, 0)$ e $x(t+\sigma)=\widetilde{\varphi}(t+\sigma)+y(t)$ para todo $t \in[0, \alpha)$. Então $x$ é uma solução de

$$
\left\{\begin{array}{l}
x_{\sigma}=\varphi \quad \text { q.t.p. } \\
x(t)=\xi+\int_{\sigma}^{t} F\left(s,{ }^{s} x\right) d s, \quad \text { se } t \in[\sigma, \sigma+\alpha)
\end{array}\right.
$$

se, e somente se, y é uma solução de

$$
\left\{\begin{array}{l}
y_{0}=0 \quad \text { q.t.p. } \\
y(t)=\int_{0}^{t} F\left(s+\sigma, \widetilde{\varphi}_{s+\sigma}+y_{s}, \widetilde{\varphi}(s+\sigma)+y(s)\right) d s, \quad \text { se } t \in[0, \alpha) .
\end{array}\right.
$$

Prova Seja $x$ solução de (1.5). Seja $y(t)$ dado no enunciado do Lema 1.3. Então $y(t)=x(t+\sigma)-\widetilde{\varphi}(t+\sigma)=x_{\sigma}(t)-\widetilde{\varphi}_{\sigma}(t)=\varphi(t)-\varphi(t)=0$ q.t.p. se $t \in(-r, 0)$, $y(0)=x(\sigma)-\widetilde{\varphi}(\sigma)=\xi-\xi=0 \mathrm{e}$ $y(t)=x(t+\sigma)-\widetilde{\varphi}(t+\sigma)=\int_{\sigma}^{t+\sigma} F\left(s,{ }^{s} x\right) d s$, se $t \in[0, \alpha)$.

Fazendo uma mudança de variáveis obtemos

$$
y(t)=\int_{0}^{t} F\left(s+\sigma, x_{s+\sigma}, x(s+\sigma)\right) d s=\int_{0}^{t} F\left(s+\sigma, \widetilde{\varphi}_{s+\sigma}+y_{s}, \widetilde{\varphi}(s+\sigma)+y(s)\right) d s,
$$

se $t \in[0, \alpha)$. 
Reciprocamente, sejam $y$ solução de (1.6) e $x$ como no enunciado do Lema 1.3. Então, $x(t+\sigma)=\widetilde{\varphi}(t+\sigma)+y(t)=\varphi(t)-0=\varphi(t)$ q.t.p. se $t \in(-r, 0)$, $x(\sigma)=\widetilde{\varphi}(\sigma)+y(0)=\xi+0=\xi \mathrm{e}$

$$
\begin{aligned}
x(t+\sigma)=\widetilde{\varphi}(t+\sigma)+\int_{0}^{t} F\left(s+\sigma, \widetilde{\varphi}_{s+\sigma}+y_{s}, \widetilde{\varphi}(s+\sigma)+y(s)\right) d s= & \\
& =\xi+\int_{\sigma}^{t+\sigma} F\left(s,{ }^{s} x\right) d s, \text { se } t \in[0, \alpha),
\end{aligned}
$$

ou seja, $x(t)=\xi+\int_{\sigma}^{t} F\left(s,{ }^{s} x\right) d s$, se $t \in[\sigma, \sigma+\alpha)$.

Sendo assim, encontrar uma solução $x$ de (1.5) é equivalente a encontrar $\alpha>0$ e $y \in L_{l o c}^{p}((-r, \alpha), X)$ tal que $y$ satisfaça $(1.6)$.

Definição 1.4 Seja $V$ um subconjunto de $\mathbb{R} \times L^{p} \times X$. Denotemos por $C(V, X)$ o conjunto das funções contínuas $F: V \rightarrow X$ e por $C^{0}(V, X)$ o conjunto das funções $F \in C(V, X)$ limitadas. Para $F \in C^{0}(V, X)$, definimos $|F|=|F|_{V}=\sup _{x \in V}|F(x)|$.

Definição 1.5 Para quaisquer reais $\alpha, \beta, \gamma>0$ definimos

$I_{\alpha}=[0, \alpha)$,

$B_{\beta}=\left\{\psi \in L^{p} \mid\|\psi\|_{p} \leq \beta\right\}$,

$D_{\gamma}=\{\xi \in X|| \xi \mid \leq \gamma\} e$

$\mathcal{A}(\alpha, \beta, \gamma)=\left\{y \in L^{p}((-r, \alpha), X) \mid y\right.$ possui um representante absolutamente

contínuo em $I_{\alpha}, 0 \in y_{0} \in L^{p}, y(0)=0, y_{t} \in B_{\beta}$ e $y(t) \in D_{\gamma}$, para todo $\left.t \in I_{\alpha}\right\}$.

Lema 1.4 Suponhamos dados um aberto $\Omega$ em $\mathbb{R} \times L^{p} \times X$, um compacto $W \subset \Omega$ e $F^{0} \in C(\Omega, X)$. Então

a) existe uma vizinhança $V \subset \Omega$ de $W$ tal que $F^{0} \in C^{0}(V, X)$,

b) existem uma vizinhança $U \subset C^{0}(V, X)$ de $F^{0}$ e constante positiva $M$ tais que $|F(\sigma, \varphi, \xi)|<M$ para todo $(\sigma, \varphi, \xi) \in V$ e toda $F \in U$

c) existem constantes positivas $\alpha, \beta$ e $\gamma$ tais que para qualquer $\left(\sigma^{0}, \varphi^{0}, \xi^{0}\right) \in W$ temos $\left(\sigma^{0}+t, \widetilde{\varphi}_{\sigma^{0}+t}^{0}+y_{t}, \widetilde{\varphi}^{0}\left(\sigma^{0}+t\right)+y(t)\right) \in V$ para todo $t \in I_{\alpha}$ e $y \in \mathcal{A}(\alpha, \beta, \gamma)$. 
Prova a) Como $W$ é compacto e $F^{0}$ é contínua em $\Omega$, existe uma constante $M>0$ tal que $\left|F^{0}\left(\sigma^{0}, \varphi^{0}, \xi^{0}\right)\right|<M$, para todo $\left(\sigma^{0}, \varphi^{0}, \xi^{0}\right) \in W$.

Além disso, existem constantes positivas $\bar{\alpha}, \bar{\beta}, \bar{\gamma}$ e $\varepsilon$ tais que

$$
\left|F^{0}\left(\sigma^{0}+t, \varphi^{0}+\varphi, \xi^{0}+\xi\right)\right|<M-\varepsilon, \forall\left(\sigma^{0}, \varphi^{0}, \xi^{0}\right) \in W \text { e }(t, \varphi, \xi) \in I_{\bar{\alpha}} \times B_{\bar{\beta}} \times D_{\bar{\gamma}}
$$

De fato. Se isto não ocorre, então para quaisquer $\bar{\alpha}, \bar{\beta}, \bar{\gamma} \mathrm{e} \varepsilon$, existem $\left(\sigma_{\varepsilon}^{0}, \varphi_{\varepsilon}^{0}, \xi_{\varepsilon}^{0}\right) \in W$ e $\left(t_{\bar{\alpha}}, \varphi_{\bar{\beta}}, \xi_{\bar{\gamma}}\right) \in I_{\bar{\alpha}} \times B_{\bar{\beta}} \times D_{\bar{\gamma}}$ tais que

$$
\left|F^{0}\left(\sigma_{\varepsilon}^{0}+t_{\bar{\alpha}}, \varphi_{\varepsilon}^{0}+\varphi_{\bar{\beta}}, \xi_{\varepsilon}^{0}+\xi_{\bar{\gamma}}\right)\right| \geq M-\varepsilon .
$$

Logo, tomando-se seqüências $\bar{\alpha}_{n}, \bar{\beta}_{n}, \bar{\gamma}_{n}$ e $\varepsilon_{n}$ convergindo para zero, existem $\left(\sigma_{\varepsilon_{n}}^{0}, \varphi_{\varepsilon_{n}}^{0}, \xi_{\varepsilon_{n}}^{0}\right) \in W$ e $\left(t_{\bar{\alpha}_{n}}, \varphi_{\bar{\beta}_{n}}, \xi_{\overline{\gamma_{n}}}\right) \in I_{\bar{\alpha}_{n}} \times B_{\bar{\beta}_{n}} \times D_{\overline{\gamma_{n}}}$ de modo que

$$
\left|F^{0}\left(\sigma_{\varepsilon_{n}}^{0}+t_{\bar{\alpha}_{n}}, \varphi_{\varepsilon_{n}}^{0}+\varphi_{\bar{\beta}_{n}}, \xi_{\varepsilon_{n}}^{0}-\xi_{\bar{\gamma}_{n}}\right)\right| \geq M-\varepsilon_{n}
$$

Como $W$ é compacto, existe $\left(\sigma_{0}^{0}, \varphi_{0}^{0}, \xi_{0}^{0}\right) \in W$ tal que $\left(\sigma_{\varepsilon_{n}}^{0}, \varphi_{\varepsilon_{n}}^{0}, \xi_{\varepsilon_{n}}^{0}\right) \rightarrow\left(\sigma_{0}^{0}, \varphi_{0}^{0}, \xi_{0}^{0}\right)$ em $\mathbb{R} \times L^{p} \times X$ (se necessário passe a uma subseqüência). Assim

$$
\lim _{n \rightarrow \infty}\left|F^{0}\left(\sigma_{\varepsilon_{n}}^{0}+t_{\bar{\alpha}_{n}}, \varphi_{\varepsilon_{n}}^{0}+\varphi_{\overline{\beta_{n}}}, \xi_{\varepsilon_{n}}^{0}+\xi_{\overline{\gamma_{n}}}\right)\right| \geq \lim _{n \rightarrow \infty}\left(M-\varepsilon_{n}\right),
$$

ou seja,

$$
\left|F^{0}\left(\lim _{n \rightarrow \infty}\left(\sigma_{\varepsilon_{n}}^{0}+t_{\bar{\alpha}_{n}}\right), \lim _{n \rightarrow \infty}\left(\varphi_{\varepsilon_{n}}^{0}+\varphi_{\overline{\beta_{n}}}\right), \lim _{n \rightarrow \infty}\left(\xi_{\varepsilon_{n}}^{0}+\xi_{\overline{\gamma_{n}}}\right)\right)\right| \geq M .
$$

Sabemos que,

$$
\begin{gathered}
\lim _{n \rightarrow \infty} \bar{\alpha}_{n}=0 \Longrightarrow \lim _{n \rightarrow \infty} t_{\bar{\alpha}_{n}}=0, \\
\lim _{n \rightarrow \infty} \bar{\beta}_{n}=0 \Longrightarrow \lim _{n \rightarrow \infty} \varphi_{\overline{\beta_{n}}}=0 \text { q.t.p } \\
\text { e } \lim _{n \rightarrow \infty} \bar{\gamma}_{n}=0 \Longrightarrow \lim _{n \rightarrow \infty} \xi_{\overline{\gamma_{n}}}=0 .
\end{gathered}
$$

Assim, $\left|F^{0}\left(\sigma_{0}^{0}, \varphi_{0}^{0}, \xi_{0}^{0}\right)\right| \geq M$, o que é uma contradição.

Deste modo, se definirmos

$$
V=\left\{\left(\sigma^{0}+t, \varphi^{0}+\varphi, \xi^{0}+\xi\right) \mid\left(\sigma^{0}, \varphi^{0}, \xi^{0}\right) \in W,(t, \varphi, \xi) \in I_{\bar{\alpha}} \times B_{\bar{\beta}} \times D_{\bar{\gamma}}\right\}
$$


teremos $V \subset \Omega$, para $\bar{\alpha}, \bar{\beta}$ e $\bar{\gamma}$ suficientemente pequenos, e $F^{0}$ limitada em $V$.

b) Para $\varepsilon>0$ do item a), seja $U=\left\{F \in C(V, X)|| F-\left.F^{0}\right|_{V}<\varepsilon\right\}$. Então se $F \in U$ e $(\sigma, \varphi, \xi) \in V$ temos

$$
\begin{aligned}
& |F(\sigma, \varphi, \xi)|=\left|F(\sigma, \varphi, \xi)-F^{0}(\sigma, \varphi, \xi)+F^{0}(\sigma, \varphi, \xi)\right| \leq \\
& \quad \leq\left|F(\sigma, \varphi, \xi)-F^{0}(\sigma, \varphi, \xi)\right|+\left|F^{0}(\sigma, \varphi, \xi)\right| \leq\left|F-F^{0}\right|_{V}+M-\varepsilon<M .
\end{aligned}
$$

Portanto, $|F(\sigma, \varphi, \xi)|<M$, para todo $(\sigma, \varphi, \xi) \in V$ e $U \subset C^{0}(V, X)$.

c) Seja $0<\beta<\bar{\beta}$ e $0<\gamma<\bar{\gamma}$. Escolhamos $\alpha$ tal que

$$
0<\alpha<\bar{\alpha}, \quad\left\|\widetilde{\varphi}_{\sigma^{0}+t}^{0}-\varphi^{0}\right\|_{p}<\bar{\beta}-\beta, \quad\left|\widetilde{\varphi}^{0}\left(\sigma^{0}+t\right)-\xi^{0}\right|<\bar{\gamma}-\gamma
$$

para todo $\left(\sigma^{0}, \varphi^{0}, \xi^{0}\right) \in W$ e $t \in I_{\alpha}$, com $\bar{\alpha}, \bar{\beta}$ e $\bar{\gamma}$ do item a).

De fato. Suponhamos que esta escolha não fosse possível, ou seja, para cada $\alpha<\bar{\alpha}$ $\operatorname{existam}\left(\sigma_{\alpha}^{0}, \varphi_{\alpha}^{0}, \xi_{\alpha}^{0}\right) \in W$ e $t_{\alpha} \in I_{\alpha}$ tais que

$$
\left\|\widetilde{\varphi}_{\alpha_{\alpha}^{0}+t_{\alpha}}^{0}-\varphi_{\alpha}^{0}\right\|_{p} \geq \bar{\beta}-\beta \quad \text { ou } \quad\left|\widetilde{\varphi}_{\alpha}^{0}\left(\sigma_{\alpha}^{0}+t_{\alpha}\right)-\xi_{\alpha}^{0}\right| \geq \bar{\gamma}-\gamma
$$

Em particular, existem seqüências $\alpha_{n} \rightarrow 0$ e $\left(\sigma_{\alpha_{n}}^{0}, \varphi_{\alpha_{n}}^{0}, \xi_{\alpha_{n}}^{0}\right) \in W$ tais que

$$
\left\|\widetilde{\varphi}_{\alpha_{n} \sigma_{\alpha_{n}}^{0}+t_{\alpha_{n}}}^{0}-\varphi_{\alpha_{n}}^{0}\right\|_{p} \geq \bar{\beta}-\beta \quad \text { ou } \quad\left|\widetilde{\varphi}_{\alpha_{n}}^{0}\left(\sigma_{\alpha_{n}}^{0}+t_{\alpha_{n}}\right)-\xi_{\alpha_{n}}^{0}\right| \geq \bar{\gamma}-\gamma
$$

Como $\left(\sigma_{\alpha_{n}}^{0}, \varphi_{\alpha_{n}}^{0}, \xi_{\alpha_{n}}^{0}\right) \in W$ que é compacto, podemos supor $\left(\sigma_{\alpha_{n}}^{0}, \varphi_{\alpha_{n}}^{0}, \xi_{\alpha_{n}}^{0}\right)$ convergente para, digamos, $\left(\sigma_{0}^{0}, \varphi_{0}^{0}, \xi_{0}^{0}\right) \in W$. Também como a função $t \rightarrow x_{t} \in L^{p}$ é contínua e usando o Teorema da Convergência Dominada de Lebesgue temos

$$
\lim _{n \rightarrow \infty}\left\|\widetilde{\varphi}_{\alpha_{n} \sigma_{\alpha_{n}+\alpha_{n}}^{0}}^{0}-\varphi_{\alpha_{n}}^{0}\right\|_{p}^{p} \geq(\bar{\beta}-\beta)^{p} \text {, isto é, } \lim _{n \rightarrow \infty} \int\left|\widetilde{\varphi}_{\alpha_{n_{\sigma_{\alpha}}}^{0}+t_{\alpha_{n}}}^{0}-\varphi_{\alpha_{n}}^{0}\right|^{p} \geq(\bar{\beta}-\beta)^{p}
$$

o que implica

$$
\int \lim _{n \rightarrow \infty}\left|\widetilde{\varphi}_{\alpha_{\sigma_{\sigma_{n}}+t_{\alpha_{n}}}^{0}}^{0}-\varphi_{\alpha_{n}}^{0}\right|^{p} \geq(\bar{\beta}-\beta)^{p} \Longrightarrow \int\left|\varphi_{\alpha_{n}}^{0} \operatorname{sigma}^{0}-\varphi_{\alpha_{n}}^{0}\right|^{p} \geq(\bar{\beta}-\beta)^{p}
$$


ou seja, $\bar{\beta} \leq \beta$, que é uma contradição.

Se $\left|\widetilde{\varphi}_{\alpha_{n}}^{0}\left(\sigma_{\alpha_{n}}^{0}+t_{\alpha_{n}}\right)-\xi_{\alpha_{n}}^{0}\right| \geq \bar{\gamma}-\gamma$ então $\lim _{n \rightarrow \infty}\left|\widetilde{\varphi}_{\alpha_{n}}^{0}\left(\sigma_{\alpha_{n}}^{0}+t_{\alpha_{n}}\right)-\xi_{\alpha_{n}}^{0}\right| \geq \bar{\gamma}-\gamma$. Logo, $\left|\lim _{n \rightarrow \infty}\left(\widetilde{\varphi}_{\alpha_{n}}^{0}\left(\sigma_{\alpha_{n}}^{0}+t_{\alpha_{n}}\right)-\xi_{\alpha_{n}}^{0}\right)\right| \geq \bar{\gamma}-\gamma$ o que nos leva à contradição $\bar{\gamma} \leq \gamma$.

Sendo assim, da maneira que $V$ foi construída, a prova está completa, pois para quaisquer $\left(\sigma^{0}, \varphi^{0}, \xi^{0}\right) \in W, t \in I_{\alpha}$ e $y \in \mathcal{A}(\alpha, \beta, \gamma)$, temos

$$
\begin{aligned}
& \left(\sigma^{0}+t, y_{t}+\widetilde{\varphi}_{t+\sigma^{0}}^{0}, y(t)+\widetilde{\varphi}^{0}\left(t+\sigma^{0}\right)\right)= \\
& \quad=\left(\sigma^{0}+t, \varphi^{0}+\left(y_{t}+\widetilde{\varphi}_{t+\sigma^{0}}^{0}-\varphi^{0}\right), \xi^{0}+\left(y(t)+\widetilde{\varphi}^{0}\left(t+\sigma^{0}\right)-\xi^{0}\right)\right) \in V
\end{aligned}
$$

pois

$$
\begin{gathered}
\left\|y_{t}+\widetilde{\varphi}_{t+\sigma^{0}}^{0}-\varphi^{0}\right\|_{p} \leq\left\|y_{t}\right\|_{p}+\left\|\widetilde{\varphi}_{t+\sigma^{0}}^{0}-\varphi^{0}\right\|_{p}<\beta+(\bar{\beta}-\beta)=\bar{\beta} \quad \text { e } \\
\left|y(t)+\widetilde{\varphi}^{0}\left(\sigma^{0}+t\right)-\xi^{0}\right| \leq|y(t)|+\left|\widetilde{\varphi}^{0}\left(\sigma^{0}+t\right)-\xi^{0}\right|<\gamma+(\bar{\gamma}-\gamma)=\bar{\gamma} .
\end{gathered}
$$

O Lema 1.5 a seguir estabelece que um certo operador integral é completamente contínuo e será fundamental na prova de existência de soluções de (1.6) através do Teorema de Schauder. Essa estratégia depende do Teorema de Fréchet-Kolmogorov, veja [3], que enunciamos agora. Para isso precisaremos das seguintes definições.

Definição 1.6 Seja $\Xi \subset \mathbb{R}^{n}$ aberto. Dizemos que o conjunto $\omega$ está fortemente contido em $\Xi$ se $\bar{\omega} \subset \Xi e \bar{\omega}$ é compacto. Neste caso, usamos a seguinte notação $\omega \subset \subset \Xi$.

Definição 1.7 Definiremos $\tau_{h} g$ por $\left(\tau_{h} g\right)(t)=g(t+h)$.

Teorema 1.1 (Fréchet-Kolmogorov) Seja $\Xi \subset \mathbb{R}^{n}$ um aberto e $\mathcal{F}$ um subconjunto limitado de $L^{p}(\Xi)$ com $1 \leq p<\infty$. Suponhamos que

(i) para todo $\varepsilon>0$ e todo $\omega \subset \subset \Xi$, existe um $\delta>0, \delta<\operatorname{dist}\left(\omega, \Xi^{c}\right)$ tal que

$$
\left\|\tau_{h} g-g\right\|_{L^{p}(\omega)}<\varepsilon \text {, para todo } h \in \mathbb{R}^{n} \operatorname{com}|h|<\delta \text { e toda } g \in \mathcal{F}
$$


(ii) para todo $\varepsilon>0$ existe $\omega \subset \subset \Xi$ tal que $\|g\|_{L^{p}(\Xi \backslash \omega)}<\varepsilon$, para toda $g \in \mathcal{F}$.

Então $\mathcal{F}$ é relativamente compacto em $L^{p}(\Xi)$.

Lema 1.5 Sejam dados um aberto $\Omega \subset \mathbb{R} \times L^{p} \times X$, um compacto $W \subset \Omega$ e uma função $F^{0} \in C(\Omega, X)$. Consideremos as vizinhanças $U, V$ e as constantes $M, \alpha, \beta, \gamma$, obtidas no Lema 1.4 de modo que $\alpha \leq \min \left\{\left[\beta(p+1)^{1 / p} / M\right]^{p / p+1}, \gamma / M\right\}$. Seja $T: W \times U \times \mathcal{A}(\alpha, \beta, \gamma) \rightarrow L^{p}((-r, \alpha), X)$ onde $(\sigma, \varphi, \xi, F, y) \rightarrow T(\sigma, \varphi, \xi, F, y) e$

$$
T(\sigma, \varphi, \xi, F, y)(t)= \begin{cases}0 & , \text { q.t.pem }(-r, 0) \\ \int_{0}^{t} F\left(\sigma+s, \widetilde{\varphi}_{\sigma+s}+y_{s}, \widetilde{\varphi}(\sigma+s)+y(s)\right) d s & , \text { se } t \in I_{\alpha}\end{cases}
$$

\section{Então}

a) existe um compacto $K$ em $L^{p}((-r, \alpha), X)$ tal que $T: W \times U \times \mathcal{A}(\alpha, \beta, \gamma) \rightarrow K$,

b) se $\alpha \leq \min \left\{\left[\beta(p+1)^{1 / p} / M\right]^{p / p+1}, \gamma / M\right\}$ então

$$
T: W \times U \times \mathcal{A}(\alpha, \beta, \gamma) \rightarrow \mathcal{A}(\alpha, \beta, \gamma)
$$

c) e $T: W \times U \times \mathcal{A}(\alpha, \beta, \gamma) \rightarrow L^{p}((-r, \alpha), X)$ é contínua.

Prova É claro que $T(\sigma, \varphi, \xi, F, y)$ é um elemento de $L^{p}((-r, \alpha), X)$. Usaremos a notação $\|\cdot\|_{L^{p}}$ para a norma de um elemento de $L^{p}((-r, \alpha), X)$.

a) Pelo Lema 1.4 existem uma vizinhança $U \subset C^{0}(V, X)$ de $F^{0}$ e constante positiva $M$ tais que $|F(\sigma, \varphi, \xi)|<M$, para todo $(\sigma, \varphi, \xi) \in V$ e $F \in U$.

Sejam $\rho, 0<\rho<\min (r, \alpha)$, e $\mathcal{F}(\rho) \subset L^{p}((-r, \alpha), X)$ de modo que $g \in \mathcal{F}(\rho)$ se, e somente se, as seguintes condições estão satisfeitas

(i) $g$ é absolutamente contínua em $I_{\alpha}, g=0$ q.t.p. em $(-r, 0)$ e $|g(t)| \leq M \alpha$ para todo $t \in I_{\alpha}$, 
(ii) $\|g\|_{L^{p}} \leq M\left(\alpha^{p+1} /(p+1)\right)^{1 / p}$,

(iii) $\left\|\tau_{h} g-g\right\|_{L^{p}((-r+\rho, \alpha-\rho), X)} \leq M|h|(\alpha+r-2 \rho)^{1 / p}$,

(iv) $\|g\|_{L^{p}((-r, \alpha) \backslash(-r+\rho, \alpha-\rho), X)} \leq M\left[\left(\alpha^{p+1}-(\alpha-\rho)^{p+1}\right) /(p+1)\right]^{1 / p}$.

Afirmação: Existe $\bar{\rho}$ tal que $\mathcal{F}(\bar{\rho})$ é relativamente compacto.

De fato.

Observemos que, por (ii), $\mathcal{F}(\rho)$ é limitado em $L^{p}((-r, \alpha), X)$.

Dado $\varepsilon>0$, seja $\varepsilon^{\prime}=\left((p+1)^{1 / p} / M\right) \varepsilon$. Então existe $\delta_{1}>0$ tal que

$$
\alpha^{p+1}-(\alpha-\rho)^{p+1}<\left(\varepsilon^{\prime}\right)^{p}
$$

para todo $\rho<\delta_{1}$ (isto é possível pois a função $z \rightarrow z^{p+1}$ é contínua). Assim, para $0<\rho<\delta_{1}$ temos $\|g\|_{L^{p}((-r, \alpha) \backslash(-r+\rho, \alpha-\rho), X)}<\varepsilon$.

Seja $w_{1} \subset \subset(-r, \alpha)$ e $\rho_{2}>0$ tal que $w_{1} \subset\left(-r+\rho_{2}, \alpha-\rho_{2}\right)$. Dado $\varepsilon>0$, seja $\delta_{2}<\min \left\{\rho_{2}, \varepsilon / M\left(\alpha+r-2 \rho_{2}\right)^{1 / p}\right\}$. Então,

$$
\left\|\tau_{h} g-g\right\|_{L^{p}\left(w_{1}\right)}<\left\|\tau_{h} g-g\right\|_{L^{p}\left(\left(-r+\rho_{2}, \alpha-\rho_{2}\right), X\right)} \leq M|h|\left(\alpha+r-2 \rho_{2}\right)^{1 / p}<\varepsilon,
$$

para todo $|h|<\delta_{2}$ e toda $g \in \mathcal{F}\left(\rho_{2}\right)$. Logo, existe $\bar{\rho}$, tal que $\mathcal{F}(\bar{\rho})$ é relativamente compacto. Portanto, $K:=\overline{\mathcal{F}(\bar{\rho})}$ é compacto.

Seja $\rho<\bar{\rho}$.

Denotemos abreviadamente $T(y)=T(\sigma, \varphi, \xi, F, y)$ e observemos que:

- se $t \in(-r+\rho, 0)$ e $t+h \in(-r, 0)$, então $|T(y)(t+h)-T(y)(t)|^{p}=0$ q.t.p, o que implica

$$
\left\|\tau_{h} T(y)-T(y)\right\|_{L^{p}((-r+\rho, \alpha-\rho), X)}^{p}=0 \leq M^{p}|h|^{p}(\alpha+r-2 \rho)
$$

- se $t \in(-r+\rho, 0)$ e $t+h \in[0, \alpha)$, então $|T(y)(t+h)-T(y)(t)|^{p}=|T(y)(t+h)|^{p} \leq$ 
$\leq\left(\int_{0}^{t+h}\left|F\left(\sigma+s, \widetilde{\varphi}_{\sigma+s}+y_{s}, \widetilde{\varphi}(\sigma+s)+y(s)\right)\right| d s\right)^{p} \leq M^{p}(t+h)^{p} \leq M^{p}|h|^{p}, \mathrm{o}$ que implica

$$
\left\|\tau_{h} T(y)-T(y)\right\|_{L^{p}((-r+\rho, \alpha-\rho), X)}^{p} \leq \int_{-r+\rho}^{\alpha-\rho} M^{p}|h|^{p} d t \leq M^{p}|h|^{p}(\alpha+r-2 \rho)
$$

- se $t \in[0, \alpha-\rho)$ e $t+h \in(-r, 0)$, então $h<0$. Consideremos $t+h=u$. Assim,

$$
\begin{aligned}
& t=u+|h| \text { e }|T(y)(t+h)-T(y)(t)|^{p}=|T(y)(u)-T(y)(u+|h|)|^{p}= \\
& =|T(y)(u+|h|)|^{p}=\left|\int_{0}^{u+|h|} F\left(\sigma+s, \widetilde{\varphi}_{\sigma+s}+y_{s}, \widetilde{\varphi}(\sigma+s)+y(s)\right) d s\right|^{p} \leq \\
& \leq\left(\int_{u}^{u+|h|}\left|F\left(\sigma+s, \widetilde{\varphi}_{\sigma+s}+y_{s}, \widetilde{\varphi}(\sigma+s)+y(s)\right)\right| d s\right)^{p} \leq M^{p}|h|^{p}, \text { o que implica } \\
& \quad\left\|\tau_{h} T(y)-T(y)\right\|_{L^{p}((-r+\rho, \alpha-\rho), X)}^{p} \leq \int_{-r+\rho}^{\alpha-\rho} M^{p}|h|^{p} d t \leq M^{p}|h|^{p}(\alpha+r-2 \rho)
\end{aligned}
$$

- se $t \in[0, \alpha-\rho)$ e $t+h \in[0, \alpha)$, então

$$
\begin{aligned}
& |T(y)(t+h)-T(y)(t)|^{p}=\left|\int_{t}^{t+h} F\left(\sigma+s, \widetilde{\varphi}_{\sigma+s}+y_{s}, \widetilde{\varphi}(\sigma+s)+y(s)\right) d s\right|^{p} \leq \\
& \leq\left(\int_{t}^{t+h}\left|F\left(\sigma+s, \widetilde{\varphi}_{\sigma+s}+y_{s}, \widetilde{\varphi}(\sigma+s)+y(s)\right)\right| d s\right)^{p} \leq M^{p}|h|^{p}, \text { o que implica } \\
& \quad\left\|\tau_{|h|} T(y)-T(y)\right\|_{L^{p}((-r+\rho, \alpha-\rho), X)}^{p} \leq \int_{-r+\rho}^{\alpha-\rho} M^{p}|h|^{p} d t \leq M^{p}|h|^{p}(\alpha+r-2 \rho) .
\end{aligned}
$$

$\operatorname{Logo}\left\|\tau_{h} T(y)-T(y)\right\|_{L^{p}((-r+\rho, \alpha-\rho), X)} \leq M|h|(\alpha+r-2 \rho)^{1 / p}$.

Observemos também que,

$$
\begin{gathered}
\|T(y)\|_{L^{p}}^{p}=\int_{-r}^{0}|T(y)(t)|^{p} d t+\int_{0}^{\alpha}|T(y)(t)|^{p} d t=\int_{0}^{\alpha}|T(y)(t)|^{p} d t= \\
=\int_{0}^{\alpha}\left|\int_{0}^{t} F\left(\sigma+s, \widetilde{\varphi}_{\sigma+s}+y_{s}, \widetilde{\varphi}(\sigma+s)+y(s)\right) d s\right|^{p} d t \leq \\
\leq \int_{0}^{\alpha}\left(\int_{0}^{t}\left|F\left(\sigma+s, \widetilde{\varphi}_{\sigma+s}+y_{s}, \widetilde{\varphi}(\sigma+s)+y(s)\right)\right| d s\right)^{p} d t \leq M^{p} \int_{0}^{\alpha} t^{p} d t=\frac{M^{p}}{p+1} \alpha^{p+1} .
\end{gathered}
$$

Logo $\|T(y)\|_{L^{p}} \leq M\left(\alpha^{p+1} /(p+1)\right)^{1 / p}$.

Também, 
- $T(y)$ é absolutamente contínua em $I_{\alpha}$,

- $|T(y)(t)| \leq \int_{0}^{t}\left|F\left(\sigma+s, \widetilde{\varphi}_{\sigma+s}+y_{s}, \widetilde{\varphi}(\sigma+s)+y(s)\right)\right| d s \leq M \alpha$ para todo $t \in I_{\alpha}$ e

$$
\begin{gathered}
\int_{(-r, \alpha) \backslash(-r+\rho, \alpha-\rho)}|T(y)(t)|^{p} d t=\int_{\alpha-\rho}^{\alpha}|T(y)(t)|^{p} d t= \\
=\int_{\alpha-\rho}^{\alpha}\left|\int_{0}^{t} F\left(\sigma+s, \widetilde{\varphi}_{\sigma+s}+y_{s}, \widetilde{\varphi}(\sigma+s)+y(s)\right) d s\right|^{p} d t \leq \int_{\alpha-\rho}^{\alpha} M^{p} t^{p} d t= \\
=M^{p}\left[\left(\alpha^{p+1}-(\alpha-\rho)^{p+1}\right) /(p+1)\right]
\end{gathered}
$$

Logo, $\|T(y)\|_{L^{p}((-r, \alpha) \backslash(-r+\rho, \alpha-\rho))} \leq M\left[\left(\alpha^{p+1}-(\alpha-\rho)^{p+1}\right) /(p+1)\right]^{1 / p}$.

Assim, existe $K \subset L^{p}((-r, \alpha), X)$ compacto, com $T: W \times U \times \mathcal{A}(\alpha, \beta, \gamma) \rightarrow K$.

b) Sabemos que

(i) $T(y) \in L^{p}((-r, \alpha), X)$,

(ii) $T(y)_{0}=0$,

(iii) $\|T(y)\|_{L^{p}} \leq M\left(\alpha^{p+1} /(p+1)\right)^{1 / p}$.

Se $\alpha \leq \min \left\{\left[\beta(p+1)^{1 / p} / M\right]^{p / p+1}, \gamma / M\right\}$, então $|T(y)(t)| \leq \gamma$ e $\left\|T(y)_{t}\right\|_{p} \leq \beta$, para todo $t \in I_{\alpha}$.

Portanto, temos $T: W \times U \times \mathcal{A}(\alpha, \beta, \gamma) \rightarrow \mathcal{A}(\alpha, \beta, \gamma)$.

c) Provemos agora que $T$ é contínua, ou seja, tomemos uma seqüência

$$
\left(\sigma^{k}, \varphi^{k}, \xi^{k}, F^{k}, y^{k}\right) \text { e }\left(\sigma^{0}, \varphi^{0}, \xi^{0}, F^{0}, y^{0}\right) \in W \times U \times \mathcal{A}(\alpha, \beta, \gamma)
$$

tais que $\left(\sigma^{k}, \varphi^{k}, \xi^{k}, F^{k}, y^{k}\right) \rightarrow\left(\sigma^{0}, \varphi^{0}, \xi^{0}, F^{0}, y^{0}\right)$ quando $k \rightarrow \infty$ e mostremos que

$$
T\left(\sigma^{k}, \varphi^{k}, \xi^{k}, F^{k}, y^{k}\right) \rightarrow T\left(\sigma^{0}, \varphi^{0}, \xi^{0}, F^{0}, y^{0}\right) \text { quando } k \rightarrow \infty \text {. }
$$


Sabemos que $T\left(\sigma^{k}, \varphi^{k}, \xi^{k}, F^{k}, y^{k}\right) \in K$ que é compacto. Assim, existem uma subseqüência, que denotaremos da mesma forma, e $g \in K$ tais que

$$
\left\|T\left(\sigma^{k}, \varphi^{k}, \xi^{k}, F^{k}, y^{k}\right)-g\right\|_{L^{p}} \rightarrow 0 \text { quando } k \rightarrow \infty
$$

Por outro lado,

$$
T\left(\sigma^{k}, \varphi^{k}, \xi^{k}, F^{k}, y^{k}\right)(t)=\int_{0}^{t} F^{k}\left(\sigma^{k}+s, \widetilde{\varphi}_{\sigma^{k}+s}^{k}+y_{s}^{k}, \widetilde{\varphi}^{k}\left(\sigma^{k}+s\right)+y^{k}(s)\right) d s
$$

e $\left.F^{k}\left(\sigma^{k}+s, \widetilde{\varphi}_{\sigma^{k}+s}^{k}+y_{s}^{k}, \widetilde{\varphi}^{k}\left(\sigma^{k}+s\right)+y^{k}(s)\right) \rightarrow F^{0}\left(\sigma^{0}+s, \widetilde{\varphi}_{\sigma^{0}+s}^{0}+y_{s}^{0}, \widetilde{\varphi}^{0}\left(\sigma^{0}+s\right)+y^{0}(s)\right)\right)$, para todo $s \in I_{\alpha}$, quando $k \rightarrow \infty$.

O Lema 1.4 nos diz que todas as funções $F^{k}$ acima, a partir de um $k$ suficientemente grande, são uniformemente limitadas. Sendo assim, pelo Teorema da Convergência Dominada,

$$
\begin{aligned}
\int_{0}^{t} F^{k}\left(\sigma^{k}+s, \widetilde{\varphi}_{\sigma^{k}+s}^{k}+\right. & \left.\left.y_{s}^{k}, \widetilde{\varphi}^{k}\left(\sigma^{k}+s\right)+y^{k}(s)\right)\right) d s \rightarrow \\
& \left.\rightarrow \int_{0}^{t} F^{0}\left(\sigma^{0}+s, \widetilde{\varphi}_{\sigma^{0}+s}^{0}+y_{s}^{0}, \widetilde{\varphi}^{0}\left(\sigma^{0}+s\right)+y^{0}(s)\right)\right) d s
\end{aligned}
$$

quando $k \rightarrow \infty$, o que implica $T\left(\sigma^{k}, \varphi^{k}, \xi^{k}, F^{k}, y^{k}\right)(t) \rightarrow T\left(\sigma^{0}, \varphi^{0}, \xi^{0}, F^{0}, y^{0}\right)(t)$ q.t.p. em $(-r, \alpha)$. Assim, $T\left(\sigma^{0}, \varphi^{0}, \xi^{0}, F^{0}, y^{0}\right) \in L^{p}((-r, \alpha), X)$ e

$$
\left\|T\left(\sigma^{k}, \varphi^{k}, \xi^{k}, F^{k}, y^{k}\right)-T\left(\sigma^{0}, \varphi^{0}, \xi^{0}, F^{0}, y^{0}\right)\right\|_{L^{p}} \rightarrow 0 \text { quando } k \rightarrow \infty .
$$

Afirmação: Dada uma seqüência $\left(z_{n}\right)$ em $\hat{K}$, onde $n=1,2, \ldots$ e $\hat{K}$ é compacto, se qualquer subseqüência convergente de $\left(z_{n}\right)$ converge para $z$, então a seqüência $\left(z_{n}\right)$ converge para $z$.

Deste modo, vimos que, toda subseqüência convergente de $T\left(\sigma^{k}, \varphi^{k}, \xi^{k}, F^{k}, y^{k}\right)$ converge para $T\left(\sigma^{0}, \varphi^{0}, \xi^{0}, F^{0}, y^{0}\right)$. Assim, a seqüência $T\left(\sigma^{k}, \varphi^{k}, \xi^{k}, F^{k}, y^{k}\right)$ converge para $T\left(\sigma^{0}, \varphi^{0}, \xi^{0}, F^{0}, y^{0}\right)$. Portanto, $T$ é contínua.

No interesse da independência deste trabalho, recordamos a seguir o Teorema de Schauder. 
Teorema 1.2 Se U é um subconjunto fechado, limitado e convexo de um espaço de Banach $Y$ e $T: U \rightarrow U$ uma aplicação completamente contínua, então $T$ tem um ponto fixo em $U$.

Teorema 1.3 Suponhamos $\Omega$ um subconjunto aberto em $\mathbb{R} \times L^{p} \times X$ e $F^{0} \in C(\Omega, X)$. Se $(\sigma, \varphi, \xi) \in \Omega$, então existe uma solução da equação $\dot{x}(t)=F^{0}\left(t,{ }^{t} x\right)$ passando por $(\sigma, \varphi, \xi)$. Mais geralmente, se $W \subset \Omega$ é compacto e $F^{0} \in C(\Omega, X)$, existem vizinhanças $V \subset \Omega$ de $W, U \subset C^{0}(V, X)$ de $F^{0}$ e um número $\alpha>0$ tais que $F^{0} \in C^{0}(V, X)$ e para todo $(\sigma, \varphi, \xi) \in W$ e $F \in U$, existe uma solução $x(\sigma, \varphi, \xi, F)$ da equação $\dot{x}(t)=F\left(t,{ }^{t} x\right)$ por $(\sigma, \varphi, \xi)$ em $(\sigma-r, \sigma+\alpha)$.

Prova Façamos a prova para o compacto $W=\{(\sigma, \varphi, \xi)\}$. Pelo Lema 1.5

$$
T\left(\sigma, \varphi, \xi, F^{0}, \cdot\right): \mathcal{A}(\alpha, \beta, \gamma) \rightarrow \mathcal{A}(\alpha, \beta, \gamma)
$$

Mostremos que $\mathcal{A}(\alpha, \beta, \gamma)$ é um subconjunto fechado, limitado e convexo do espaço de Banach $L^{p}((-r, \alpha), X)$.

- $\mathcal{A}(\alpha, \beta, \gamma)$ é fechado.

De fato. Seja $y_{n} \in \mathcal{A}(\alpha, \beta, \gamma), n=1,2, \ldots$, tal que $\left\|y_{n}-y\right\|_{L^{p}} \rightarrow 0$ quando $n \rightarrow \infty$. Mostremos que $y \in \mathcal{A}(\alpha, \beta, \gamma)$. Sabemos que $y_{n}(t)=0$ q.t.p. $(-r, 0)$. Como $\left\|y_{n}-y\right\|_{L^{p}} \rightarrow 0$ temos que

$$
\left\|y_{n}-y\right\|_{p}^{p}=\int_{(-r, 0)}\left|y_{n}-y\right|^{p} d t \leq \int_{(-r, \alpha)}\left|y_{n}-y\right|^{p} d t=\left\|y_{n}-y\right\|_{L^{p}}^{p} \rightarrow 0,
$$

quando $n \rightarrow \infty$.

Mas $\left.y_{n}\right|_{(-r, 0)}=0 \in L^{p}$, o que implica $y=0 \in L^{p}$, ou seja, $y_{0}(t)=0$ q.t.p. $(-r, 0)$. Seja $\varepsilon>0$ dado. Existe um número natural $N_{0}$ tal que $\left\|y_{n}-y\right\|_{L^{p}}<\varepsilon$, para $n \geq N_{0}$. Assim,

$$
\left\|y_{t}\right\|_{p} \leq\left\|y_{t}-y_{n_{t}}\right\|_{p}+\left\|y_{n_{t}}\right\|_{p} \leq\left\|y-y_{n}\right\|_{L^{p}}+\left\|y_{n_{t}}\right\|_{p}<\varepsilon+\beta
$$


e existe uma subseqüência $\left(y_{n_{j}}\right)$ tal que $y_{n_{j}}$ converge para $y$ q.t.p. $(-r, \alpha)$, veja por exemplo [2]. Como $y_{n_{j}}$ e $y$ são contínuas em $I_{\alpha}, y_{n_{j}}$ converge pontualmente para $y$ em $I_{\alpha} \mathrm{e}$

$$
|y(t)| \leq\left|y(t)-y_{n_{j}}(t)\right|+\left|y_{n_{j}}(t)\right|<\varepsilon+\gamma, \quad \text { para todo } \quad t \in I_{\alpha} .
$$

Da arbitrariedade de $\varepsilon$ concluímos que $\left\|y_{t}\right\|_{p} \leq \beta$ e $|y(t)| \leq \gamma$, para todo $t \in I_{\alpha}$. Escolha o representante de $y$ tal que $y(0)=0$.

- $\mathcal{A}(\alpha, \beta, \gamma)$ é limitado.

De fato. Como $\alpha<\infty$, existe $k=k(\alpha) \in \mathbb{N}$ e $k<\infty$ tal que $\alpha-(k+1) r \leq r$. Assim, podemos escrever

$$
\int_{(-r, \alpha)}|y(t)|^{p} d t=\int_{(-r, 0)}+\int_{(0, r)}+\cdots+\int_{(k r,(k+1) r)}+\int_{((k+1) r, \alpha)} .
$$

Temos

$$
\int_{0}^{r}|y(t)|^{p} d t=\int_{-r}^{0}|y(r+\theta)|^{p} d \theta=\int_{-r}^{0}\left|y_{r}(\theta)\right|^{p} d \theta=\left\|y_{r}\right\|_{p}^{p} \leq \beta^{p} .
$$

Analogamente,

$$
\int_{r}^{2 r}|y(t)|^{p} d t=\left\|y_{2 r}\right\|_{p}^{p} \leq \beta^{p}, \cdots, \int_{k r}^{(k+1) r}|y(t)|^{p} d t=\left\|y_{(k+1) r}\right\|_{p}^{p} \leq \beta^{p} .
$$

Também,

$$
\int_{(k+1) r}^{\alpha}|y(t)|^{p} d t \leq \int_{\alpha-r}^{\alpha}|y(t)|^{p} d t=\left\|y_{\alpha}\right\|_{p}^{p} \leq \beta^{p} .
$$

Portanto, para todo $y \in \mathcal{A}(\alpha, \beta, \gamma)$,

$$
\|y\|_{L^{p}}^{p} \leq(k+1) \beta^{p}+\beta^{p}=(k+2) \beta^{p}<\infty,
$$

ou seja,

$$
\|y\|_{L^{p}} \leq(k+2)^{1 / p} \beta<\infty .
$$


- $\mathcal{A}(\alpha, \beta, \gamma)$ é convexo.

De fato. Sejam $w_{1}, w_{2} \in \mathcal{A}(\alpha, \beta, \gamma)$ e mostremos que $w=(1-\lambda) w_{1}+\lambda w_{2}$ pertence à $\mathcal{A}(\alpha, \beta, \gamma)$, para todo $\lambda \in[0,1]$. Então 
(i) $w(\theta)=(1-\lambda) w_{1}(\theta)+\lambda w_{2}(\theta)=0$ q.t.p. $(-r, 0)$,

(ii) $\left\|w_{t}\right\|_{p} \leq(1-\lambda)\left\|\left(w_{1}\right)_{t}\right\|_{p}+\lambda\left\|\left(w_{2}\right)_{t}\right\|_{p} \leq \beta$, para todo $t \in I_{\alpha}$,

(iii) $w(0)=(1-\lambda) w_{1}(0)+\lambda w_{2}(0)=0$ e

(iv) $|w(t)| \leq(1-\lambda)\left|w_{1}(t)\right|+\lambda\left|w_{2}(t)\right| \leq \gamma$, para todo $t \in I_{\alpha}$.

Portanto, $\mathcal{A}(\alpha, \beta, \gamma)$ é fechado, limitado e convexo em $\left.L^{p}(-r, \alpha), X\right)$.

Do Lema 1.5, sabemos que $T$ é contínua. Seja $L \subset \mathcal{A}(\alpha, \beta, \gamma)$ limitado. Temos que $T\left(\sigma, \varphi, \xi, F^{0}, L\right) \subset K$, sendo $K$ compacto. Assim, $\overline{T\left(\sigma, \varphi, \xi, F^{0}, L\right)}$ é compacto. Logo $T$ é completamente contínua.

Pelo Teorema 1.2, $T$ possui um ponto fixo em $\mathcal{A}(\alpha, \beta, \gamma)$, ou seja, existe $y$ em $\mathcal{A}(\alpha, \beta, \gamma)$ tal que

$$
\left\{\begin{aligned}
{ }^{0} y & =(0,0) \\
y(t) & =\int_{0}^{t} F^{0}\left(\sigma+s, \widetilde{\varphi}_{\sigma+s}+y_{s}, \widetilde{\varphi}(\sigma+s)+y(s)\right) d s, t \in I_{\alpha} .
\end{aligned}\right.
$$

Portanto, do Lema 1.3, existe uma solução $x$ de (1.2) passando por $(\sigma, \varphi, \xi)$ em $\left.L^{p}(\sigma-r, \sigma+\alpha), X\right)$.

\subsection{Dependência contínua}

Teorema 1.4 Suponha $\Omega$ um subconjunto aberto de $\mathbb{R} \times L^{p} \times X,\left(\sigma^{0}, \varphi^{0}, \xi^{0}\right)$ em $\Omega$, $F^{0} \in C(\Omega, X)$ e $x^{0}$ solução de (1.5) passando por $\left(\sigma^{0}, \varphi^{0}, \xi^{0}\right)$, que existe e é única em $\left(\sigma^{0}-r, \alpha^{0}\right)$. Seja $W^{0} \subset \Omega$ o subconjunto compacto definido por

$$
W^{0}=\left\{\left(t, x_{t}^{0}, x^{0}(t)\right) \mid t \in\left[\sigma^{0}, \alpha^{1}\right]\right\}
$$

onde $\sigma^{0}<\alpha^{1}<\alpha^{0}$, e seja $V^{0}$ vizinhança de $W^{0}$ em $\Omega$ onde $F^{0}$ é limitada. Se $\left(\sigma^{k}, \varphi^{k}, \xi^{k}, F^{k}\right), k=1,2, \ldots$, é uma seqüência que satisfaz

$$
\sigma^{k} \rightarrow \sigma^{0}, \quad \xi^{k} \rightarrow \xi^{0}, \quad\left\|\varphi^{k}-\varphi^{0}\right\|_{p} \rightarrow 0 \quad \text { e } \quad\left|F^{k}-F^{0}\right|_{V^{0}} \rightarrow 0
$$


quando $k \rightarrow \infty$, então existe um número natural $k_{0}$ tal que para $k \geq k_{0}$ cada solução $x^{k}=x^{k}\left(\sigma^{k}, \varphi^{k}, \xi^{k}, F^{k}\right)$ por $\left(\sigma^{k}, \varphi^{k}, \xi^{k}\right)$ de (1.5) existe q.t.p. $\left(\sigma^{k}-r, \alpha^{1}\right)$ e $x^{k}$ converge para $x^{0}$ em $L^{p}\left(\left(\sigma^{0}-r, \alpha^{1}\right), X\right)$. Em outras palavras, para todo $\varepsilon>0$, existe $k^{\prime}=k^{\prime}(\varepsilon)$ tal que $x^{k}$ está definida q.t.p $\left(\sigma^{0}-r+\varepsilon, \alpha^{1}\right)$ para todo $k \geq k^{\prime}$ e $x^{k}$ converge para $x^{0}$ em $L^{p}\left(\left(\sigma^{0}-r+\varepsilon, \alpha^{1}\right), X\right)$ (esta afirmação vem do fato que nem todas as $x^{k}$ estão definidas q.t.p em $\left.\left(\sigma^{0}-r, \alpha^{1}\right)\right)$. Além disso, existe $\varepsilon>0$ tal que $x^{k}$ converge para $x^{0}$ uniformemente em $\left[\sigma^{0}+\varepsilon, \alpha^{1}-\varepsilon\right]$. Se existe $\bar{k}$ tal que para todo $k \geq \bar{k}$ temos $\sigma^{k} \leq \sigma^{0}$ então $x^{k}$ converge uniformemente para $x^{0}$ em $\left[\sigma^{0}, \alpha^{1}-\varepsilon\right]$.

Prova Primeiramente mostremos que

$$
W^{0}=\left\{\left(t, x_{t}^{0}, x^{0}(t)\right) \mid t \in\left[\sigma^{0}, \alpha^{1}\right]\right\}
$$

é compacto. Observemos que $W^{0}$ é um subconjunto de $\mathbb{R} \times L^{p} \times X$.

Sejam $A^{0}=\left\{x_{t}^{0} \mid t \in\left[\sigma^{0}, \alpha^{1}\right]\right\}, B^{0}=\left\{x^{0}(t) \mid t \in\left[\sigma^{0}, \alpha^{1}\right]\right\}$ e observemos que $W^{0}=$ $\left[\sigma^{0}, \alpha^{1}\right] \times A^{0} \times B^{0}$. Mostremos que $A^{0}$ e $B^{0}$ são compactos. Para ver que $A^{0}$ é compacto basta observar que $A^{0}$ é imagem de um compacto pela aplicação contínua $t \rightarrow x_{t}$ em $L^{p}$. Quanto a $B^{0}$, como $x^{0}$ é contínua em $\left[\sigma^{0}, \alpha^{1}\right]$, temos também que $B^{0}$ é compacto.

Como $\left(\sigma^{0}, \varphi^{0}, \xi^{0}\right) \in W^{0}$, segue que $W^{0} \cup\left\{\left(\sigma^{k}, \varphi^{k}, \xi^{k}\right) \mid k=1,2, \ldots\right\}$ é compacto.

Como $\left(\sigma^{k}, \varphi^{k}, \xi^{k}\right)$ converge para $\left(\sigma^{0}, \varphi^{0}, \xi^{0}\right)$ em $\mathbb{R} \times L^{p} \times X$ e $\left(\sigma^{0}, \varphi^{0}, \xi^{0}\right)$ pertence a $W^{0}$ que está contido em $V^{0}$, existe $k_{1} \in \mathbb{N}$ tal que se $k \geq k_{1}$ então $\left(\sigma^{k}, \varphi^{k}, \xi^{k}\right) \in V^{0}$. Logo, $W^{\prime}=W^{0} \cup\left\{\left(\sigma^{k}, \varphi^{k}, \xi^{k}\right) \mid k \geq k_{1}\right\}$ é um compacto contido em $V^{0}$. Como $V^{0}$ é aberto, $W^{\prime}$ é um subconjunto compacto de $V^{0}$ e $F^{0} \in C\left(V^{0}, X\right)$, segue do Lema 1.4 que existem $V \subset V^{0}$, vizinhança de $W^{\prime}$, tal que $F^{0} \in C^{0}(V, X), U \subset$ $C^{0}(V, X)$, vizinhança de $F^{0}$, e constante positiva $M$ tal que $|F(\sigma, \varphi, \xi)|<M$, para todo $(\sigma, \varphi, \xi) \in V$ e para toda $F \in U$. Como $F^{k}$ converge para $F^{0}$ e $F^{0} \in U$, existe $k_{2} \in \mathbb{N}$ tal que se $k \geq k_{2}$, então $F^{k} \in U$.

Apliquemos o Teorema 1.3 ao compacto $W=W^{0} \cup\left\{\left(\sigma^{k}, \varphi^{k}, \xi^{k}\right) \mid k \geq k_{0}\right\}$ onde $k_{0}=$ $\max \left\{k_{1}, k_{2}\right\}$. Como $F^{k} \in U$ e $\left(\sigma^{k}, \varphi^{k}, \xi^{k}\right) \in W$ para $k \geq k_{0}$, o Teorema 1.3 nos garante a existência de um $\alpha>0$ tal que cada uma das soluções $x^{k}\left(\sigma^{k}, \varphi^{k}, \xi^{k}, F^{k}\right)$ existe em $\left(\sigma^{k}-r, \sigma^{k}+\alpha\right)$, onde $\alpha$ não depende de $k$. 
Para $k=0$ e todo $k \geq k_{0}$, seja $y^{k}(t)=x^{k}\left(\sigma^{k}+t\right)-\widetilde{\varphi}^{k}\left(\sigma^{k}+t\right)$ q.t.p. se $t \in(-r, 0)$ e $y^{k}(t)=x^{k}\left(\sigma^{k}+t\right)-\widetilde{\varphi}^{k}\left(\sigma^{k}+t\right)$ se $t \in[0, \alpha)$. Então $y^{k}$ é solução de

$$
\left\{\begin{array}{l}
y_{0}=0 \\
y(t)=\int_{0}^{t} F^{k}\left(\sigma^{k}+s, \widetilde{\varphi}_{\sigma^{k}+s}+y_{s}, \widetilde{\varphi}\left(\sigma^{k}+s\right)+y(s)\right) d s, \quad t \in[0, \alpha) .
\end{array}\right.
$$

Pelo Lema 1.5, $y^{k}=T\left(\sigma^{k}, \varphi^{k}, \xi^{k}, F^{k}, y^{k}\right)$ e pertence a um compacto de $L^{p}((-r, \alpha), X)$. Logo, para $k \geq k_{0}$, existem uma subseqüência $\left(y^{k_{j}}\right)$ de $\left(y^{k}\right)$ e um elemento $y^{*}$ de $L^{p}((-r, \alpha), X)$ tais que $\left\|y^{k_{j}}-y^{*}\right\|_{L^{p}} \rightarrow 0$ quando $k \rightarrow \infty$.

Como $\left(\sigma^{k_{j}}, \varphi^{k_{j}}, \xi^{k_{j}}, F^{k_{j}}, y^{k_{j}}\right) \rightarrow\left(\sigma^{0}, \varphi^{0}, \xi^{0}, F^{0}, y^{*}\right)$ e $T$ é contínua, temos

$$
\left\|T\left(\sigma^{k_{j}}, \varphi^{k_{j}}, \xi^{k_{j}}, F^{k_{j}}, y^{k_{j}}\right)-T\left(\sigma^{0}, \varphi^{0}, \xi^{0}, F^{0}, y^{*}\right)\right\|_{L^{p}} \rightarrow 0, \quad \text { quando } \quad k \rightarrow \infty
$$

e, como $y^{k_{j}}=T\left(\sigma^{k_{j}}, \varphi^{k_{j}}, \xi^{k_{j}}, F^{k_{j}}, y^{k_{j}}\right)$, temos

$$
\left\|y^{k_{j}}-T\left(\sigma^{0}, \varphi^{0}, \xi^{0}, F^{0}, y^{*}\right)\right\|_{L^{p}} \rightarrow 0, \quad \text { quando } \quad k \rightarrow \infty
$$

Logo,

$$
T\left(\sigma^{0}, \varphi^{0}, \xi^{0}, F^{0}, y^{*}\right)=y^{*}
$$

Como $x^{0}$ é a única solução do PVI

$$
\left\{\begin{array}{l}
x_{\sigma^{0}}=\varphi^{0} \\
x(t)=\xi^{0}+\int_{\sigma^{0}}^{t} F^{0}\left(s,{ }^{s} x\right) d s, \quad t \in\left[\sigma^{0}, \sigma^{0}+\alpha\right)
\end{array}\right.
$$

segue que $y^{0}$ é a única solução de $y=T\left(\sigma^{0}, \varphi^{0}, \xi^{0}, F^{0}, y\right)$. Logo, $y^{*}=y^{0}$.

Visto que a seqüência $\left(y^{k}\right)_{k \geq k_{0}}$ possui uma subseqüência convergente para $y^{0} \mathrm{e}$ como qualquer subseqüência convergente de $\left(y^{k}\right)_{k \geq k_{0}}$ deve convergir para $y^{0}$, segue que a seqüência toda converge para $y^{0}$ em $L^{p}((-r, \alpha), X)$.

Como para $k=0$ e $k \geq k_{0}, x^{k}$ existe q.t.p. $\left(\sigma^{k}-r, \sigma^{k}+\alpha\right)$ e $\sigma^{k}$ converge para $\sigma^{0}$, então dado $\varepsilon>0$ existe $k_{1}^{\prime}=k_{1}^{\prime}(\varepsilon)$ tal que para $k=0$ e $k \geq k_{1}^{\prime}$, $x^{k}$ existe q.t.p. $\left(\sigma^{0}-r+\varepsilon, \sigma^{0}+\alpha-\varepsilon\right)$. 
Como $\left\|\varphi^{k}-\varphi^{0}\right\|_{p} \rightarrow 0$ e $\xi^{k} \rightarrow \xi^{0}$, quando $k \rightarrow \infty$, temos

$$
\left\|\widetilde{\varphi}^{k}\left(\sigma^{k}+\cdot\right)-\widetilde{\varphi}^{0}\left(\sigma^{0}+\cdot\right)\right\|_{L^{p}} \rightarrow 0, \quad \text { quando } \quad k \rightarrow \infty .
$$

Então temos

$$
\begin{aligned}
& \left\|x^{k}\left(\sigma^{k}+\cdot\right)-x^{0}\left(\sigma^{0}+\cdot\right)\right\|_{L^{p}}=\left\|y^{k}+\widetilde{\varphi}^{k}\left(\sigma^{k}+\cdot\right)-y^{0}-\widetilde{\varphi}^{0}\left(\sigma^{0}+\cdot\right)\right\|_{L^{p}} \leq \\
& \quad \leq\left\|y^{k}-y^{0}\right\|_{L^{p}}+\left\|\widetilde{\varphi}^{k}\left(\sigma^{k}+\cdot\right)-\widetilde{\varphi}^{0}\left(\sigma^{0}+\cdot\right)\right\|_{L^{p}} \rightarrow 0, \text { quando } k \rightarrow \infty
\end{aligned}
$$

ou seja, $\left\|x^{k}\left(\sigma^{k}+\cdot\right)-x^{0}\left(\sigma^{0}+\cdot\right)\right\|_{L^{p}} \rightarrow 0$, quando $k \rightarrow \infty$. Logo, para $k \geq k_{1}^{\prime}$, temos $\left\|x^{k}-x^{0}\right\|_{L^{p}\left(\left(\sigma^{0}-r+\varepsilon, \sigma^{0}+\alpha-\varepsilon\right), X\right)} \rightarrow 0$, quando $k \rightarrow \infty$.

Se $\sigma^{0}+\alpha>\alpha^{1}$, o teorema está demonstrado. Se $\sigma^{0}+\alpha \leq \alpha^{1}$, existe $k_{2}^{\prime}$ tal que, para todo $k \geq k_{2}^{\prime}$, temos $\sigma^{k}+\alpha \leq \alpha^{1}$.

Para $k \geq k_{2}^{\prime}$ e $k=0$, definimos $\sigma_{1}^{k}=\sigma^{k}+\alpha, \varphi_{1}^{k}=y_{\sigma_{1}^{k}}^{k}$ q.t.p. e $\xi_{1}^{k}=y^{k}\left(\sigma_{1}^{k}\right)$. Então,

$$
\sigma_{1}^{k} \rightarrow \sigma_{1}^{0}, \quad\left\|\varphi_{1}^{k}-\varphi_{1}^{0}\right\|_{p} \rightarrow 0 \quad \text { e } \quad \xi_{1}^{k} \rightarrow \xi_{1}^{0}, \quad \text { quando } \quad k \rightarrow \infty .
$$

Se $\sigma_{0}+\alpha<\alpha_{1}$, podemos repetir os argumentos acima tomando $\sigma_{0}+\alpha$ no papel de $\sigma_{0}$. Como existe um limitante inferior positivo para $\alpha$, após um número finito de repetições chegamos à existência da solução em $\left[\sigma_{0}-r+\varepsilon, \alpha_{1}\right)$.

Dadas as soluções $x^{k}$ em $L^{p}\left(\left[\sigma^{k}, \alpha^{1}\right), X\right)$, sabemos que $x(t)=\xi^{k}+\int_{\sigma^{k}}^{t} F^{k}\left(s,{ }^{s} x\right) d s$ e que as correspondentes soluções $y^{k}$ em $L^{p}\left(\left[0, \alpha^{1}\right), X\right)$ são dadas por

$$
y^{k}(t)=\int_{0}^{t} F^{k}\left(\sigma^{k}+s, \widetilde{\varphi}_{\sigma^{k}+s}^{k}+y_{s}^{k}, \widetilde{\varphi}^{k}\left(\sigma^{k}+s\right)+y^{k}(s)\right) d s
$$

que pertencem a $C([0, \overline{\bar{\alpha}}], X)$, onde $\overline{\bar{\alpha}}<\alpha^{1}$.

Temos também que as $F^{k}, k=0,1,2, \ldots$, são uniformemente limitadas em $V$. Assim,

$$
\left|y^{k}(t)-y^{k}\left(t_{0}\right)\right| \leq \int_{t_{0}}^{t}\left|F^{k}\left(\sigma^{k}+s, \widetilde{\varphi}_{\sigma^{k}+s}^{k}+y_{s}^{k}, \widetilde{\varphi}^{k}\left(\sigma^{k}+s\right)+y^{k}(s)\right)\right| d s \leq M\left|t-t_{0}\right| .
$$

Logo o conjunto formado pelos $y^{k}$ é eqüicontínuo. Esse conjunto também é limitado, pois $\left|y^{k}(t)\right| \leq M \alpha^{1}$. Pelo Teorema de Ascoli-Arzela, existe uma subseqüência $y^{k_{j}}$ 
de $y_{k}$ e $\omega \in C([0, \overline{\bar{\alpha}}], X)$ tais que $y^{k_{j}} \rightarrow \omega$ uniformemente. Como $y^{k_{j}} \rightarrow \omega$ uniformemente, também $y^{k_{j}} \rightarrow \omega$ q.t.p. e, como $\left\|y^{k_{j}}\right\|_{L^{p}([0, \overline{\bar{\alpha}}], X)} \leq M \alpha^{1}$, temos pelo Teorema da Convergência Dominada que $\omega$ é integrável e

$$
\left\|y^{k_{j}}-\omega\right\|_{L^{p}([0, \overline{\bar{\alpha}}], X)} \rightarrow 0, \quad \text { quando } \quad k \rightarrow \infty .
$$

Sabemos também que

$$
\left\|y^{k_{j}}-y^{0}\right\|_{L^{p}([0, \overline{\bar{\alpha}}], X)} \rightarrow 0, \quad \text { quando } \quad k \rightarrow \infty .
$$

Pela unicidade do limite $y^{0}=\omega$ q.t.p., mas como são contínuas em $[0, \overline{\bar{\alpha}}]$ elas são iguais em $[0, \overline{\bar{\alpha}}]$.

Visto que cada subseqüência de $y^{k}$ tem uma subseqüência convergente que deve convergir para $y^{0}$, segue que a seqüência toda converge para $y^{0}$ uniformemente em $[0, \overline{\bar{\alpha}}]$.

Voltando para as correspondentes soluções $x^{k}$, onde

$$
x^{k}\left(\sigma^{k}+t\right)=y^{k}(t)+\widetilde{\varphi}^{k}\left(\sigma^{k}+t\right)=y^{k}(t)+\xi^{k}, \quad t \in[0, \overline{\bar{\alpha}}],
$$

temos que existe $\varepsilon>0$ tal que $x^{k}$ converge para $x^{0}$ uniformemente em $\left[\sigma^{0}+\varepsilon, \alpha^{1}-\varepsilon\right]$.

Observe que, se existe $\bar{k}$ tal que para todo $k \geq \bar{k}, \sigma^{k} \leq \sigma^{0}$, então $x^{k}$ converge para $x^{0}$ uniformemente em $\left[\sigma^{0}, \alpha^{1}-\varepsilon\right]$.

Observação 1.2 O intervalo $\left(\sigma^{0}-r+\varepsilon, \alpha^{1}\right)$ poderia ser trocado por $\left(\sigma^{0}-r+\varepsilon, \alpha^{1}\right]$. Neste caso, $\overline{\bar{\alpha}}=\alpha^{1}$.

\subsection{Unicidade}

Nesta seção trataremos da unicidade de solução da equação $\dot{x}(t)=F\left(t,{ }^{t} x\right)$ passando por $(\sigma, \varphi, \xi)$. Para isso, precisaremos da seguinte definição. 
Definição 1.8 Seja $W$ um subconjunto de $\mathbb{R} \times L^{p} \times X$. Dizemos que a aplicação $F: W \rightarrow X$ é lipschtiziana em $\varphi$ se $F$ satisfaz

$$
\left|F\left(t, \varphi_{1}, \xi\right)-F\left(t, \varphi_{2}, \xi\right)\right| \leq k\left\|\varphi_{1}-\varphi_{2}\right\|_{p}
$$

$\operatorname{para}\left(t, \varphi_{j}, \xi\right) \in W, j=1,2$, e algum $k>0$

Teorema 1.5 Seja $\Omega$ um subconjunto aberto de $\mathbb{R} \times L^{p} \times X, F: \Omega \rightarrow X$ uma aplicação contínua e lipschitziana em $\varphi$ em cada conjunto compacto contido em $\Omega$. Se $(\sigma, \varphi, \xi) \in \Omega$, então existem $\alpha>0$ e uma única solução da equação $\dot{x}(t)=F\left(t,{ }^{t} x\right)$ por $(\sigma, \varphi, \xi)$ em $(\sigma-r, \sigma+\alpha)$.

Prova A existência segue do Teorema 1.3. Suponhamos que $x$ e $y$ sejam soluções da Equação (1.5) em $(\sigma-r, \sigma+\alpha) \operatorname{com} x_{\sigma}=y_{\sigma}=\varphi$ e $x(\sigma)=y(\sigma)=\xi$. Então

$$
\begin{gathered}
x(t)=\xi+\int_{\sigma}^{t} F\left(s,{ }^{s} x\right) d s \quad \text { para } \quad t \in[\sigma, \sigma+\alpha) \quad \text { e } \\
y(t)=\xi+\int_{\sigma}^{t} F\left(s,{ }^{s} y\right) d s \quad \text { para } t \in[\sigma, \sigma+\alpha) .
\end{gathered}
$$

Assim,

(i) $\left\|x_{\sigma}-y_{\sigma}\right\|_{p}=0$,

(ii) $x(\sigma)-y(\sigma)=0 \mathrm{e}$

(iii) $x(t)-y(t)=\int_{\sigma}^{t}\left[F\left(s,{ }^{s} x\right)-F\left(s,{ }^{s} y\right)\right] d s$, se $t \in[\sigma, \sigma+\alpha)$.

Sejam $W_{x}=\left\{\left(t, x_{t}, x(t)\right) \mid t \in\left[\sigma, \sigma+\alpha_{1}\right]\right\}$ e $W_{y}=\left\{\left(t, y_{t}, y(t)\right) \mid t \in\left[\sigma, \sigma+\alpha_{1}\right]\right\}$, onde $0<\alpha_{1}<\alpha$.

Como $x$ e $y$ são soluções, temos que $W_{x} \subset \Omega$ e $W_{y} \subset \Omega$. Vimos também que $W_{x}$ e $W_{y}$ são compactos, o que implica que $W=W_{x} \cup W_{y}$ é compacto.

Seja $k=k(W) \geq 0$ uma constante de Lipschitz de $F$ em $W$, e $\gamma_{1} \in\left[0, \alpha_{1}\right]$ tal que $k^{p}\left(\gamma_{1}^{p+1} /(p+1)\right)<1$. 


$$
\begin{gathered}
\|x-y\|_{L^{p}\left(\left[\sigma, \sigma+\gamma_{1}\right], X\right)}^{p}= \\
=\int_{\sigma}^{\sigma+\gamma_{1}}|x(t)-y(t)|^{p} d t=\int_{\sigma}^{\sigma+\gamma_{1}}\left|\int_{\sigma}^{t} F\left(s, x^{s}\right) d s-\int_{\sigma}^{t} F\left(s, y^{s}\right) d s\right|^{p} d t \leq \\
\leq \int_{\sigma}^{\sigma+\gamma_{1}}\left(\int_{\sigma}^{t}\left|F\left(s, x^{s}\right)-F\left(s, y^{s}\right)\right| d s\right)^{p} d t \leq \\
\leq \int_{\sigma}^{\sigma+\gamma_{1}}\left(\int_{\sigma}^{t} k\left\|x_{s}-y_{s}\right\|_{p} d s\right)^{p} d t=k^{p} \int_{\sigma}^{\sigma+\gamma_{1}}\left(\int_{\sigma}^{t}\left\|x_{s}-y_{s}\right\|_{p} d s\right)^{p} d t= \\
=k^{p} \int_{\sigma}^{\sigma+\gamma_{1}}\left(\int_{\sigma}^{t}\left(\int_{-r}^{0}\left|x_{s}(\theta)-y_{s}(\theta)\right|^{p} d \theta\right)^{p} d s\right)^{p} d t= \\
=k^{p} \int_{\sigma}^{\sigma+\gamma_{1}}\left(\int_{\sigma}^{t}\left(\int_{-r}^{0}|x(s+\theta)-y(s+\theta)|^{p} d \theta\right)^{1 / p} d s\right)^{p} d t
\end{gathered}
$$

Consideremos $u=s+\theta$. Assim,

$$
\begin{gathered}
\|x-y\|_{L^{p}\left(\left[\sigma, \sigma+\gamma_{1}\right], X\right)}^{p} \leq k^{p} \int_{\sigma}^{\sigma+\gamma_{1}}\left(\int_{\sigma}^{t}\left(\int_{s-r}^{s}|x(u)-y(u)|^{p} d u\right)^{1 / p} d s\right)^{p} d t \leq \\
\leq k^{p} \int_{\sigma}^{\sigma+\gamma_{1}}\left(\int_{\sigma}^{t}\left(\int_{\sigma-r}^{\sigma+\gamma_{1}}|x(u)-y(u)|^{p} d u\right)^{1 / p} d s\right)^{p} d t= \\
=k^{p} \int_{\sigma}^{\sigma+\gamma_{1}}\left(\int_{\sigma}^{t}\|x-y\|_{L^{p}\left(\left[\sigma, \sigma+\gamma_{1}\right], X\right)} d s\right)^{p} d t= \\
=k^{p}\|x-y\|_{L^{p}\left(\left[\sigma, \sigma+\gamma_{1}\right], X\right)}^{p} \int_{\sigma}^{\sigma+\gamma_{1}}\left(\int_{\sigma}^{t} d s\right)^{p} d t=k^{p} \frac{\gamma_{1}^{p+1}}{p+1}\|x-y\|_{L^{p}\left(\left[\sigma, \sigma+\gamma_{1}\right], X\right)}^{p} .
\end{gathered}
$$

Logo, como $k^{p}\left(\gamma_{1}^{p+1} /(p+1)\right)<1$ temos $\|x-y\|_{L^{p}\left(\left[\sigma, \sigma+\gamma_{1}\right], X\right)}=0$.

Portanto, $x=y$ q.t.p. em $\left[\sigma, \sigma+\gamma_{1}\right]$. Como $x$ e $y$ são contínuas neste intervalo segue que $x=y$ em $\left[\sigma, \sigma+\gamma_{1}\right]$.

Se $\gamma_{1} \geq \alpha_{1}$ o teorema está demonstrado, dada a arbitrariedade de $\alpha_{1} \in(0, \alpha)$.

Se não, tomemos $\sigma^{\prime}=\sigma+\gamma_{1}, \varphi^{\prime}=x_{\sigma^{\prime}}=y_{\sigma^{\prime}}$ q.t.p., $\xi^{\prime}=x\left(\sigma^{\prime}\right)=y\left(\sigma^{\prime}\right)$.

Temos que $x$ e $y$ são soluções de $\dot{x}(t)=F\left(t,{ }^{t} x\right)$ passando por $\left(\sigma^{\prime}, \varphi^{\prime}, \xi^{\prime}\right)$ em $\left(\sigma^{\prime}-r, \sigma+\alpha_{1}\right]$. 
Logo

$$
\begin{aligned}
& x(t)=\xi^{\prime}+\int_{\sigma^{\prime}}^{t} F\left(s,{ }^{s} x\right) d s \quad \text { para } \quad t \in\left[\sigma^{\prime}, \sigma+\alpha_{1}\right] \quad \text { e } \\
& y(t)=\xi^{\prime}+\int_{\sigma^{\prime}}^{t} F\left(s,{ }^{s} y\right) d s \quad \text { para } t \in\left[\sigma^{\prime}, \sigma+\alpha_{1}\right] .
\end{aligned}
$$

Escolha $\gamma_{2}>0$ tal que $\gamma_{2} \in\left[0, \alpha_{1}\right]$ e $k^{p}\left(\gamma_{2}^{p+1} /(p+1)\right)<1$. Analogamente ao que foi feito mostramos que $x(t)=y(t) \operatorname{em}\left[\sigma^{\prime}, \sigma^{\prime}+\gamma_{2}\right]=\left[\sigma^{\prime}, \sigma+\gamma_{1}+\gamma_{2}\right]$. Novamente, se $\gamma_{1}+\gamma_{2} \geq \alpha_{1}$, acabou a prova. Se não, continuando este processo, obtemos uma seqüência $\psi_{n}$, de modo que $x(t)=y(t)$ em $\left[\sigma, \sigma+\psi_{n}\right]$, onde $\psi_{n}=\sum_{i=1}^{n} \gamma_{i}$ para qualquer $n \in \mathbb{N}$.

Seja $\gamma^{*}=\sup _{n} \psi_{n}$ e mostremos que $\gamma^{*} \geq \alpha_{1}$.

De fato. Suponhamos $\gamma^{*}<\alpha_{1}$. Temos que $x(t)=y(t)$ em $\left[\sigma, \sigma+\gamma^{*}\right]$.

Tomemos $\sigma^{*}=\sigma+\gamma^{*}, \varphi^{*}=x_{\sigma^{*}}=y_{\sigma^{*}}$ q.t.p. e $\xi^{*}=x\left(\sigma^{*}\right)=y\left(\sigma^{*}\right)$.

Logo $x$ e $y$ são soluções de $\dot{x}(t)=F\left(t,{ }^{t} x\right)$ por $\left(\sigma^{*}, \varphi^{*}, \xi^{*}\right)$, em $\left(\sigma^{*}-r, \sigma+\alpha_{1}\right]$.

Então

$$
\begin{aligned}
& x(t)=\xi^{*}+\int_{\sigma^{*}}^{t} F\left(s,{ }^{s} x\right) d s \quad \text { para } \quad t \in\left[\sigma^{*}, \sigma+\alpha_{1}\right] \quad \text { e } \\
& y(t)=\xi^{*}+\int_{\sigma^{*}}^{t} F\left(s,{ }^{s} y\right) d s \quad \text { para } \quad t \in\left[\sigma^{*}, \sigma+\alpha_{1}\right] .
\end{aligned}
$$

Escolhamos $\bar{\gamma}$ tal que $\bar{\gamma} \in\left[0, \alpha_{1}\right]$ e $k^{p}\left(\bar{\gamma}^{p+1} /(p+1)\right)<1$.

Com precisamente os mesmos argumentos, provamos que $x=y \mathrm{em}\left[\sigma^{*}, \sigma+\gamma^{*}+\bar{\gamma}\right]$.

Como $\sigma^{*}=\sigma+\gamma^{*}$ temos uma contradição. Portanto, $x=y$ em $\left[\sigma, \sigma+\alpha_{1}\right]$. Da arbitrariedade de $\alpha_{1} \in(0, \alpha)$ concluímos que $x=y$ em $[\sigma, \sigma+\alpha)$.

\subsection{Continuação de soluções}

Definição 1.9 Se x é uma solução da equação (1.1) no intervalo $(\sigma-r, \sigma+\alpha), \alpha>0$, dizemos que $\hat{x}$ é uma continuação de $x$, se existe $\hat{\alpha}>\alpha$ tal que

(i) $\hat{x}$ é solução da equação (1.1) em $(\sigma-r, \sigma+\hat{\alpha})$, 
(ii) $\hat{x}=x$ q.t.p. $(\sigma-r, \sigma) e$

(iii) $\hat{x}=x$ em $[\sigma, \sigma+\alpha)$.

Definição 1.10 Dizemos que uma solução $x$ é não continuável se não existe tal continuação, isto é, o intervalo $(\sigma-r, \sigma+\alpha)$ é um intervalo maximal de existência da solução $x$.

Observação 1.3 Se F é contínua, a existência de pelo menos uma solução não continuável da equação (1.2) é garantida pelo Lema de Zorn.

Observação 1.4 O intervalo de existência de qualquer solução não continuável da equação (1.2), sendo F contínua, é um intervalo aberto.

Teorema 1.6 Suponha que $\Omega$ seja um aberto em $\mathbb{R} \times L^{p} \times X$ e $F \in C(\Omega, X)$. Se $x$ é uma solução não continuável da equação (1.2) em $(\sigma-r, b)$, então para qualquer conjunto compacto $W$ em $\Omega$, existe um $t_{W} \in(\sigma, b)$ tal que $\left(t, x_{t}, x(t)\right) \notin W$ para $t_{W} \leq t<b$.

Prova Suponha $b=\infty$. Se $W$ é compacto, existe $M>0$ tal que $(t, \varphi, \xi) \in W$ implica $|t| \leq M$. Basta escolher $t_{W}>M$.

Seja $b<\infty$. Suponhamos que a afirmação do teorema seja falsa. Então existem um compacto $W$ e uma seqüência $\left(t_{k}\right)$ de números reais em $[\sigma, b)$ tal que $t_{k} \rightarrow b^{-}$, quando $k \rightarrow \infty$, e $\left(t_{k}, x_{t_{k}}, x\left(t_{k}\right)\right) \in W, k=1,2,3, \ldots$

Como $W$ é compacto, podemos supor, passando a uma subseqüência se necessário, que $\left(t_{k}, x_{t_{k}}, x\left(t_{k}\right)\right)$ converge para $(b, \bar{\psi}, \bar{\zeta}) \in W$, quando $k \rightarrow \infty$.

Afirmação: $x(t)$ é uniformemente contínua em $[\sigma, b)$. De fato, pois pelo Lema 1.4, existe uma vizinhança $V(W)$ de $W$ tal que $F \in C^{0}(V(W), X)$. Como $(b, \bar{\psi}, \bar{\zeta}) \in W$, existe $V=V(b, \bar{\psi}, \bar{\zeta})$ e $M>0$ tal que $|F(t, \psi, \zeta)|<M$, para todo $(t, \psi, \zeta) \in V$. Como $\left(t_{k}, x_{t_{k}}, x\left(t_{k}\right)\right) \rightarrow(b, \bar{\psi}, \bar{\zeta})$, existe $k_{0}$ tal que para $k>k_{0},\left|F\left(t_{k}, x_{t_{k}}, x\left(t_{k}\right)\right)\right|<M$. Aumentando $M$, se necessário, $\mid F\left(t, x_{t}, x(t) \mid<M\right.$, para todo $t \in[\sigma, b)$. Seja $\varepsilon>0$ dado. Se $\delta_{1}=\varepsilon / 2 M, t, t_{0} \in[\sigma, b)$ e $\left|t-t_{0}\right|<\delta_{1}$, então 


$$
\begin{gathered}
\left|x(t)-x\left(t_{0}\right)\right|=\left|\xi+\int_{\sigma}^{t} F\left(s,{ }^{s} x\right) d s-\xi-\int_{\sigma}^{t_{0}} F\left(s,{ }^{s} x\right) d s\right|=\left|\int_{t_{0}}^{t} F\left(s,{ }^{s} x\right) d s\right| \leq \\
\leq \int_{t_{0}}^{t}\left|F\left(s,{ }^{s} x\right)\right| d s \leq M\left|t-t_{0}\right|<\frac{\varepsilon}{2},
\end{gathered}
$$

ou seja, $x(t)$ é uniformemente contínua em $[\sigma, b)$. Assim $x$ se estende continuamente a $[\sigma, b]$.

Como a função $t \rightarrow x_{t}$ é contínua em $L^{p}$, existe $\delta_{2}>0$ tal que se $\left|t-t_{0}\right|<\delta_{2}$, então $\left\|x_{t}-x_{t_{0}}\right\|_{p}<\varepsilon / 2$. Seja $\delta=\min \left\{\delta_{1}, \delta_{2}\right\}$.

Como $\lim _{k \rightarrow \infty} t_{k}=b^{-}$, existe $k_{0}$ tal que para $k \geq k_{0}$ temos $0<b-t_{k}<\delta / 2$.

Como $\lim _{k \rightarrow \infty} x\left(t_{k}\right)=\zeta$, existe $k_{1}$ tal que para $k \geq k_{1},\left|x\left(t_{k}\right)-\zeta\right|<\varepsilon / 2$.

Como $\left\|x_{t_{k}}-\psi\right\|_{p} \rightarrow 0$, existe $k_{2}$ tal que para $k \geq k_{2},\left\|x_{t_{k}}-\psi\right\|_{p}<\varepsilon / 2$.

Seja $\bar{k}=\max \left\{k_{0}, k_{1}, k_{2}\right\}$. Assim,

$$
b-t_{\bar{k}}<\frac{\delta}{2}, \quad\left|x\left(t_{\bar{k}}\right)-\zeta\right|<\frac{\varepsilon}{2} \quad \text { e } \quad\left\|x_{t_{\bar{k}}}-\psi\right\|_{p}<\frac{\varepsilon}{2} .
$$

Logo, se $t \in[\sigma, b)$ e $0<b-t<\delta / 2$, temos

$$
\left|t-t_{\bar{k}}\right| \leq|t-b|+\left|b-t_{\bar{k}}\right|=(b-t)+\left(b-t_{\bar{k}}\right)<\delta,
$$

o que implica

$$
\left|x(t)-x\left(t_{\bar{k}}\right)\right|<\frac{\varepsilon}{2} \quad \text { e } \quad\left\|x_{t}-x_{t_{\bar{k}}}\right\|_{p}<\frac{\varepsilon}{2} .
$$

Portanto,

$$
\begin{gathered}
|x(t)-\zeta| \leq\left|x(t)-x\left(t_{\bar{k}}\right)\right|+\left|x\left(t_{\bar{k}}\right)-\zeta\right|<\varepsilon \text { o que implica } x(t) \rightarrow \zeta \\
\text { e } \quad\left\|x_{t}-\psi\right\|_{p} \leq\left\|x_{t}-x_{t_{\bar{k}}}\right\|_{p}+\left\|x_{t_{\bar{k}}}-\psi\right\|_{p}<\varepsilon \text { o que implica }\left\|x_{t}-\psi\right\|_{p} \rightarrow 0 .
\end{gathered}
$$

Sabemos que $x(t) \rightarrow x(b)$ e $\left\|x_{t}-x_{b}\right\|_{p} \rightarrow 0$.

Assim, $x(b)=\zeta$ e $x_{b}=\psi$.

Portanto $\left(b, x_{b}, x(b)\right) \in W$ e pelo teorema da existência, temos que existe uma continuação de $x$ a $(\sigma-r, b+a)$, para algum $a>0$, o que é uma contradição. 
Corolário 1.1 Suponha que $\Omega$ seja um aberto em $\mathbb{R} \times L^{p} \times X$ e $F \in C(\Omega, X)$. Se $x$ é uma solução não continuável da equação (1.2) em $(\sigma-r, b)$ e

$$
W=\overline{\left\{\left(t, x_{t}, x(t)\right) \in \mathbb{R} \times L^{p} \times X \mid t \in[\sigma, b)\right\}},
$$

então $W$ compacto implica que existe uma seqüência $\left(t_{k}\right), k=1,2, \ldots$, de números reais, $t_{k} \rightarrow b^{-}$quando $k \rightarrow \infty$ tal que $\left(t_{k}, x_{t_{k}}, x\left(t_{k}\right)\right)$ tende a $\partial \Omega$ quando $k \rightarrow \infty$. Além disso, existe um $(b, \psi, \zeta) \in \mathbb{R} \times L^{p} \times X$ tal que $(b, \psi, \zeta) \in \partial \Omega e\left(t, x_{t}, x(t)\right) \rightarrow(b, \psi, \zeta)$ quando $t \rightarrow b^{-}$.

Prova Seja $W_{1} \stackrel{\text { def }}{=}\left\{\left(t, x_{t}, x(t)\right) \in \mathbb{R} \times L^{p} \times X \mid t \in[\sigma, b)\right\} \cup\left\{\left(b, x_{b}, x(b)\right)\right\}$. Afirmação 1: $W=W_{1}$.

Mostremos primeiro que $W_{1} \subset W$. É claro que se $t \in[\sigma, b)$ então $\left(t, x_{t}, x(t)\right) \in W$. Tomemos $\left(t_{k}\right), k=1,2, \ldots$, uma seqüência tal que $\lim _{k \rightarrow \infty} t_{k}=b^{-}, t_{k} \in[\sigma, b)$.

Como $\left(t_{k}, x_{t_{k}}, x\left(t_{k}\right)\right) \in W, k=1,2, \ldots$, passando a uma subseqüência se necessário, podemos dizer que existe $(b, \psi, \zeta) \in W$ tal que $\left(t_{k}, x_{t_{k}}, x\left(t_{k}\right)\right) \rightarrow(b, \psi, \zeta)$, ou seja,

$$
\left\|x_{t_{k}}-\psi\right\|_{p} \rightarrow 0 \quad \text { e } \quad\left|x\left(t_{k}\right)-\zeta\right| \rightarrow 0, \quad \text { quando } \quad k \rightarrow \infty
$$

Como $W$ é compacto e $F$ é contínua em $W$, temos que $F$ é limitada no conjunto $\left\{\left(t, x_{t}, x(t)\right) \in \mathbb{R} \times L^{p} \times X \mid t \in[\sigma, b)\right\}$. Logo existe $M \geq 0$ tal que $\left|F\left(t,{ }^{t} x\right)\right| \leq M$, para todo $t \in[\sigma, b)$.

Assim $x$ é uniformemente contínua em $[\sigma, b)$, pois

$$
|x(t)-x(s)|=\left|\int_{s}^{t} F\left(u,{ }^{u} x\right) d u\right| \leq \int_{s}^{t}\left|F\left(u,{ }^{u} x\right)\right| d u \leq M(t-s) .
$$

Sendo $\lim _{k \rightarrow \infty} t_{k}=b$, pela continuidade da função $t \rightarrow x_{t}$, temos

$$
\left\|x_{t_{k}}-x_{b}\right\|_{p} \rightarrow 0 \quad \text { quando } \quad k \rightarrow \infty
$$

e, como podemos estender continuamente $x$ ao intervalo $[\sigma, b]$, temos

$$
\left|x\left(t_{k}\right)-x(b)\right| \rightarrow 0 \quad \text { quando } \quad k \rightarrow \infty .
$$


Assim, $\psi=x_{b}$ e $\zeta=x(b)$, o que implica $\left(b, x_{b}, x(b)\right) \in W$. Logo, $W_{1} \subset W$.

Facilmente mostramos que $W \subset W_{1}$.

Afirmação 2: $W \not \subset \Omega$.

De fato, pois se $W \subset \Omega$, pelo Teorema 1.6 existe $t_{W} \in(\sigma, b)$ tal que se $t \in\left[t_{W}, b\right)$ então $\left(t, x_{t}, x(t)\right) \notin W$, o que contraria a definição de $W$.

Como $\left(t, x_{t}, x(t)\right) \in \Omega$, para todo $t \in[\sigma, b)$, pelas afirmações 1 e 2 concluímos que $\left(b, x_{b}, x(b)\right) \notin \Omega$, o que implica que, $\left(b, x_{b}, x(b)\right) \in \partial \Omega$.

Assim, $W \cap \partial \Omega$ é não vazio. Como $\left(b, x_{b}, x(b)\right) \in W$, existe uma seqüência $\left(t_{k}, x_{t_{k}}, x\left(t_{k}\right)\right), k=1,2, \ldots, t_{k} \in[\sigma, b)$ tal que $\left(t_{k}, x_{t_{k}}, x\left(t_{k}\right)\right) \rightarrow\left(b, x_{b}, x(b)\right) \in \partial \Omega$.

Vamos provar agora a segunda afirmação do corolário. Sabemos que existe uma seqüência $\left(t_{k}, x_{t_{k}}, x\left(t_{k}\right)\right)$ tal que $\left(t_{k}, x_{t_{k}}, x\left(t_{k}\right)\right) \rightarrow\left(b, x_{b}, x(b)\right)$ quando $k \rightarrow \infty$, e que $\left(t, x_{t}, x(t)\right) \in \Omega$ para todo $t \in[\sigma, b)$.

Seja $\varepsilon>0$ dado.

Pela continuidade uniforme de $x$ em $[\sigma, b]$, existe $\delta_{1}>0$ tal que se $0<b-t_{k}<\delta_{1} / 2$ e $\left|t-t_{k}\right|<\delta_{1} / 2$ então

$$
\left|x(t)-x\left(t_{k}\right)\right|<\frac{\varepsilon}{2} \quad \text { e } \quad\left|x\left(t_{k}\right)-x(b)\right|<\frac{\varepsilon}{2}
$$

e dado $\varepsilon^{\prime}=\varepsilon / 2 r^{1 / p}$, existe $\delta_{2}>0$ tal que se $\left|t-t_{k}\right|<\delta_{2}$ então

$$
\left|x(t+\theta)-x\left(t_{k}+\theta\right)\right|<\varepsilon^{\prime} \text { q.t.p para } \theta \in[-r, 0]
$$

o que implica $\left\|x_{t}-x_{t_{k}}\right\|_{p}<\varepsilon / 2$.

Seja $\delta / 2=\min \left\{\delta_{1} / 2, \delta_{2}\right\}$ e $k_{0}$ suficientemente grande tal que para $k \geq k_{0}$ temos

$$
0<b-t_{k}<\frac{\delta}{2}, \quad\left|t-t_{k}\right|<\frac{\delta}{2}, \quad \text { e } \quad\left\|x_{t_{k}}-x_{b}\right\|_{p}<\frac{\varepsilon}{2} .
$$

Então,

$$
\begin{gathered}
|x(t)-x(b)| \leq\left|x(t)-x\left(t_{k}\right)\right|+\left|x\left(t_{k}\right)-x(b)\right|<\varepsilon \quad \mathrm{e} \\
\left\|x_{t}-x_{b}\right\|_{p} \leq\left\|x_{t}-x_{t_{k}}\right\|_{p}+\left\|x_{t_{k}}-x_{b}\right\|_{p}<\varepsilon .
\end{gathered}
$$

Portanto, existe $(b, \psi, \zeta)=\left(b, x_{b}, x(b)\right) \in \partial \Omega$ tal que $\left(t, x_{t}, x(t)\right) \rightarrow(b, \psi, \zeta)$ quando $t \rightarrow b^{-}$. 
Como na teoria clássica das E.D.F.R., para garantir que as soluções de (1.2) escapem de subconjuntos limitados e fechados de $\Omega$, é necessário impor condições mais fortes sobre o campo vetorial $F$.

O Teorema 1.7, cuja prova é uma adaptação extraída da teoria clássica, estabelece essas condições.

Teorema 1.7 Suponha que $\Omega$ seja um aberto de $\mathbb{R} \times L^{p} \times X, F: \Omega \rightarrow \mathbb{R}^{n}$ completamente contínua e $x$ solução não continuável da equação $(1.2)$ em $(\sigma-r, b)$. Então, para todo conjunto fechado e limitado $U \subset \Omega$, existe $t_{U} \in(\sigma, b)$ tal que $\left(t, x_{t}, x(t)\right) \notin U$ para $t_{U} \leq t<b$.

Prova Se $b=\infty$ a prova é análoga à do Teorema 1.6.

Suponhamos então $b<\infty$. Vamos supor que a conclusão do teorema não valha, ou seja, existe uma seqüência $\left(t_{k}\right)$ de números reais no intervalo $[\sigma, b), t_{k} \rightarrow b^{-}$, e um conjunto fechado e limitado $U$ tal que $\left(t_{k}, x_{t_{k}}, x\left(t_{k}\right)\right) \in U, k=1,2, \ldots$ Assim, existe $N_{1}>0$ tal que $\left\|x_{t_{k}}\right\|_{p} \leq N_{1}$ e $\left|x\left(t_{k}\right)\right| \leq N_{1}$, para $k=1,2, \ldots$.

Se $x \in L^{p}((\sigma-r, b-\varepsilon), X)$ e $\int_{\sigma-r}^{b}|x(s)|^{p} d s=+\infty$ então existe $\delta>0$ tal que $\int_{b-\delta}^{b}|x(s)|^{p} d s=\infty$, pois $\int_{\sigma-r}^{\sigma}|x(s)|^{p} d s<\infty$ (condição inicial) e $x$ contínua em $[\sigma, b)$ implica $x$ contínua em $[\sigma, b-\delta]$ para qualquer $\delta$, o que implica $\int_{\sigma}^{b-\delta}|x(s)|^{p} d s<\infty$. Logo existe $k$ tal que $\left(t_{k}, x_{t_{k}}, x\left(t_{k}\right)\right) \notin U$, o que é uma contradição.

Consideremos agora $\int_{\sigma-r}^{b}|x(s)|^{p} d s<+\infty$. Sejam

$$
A=\left\{x_{t} \in L^{p}((-r, 0), X) \mid t \in[\sigma, b)\right\} \quad \text { e } \quad B=\{x(t) \in X \mid t \in[\sigma, b)\} .
$$

Temos que $A$ é limitado em $L^{p}((-r, 0), X)$. Afirmação: $x(t)$ é limitada em $[\sigma, b)$.

De fato. Suponhamos, por absurdo, que $x(t)$ não seja limitada em $[\sigma, b)$. Então para qualquer constante $Z>0$, existe $t \in[\sigma, b)$ tal que $|x(t)|>Z$. Numa prova 
análoga à feita no Teorema 1.4, podemos mostrar que para qualquer $t \in[\sigma, b)$, o conjunto

$$
W_{t}=\left\{\left(s, x_{s}, x(s)\right) \mid s \in[\sigma, t]\right\}
$$

é compacto e, portanto, fechado e limitado. Por hipótese, $F$ é completamente contínua e, então $F\left(W_{t}\right)$ é limitado em $X$. Assim, existe uma constante $N>0$ tal que

$$
\left|F\left(s, x_{s}, x(s)\right)\right| \leq N, \quad \text { para todo } s \in[\sigma, t]
$$

Sejam $N_{2}>0, Z=N_{2}(b-\sigma)+N_{1}$, e $t, t_{k} \in[\sigma, b)$ tais que

$$
t_{k}<t, \quad\left|F\left(s, x_{s}, x(s)\right)\right| \leq N_{2}, \quad \text { para todo } \quad s \in[\sigma, t] \quad \text { e } \quad|x(t)|>Z .
$$

Assim,

$$
|x(t)| \leq\left|x(t)-x\left(t_{k}\right)\right|+\left|x\left(t_{k}\right)\right| \leq \int_{t_{k}}^{t}\left|F\left(s,{ }^{s} x\right)\right| d s+N_{1} \leq N_{2}(b-\sigma)+N_{1},
$$

ou seja, $|x(t)| \leq Z$, o que é uma contradição. Portanto $B$ é limitado.

Definamos $W=\left\{\left(t, x_{t}, x(t)\right) \mid t \in[\sigma, b)\right\}$. Então $W$ é limitado, o que implica $\bar{W}$ fechado e limitado. Como $F$ é completamente contínua, temos $F(\bar{W})$ limitado em $X$. Assim, existe uma constante $M>0$ tal que

$$
|F(t, \varphi, \xi)| \leq M, \quad \text { para todo } \quad(t, \varphi, \xi) \in \bar{W}
$$

Sendo assim, $x$ é uniformemente contínua em $[\sigma, b)$, pois

$$
\left|x(t)-x(s) \leq \int_{s}^{t}\right| F\left(u, x_{u}, x(u)\right)|d u \leq M| t-s \mid
$$

Logo, $x$ se estende continuamente à $[\sigma, b]$ e, então $x$ é limitada em $[\sigma, b]$. Assim, $\bar{B}$ é limitado em $X$.

Afirmação: A é relativamente compacto. Para isso usaremos o Teorema de Fréchet-Kolmogorov, veja por exemplo [3].

Dado $\varepsilon>0$ e $w \subset \subset(-r, 0)$, existe $\rho>0$ tal que $w \subset(-r+\rho,-\rho) \subset \subset(-r, 0)$. Seja $\delta=\min \left\{\rho, \varepsilon /\left(M(r-2 \rho)^{1 / p}\right)\right\}$. Então 


$$
\begin{aligned}
& \left\|\tau_{h} x_{t}-x_{t}\right\|_{L^{p}((-r+\rho,-\rho), X)}^{p}=\int_{-r+\rho}^{-\rho}\left|x_{t}(\theta+h)-x_{t}(\theta)\right|^{p} d \theta= \\
& =\int_{-r+\rho}^{-\rho}|x(t+\theta+h)-x(t+\theta)|^{p} d \theta=\int_{t-r+\rho}^{t-\rho}|x(u+h)-x(u)|^{p} d u= \\
& =\int_{t-r+\rho}^{t-\rho}\left|\int_{\sigma}^{u+h} F\left(s,{ }^{s} x\right) d s-\int_{\sigma}^{u} F\left(s,{ }^{s} x\right) d s\right| d u \leq \int_{t-r+\rho}^{t-\rho} \int_{u}^{u+h}\left|F\left(s,{ }^{s} x\right)\right| d s d u \leq \\
& \leq \int_{t-r+\rho}^{t-\rho} M^{p}|h|^{p} d u \leq M^{p}|h|^{p}(r-2 \rho)<\varepsilon^{p}, \quad(1.16)
\end{aligned}
$$

para todo $h \in X$ com $|h|<\delta$ e todo $x_{t} \in A$.

Mostremos agora que existe $w \subset \subset(-r, 0)$ tal que $\left\|x_{t}\right\|_{L^{p}((-r, 0) \backslash w)}<\varepsilon$, para todo $x_{t} \in A$.

Como $x \in L^{p}((\sigma-r, b), X)$ temos que existe $\bar{\delta}>0$ tal que para qualquer conjunto $B$ no qual a medida de $B$ é menor que $\bar{\delta}$, então $\int_{B}|x(u)|^{p} d u<\varepsilon^{p} / 2$.

Seja $w=(-r+\bar{\rho},-\bar{\rho})$ onde $\bar{\rho}<\min \{\bar{\delta}, r\}$. Assim,

$$
\begin{aligned}
\left\|x_{t}\right\|_{L^{p}((-r, 0) \backslash w)}^{p}= & \int_{(-r, 0) \backslash w}\left|x_{t}(\theta)\right|^{p} d \theta= \\
= & \int_{-r}^{-r+\bar{\rho}}|x(t+\theta)|^{p} d \theta+\int_{-\bar{\rho}}^{0}|x(t+\theta)|^{p} d \theta= \\
& =\int_{t-r}^{t-r+\bar{\rho}}|x(u)|^{p} d u+\int_{t-\bar{\rho}}^{t}|x(u)|^{p} d u<\frac{\varepsilon^{p}}{2}+\frac{\varepsilon^{p}}{2}=\varepsilon^{p},
\end{aligned}
$$

para todo $t \in[\sigma, b)$, ou seja, para todo $x_{t} \in A$.

Portanto, $\bar{A}$ e $\bar{B}$ são compactos.

Como $\bar{W}$ está contido no compacto $[\sigma, b] \times \bar{A} \times \bar{B}$ temos que $W$ está contido num compacto, o que contraria o Teorema 1.6. 


\subsection{Comentários finais}

As equações diferenciais a diferenças não estão incluídas entre as estudadas neste capítulo. De fato, se

$$
\dot{x}(t)=f(x(t-1))=f\left(x_{t}(-1)\right),
$$

a menos que tenhamos uma interpretação mais elaborada, não faz sentido escrever $F(t, \varphi, \xi)=G(\varphi)=f(\varphi(-1)), \operatorname{com} \varphi \in L^{p}$.

Se $g$ é lipschitziana, a equação

$$
\dot{x}(t)=\int_{-r}^{-s} g(x(t+\theta)) d \theta=\int_{-r}^{-s} g\left(x_{t}(\theta)\right) d \theta, \quad 0 \leq s<r
$$

pode ser escrita como

$$
\dot{x}(t)=F\left(t,{ }^{t} x\right)
$$

onde $F(t, \varphi, \xi)=G(\varphi)=\int_{-r}^{-s} g(\varphi(\theta)) d \theta$. Aplicando a desigualdade de Hölder concluímos que $F$ é lipschitziana em $\varphi$.

Se $g$ é contínua e limitada, segue do Teorema da Convergência Limitada que $F$ é contínua. 


\section{Capítulo 2}

\section{Equações características e bifurcações de Hopf}

\subsection{Introdução}

Em [9] Nussbaum estuda a equação diferencial escalar com retardamento

$$
\dot{x}(t)=-\frac{\alpha}{\delta} \int_{-1}^{-1+\delta} f(x(t+\theta)) d \theta
$$

onde $\alpha$ é um número real estritamente positivo, $0<\delta \leq 1 / 2$ (em alguns casos esta condição é relaxada para $0<\delta \leq 1)$ e $f: \mathbb{R} \rightarrow \mathbb{R}$ satisfaz a hipótese

(H.1) $f$ contínua; $f^{\prime}(0)=1 ; x f(x)>0$ se $x \neq 0$ e $f(x) \geq-B>-\infty$ se $x \in \mathbb{R}$.

A equação (2.1) foi estudada por Walther em [15] para o caso $\delta=1 / 2$.

Com algum cuidado a equação (2.1) pode ser interpretada para $\delta=0$ como a equação

$$
\dot{x}(t)=-\alpha f(x(t-1))
$$

Com o objetivo de descrever um arcabouço para parte do nosso trabalho, apresentamos a seguir alguns resultados de [9]. 
Na seção 2.3 estudamos uma versão planar da equação (2.1). Provamos a existência de uma seqüência de valores críticos do parâmetro $\alpha$ onde ocorrem bifurcações de Hopf.

\subsection{Uma motivação escalar}

Sabemos que $x=0$ é uma solução da equação (2.1). Logo, podemos esperar que exista uma solução periódica não nula se a solução trivial for instável. Se linearizarmos (2.1) em torno de 0 , teremos

$$
\dot{x}(t)=-\frac{\alpha}{\delta} \int_{-1}^{-1+\delta} x(t+\theta) d \theta
$$

cuja equação característica é dada por

$$
\lambda=-\frac{\alpha}{\delta} \int_{-1}^{-1+\delta} e^{\lambda \theta} d \theta
$$

Soluções da equação (2.4) com parte real positiva indicam instabilidade da origem. O próximo lema estabelece que tais soluções ocorrem para valores do parâmetro $\alpha$ maiores que um certo $\alpha_{0}$.

Lema 2.1 Suponha $0<\delta \leq 1$ e $g:[-1,-1+\delta] \rightarrow \mathbb{R}$ uma função contínua tal que $I=\int_{-1}^{-1+\delta} g(\theta) d \theta>0$. Sejam $b_{j}, b_{j}<b_{j+1}$, as raízes positivas da equação

$$
\int_{-1}^{-1+\delta} g(\theta) \cos (b \theta) d \theta=0
$$

Existe somente uma quantidade enumerável de raízes dessa equação e estas não têm ponto de acumulação. Defina

$$
\beta_{j}=-\left(b_{j} I\right)\left(\int_{-1}^{-1+\delta} g(\theta) \operatorname{sen}\left(b_{j} \theta\right) d \theta\right)^{-1} \text { e } \alpha_{0}=\inf \left\{\beta_{j} \mid \beta_{j}>0\right\} .
$$

Então a equação

$$
\lambda=-\alpha I^{-1} \int_{-1}^{-1+\delta} g(\theta) e^{\lambda \theta} d \theta
$$


não tem soluções $\lambda$ com $\Re \lambda \geq 0$ para $0<\alpha<\alpha_{0}$. Se $g(\theta) \equiv 1$ e $0<\delta \leq 1 / 2$, então $\alpha_{0}=-\left(\delta \pi^{2} /(2-\delta)^{2}\right)(2 \cos (\pi / 2-\delta))^{-1}$ e, nesse caso, a equação característica tem precisamente uma solução $\lambda$ com $\Re \lambda>0$ e $0<\Im \lambda<2 \pi$, para $\alpha>\alpha_{0}$.

A equação (2.5), para $g(\theta) \equiv 1$ e $\delta=1$, foi estudada por Oliveira e Carvalho em [11], onde encontramos o seguinte

Teorema 2.1 As raízes imaginárias puras da equação (2.5), com $g(\theta) \equiv 1$ e $\delta=1$, ocorrem para $\alpha=k^{2} \pi^{2} / 2, k$ impar, e são dadas por $\lambda= \pm k \pi i$. Se $0<\alpha<\pi^{2} / 2$, então qualquer raiz $\lambda$ de (2.5) tem parte real negativa, e reciprocamente.

Em [4] Carvalho demonstra o seguinte

Teorema 2.2 A equação (2.5), com $g(\theta) \equiv 1$ e $\delta=1$, tem uma solução 2-periódica não trivial se, e somente se, $\alpha=\pi^{2} / 2$.

Estudando a equação $(2.5) \operatorname{com} g(\theta) \equiv 1$ e $\delta=(n-1) / n$, onde $n=2,3, \ldots$ (note que esses valores de $\delta$ não estão incluídos na segunda parte do Lema 2.1, exceto para $n=2$ ), chegamos ao seguinte

Teorema 2.3 Existem raízes imaginárias puras de $(2.5)$, com $g(\theta) \equiv 1$ e $\delta=(n-$ 1) $/ n, n=2,3, \ldots$, dadas por

$$
\lambda= \pm i n\left(\frac{\pi}{n+1}+k \pi\right), k=0,2, \ldots, \quad \text { e } \quad \lambda= \pm i n\left(-\frac{\pi}{n+1}+k \pi\right), k=2,4, \ldots
$$

e ocorrem respectivamente para

$$
\begin{gathered}
\alpha=\left(n^{2}-n\right)\left[2 \cos \left(\frac{\pi}{n+1}\right)\right]^{-1}\left(\frac{\pi}{n+1}+k \pi\right)^{2}, \quad k=0,2,4, \ldots \\
\text { e } \quad \alpha=\left(n^{2}-n\right)\left[2 \cos \left(\frac{\pi}{n+1}\right)\right]^{-1}\left(-\frac{\pi}{n+1}+k \pi\right)^{2}, \quad k=2,4,6, \ldots
\end{gathered}
$$


Prova Fazendo $\mu=\lambda / n$ e $\beta=\alpha /\left(n^{2}-n\right)$, a equação (2.5) torna-se

$$
\mu^{2}+\beta\left(e^{-\mu}-e^{-n \mu}\right)=0, \quad \mu \neq 0
$$

Seja $\mu=b i$ e podemos considerar $b>0$. Substituindo em (2.6) obtemos:

$$
b^{2}=\beta\left(e^{-i b}-e^{-i n b}\right)=\beta\{[\cos b-\cos (n b)]+i[\operatorname{sen}(n b)-\operatorname{sen} b]\} .
$$

Igualando as partes reais e imaginárias obtemos:

$$
\left\{\begin{array}{l}
b^{2}=\beta[\cos b-\cos (n b)] \\
0=\operatorname{sen}(n b)-\operatorname{sen} b
\end{array}\right.
$$

Da segunda equação temos que $\operatorname{sen} b=\operatorname{sen}(n b)$, ou seja,

$$
\operatorname{sen} b=\operatorname{sen} b\left(\sum_{j=0}^{n-1} \cos ^{j} b \cdot \cos [(n-(j+1)) b]\right) .
$$

Logo, se $\operatorname{sen} b=0$ temos $b=k \pi$, e se sen $b \neq 0$ temos que $b$ é dado pelas soluções da equação

$$
\sum_{j=0}^{n-1} \cos ^{j} b \cdot \cos [(n-(j+1)) b]=1 .
$$

Da primeira equação temos

$$
\beta=b^{2}[\cos b-\cos (n b)]^{-1} \quad \text { se } \quad \cos b-\cos (n b) \neq 0 .
$$

Assim, para $b=k \pi$ temos

$$
(k \pi)^{2}=\beta[\cos (k \pi)-\cos (n k \pi)] \Longrightarrow\left\{\begin{array}{llllll}
(k \pi)^{2}=0, & k & \text { par } & \text { e } & n & \text { qualquer } \\
(k \pi)^{2}=0, & k & \text { ímpar } & \text { e } & n & \text { ímpar } \\
\beta=-(k \pi)^{2} / 2<0, & k & \text { ímpar } & \text { e } & n & \text { par }
\end{array}\right.
$$

que implicam $b=0$ ou $\beta<0$.

Para as soluções de (2.7) obtemos

$$
\beta=\left[2 \cos \left(\frac{\pi}{n+1}\right)\right]^{-1}\left(\frac{\pi}{n+1}+k \pi\right)^{2},
$$


para $b=\pi /(n+1)+k \pi$ e $k=0,2,4, \ldots$

$$
\mathrm{e} \beta=\left[2 \cos \left(\frac{\pi}{n+1}\right)\right]^{-1}\left(-\frac{\pi}{n+1}+k \pi\right)^{2},
$$

para $b=-\pi /(n+1)+k \pi$ e $k=2,4,6, \ldots$

Observe que para $n=2,3, \ldots$

$$
\frac{n \pi}{n+1}=\pi-\frac{\pi}{n+1}, \quad 0<\frac{\pi}{n+1}<\frac{\pi}{2} \quad \text { e } \quad \frac{3 \pi}{2}<-\frac{\pi}{n+1}+2 \pi<2 \pi .
$$

Portanto, as raízes imaginárias puras de (2.5) são

$$
\lambda= \pm i n\left(\frac{\pi}{n+1}+k \pi\right) \quad \text { e } \quad \lambda= \pm i n\left(-\frac{\pi}{n+1}+k \pi\right)
$$

e ocorrem respectivamente para

$$
\begin{gathered}
\alpha=\left(n^{2}-n\right)\left[2 \cos \left(\frac{\pi}{n+1}\right)\right]^{-1}\left(\frac{\pi}{n+1}+k \pi\right)^{2} \text { e } k=0,2, \ldots \\
\text { e } \quad \alpha=\left(n^{2}-n\right)\left[2 \cos \left(\frac{\pi}{n+1}\right)\right]^{-1}\left(-\frac{\pi}{n+1}+k \pi\right)^{2} \text { e } k=2,4, \ldots
\end{gathered}
$$

Observemos que se

$$
g(\theta) \equiv 1 \quad \text { e } \quad \delta=(n-1) / n, \quad n=2,3, \ldots
$$

na equação (2.5), então a constante $\alpha_{0}$ no Lema 2.1 é dada por

$$
\alpha_{0}=\left(n^{2}-n\right)\left[2 \cos \left(\frac{\pi}{n+1}\right)\right]^{-1}\left(\frac{\pi}{n+1}\right)^{2} .
$$

Consideremos agora $C=C([-\delta, 1-\delta], \mathbb{R}), 0<\delta \leq 1 \mathrm{e}$ $K=\left\{\varphi \in C \mid \varphi(t) \leq 0\right.$ se $-\delta \leq t \leq 0, \quad \varphi(0)=0 \quad$ e $\left.\quad \varphi\right|_{[0,1-\delta]} \quad$ não decrescente $\}$.

Para $\varphi \in K \backslash\{0\}$ denote $x(t)=x(t ; \varphi, \alpha)$ a solução da equação $(2.1)$ para $t \geq 1-\delta$ tal que $\left.x\right|_{[-\delta, 1-\delta]}=\varphi$.

Sejam $z_{1}=z_{1}(\varphi)=\inf \{t>1-\delta \mid x(t)=0\}$ e $\psi(t)=x\left(z_{1}+t\right)$ se $t \in[-\delta, 1-\delta]$.

Então 
(i) $\psi(t) \geq 0$ para $t \in[-\delta, 0]$,

(ii) $\psi(0)=0$ e

(iii) $\psi(t)$ é não crescente para $t \in[0,1-\delta]$.

Defina $z_{2}=z_{2}(\varphi)$, o primeiro $t>z_{1}$ tal que $x(t)=0$. Se $\alpha>(1-\delta)^{-1}$, onde $0<\delta \leq 1 / 2$, então $z_{2}<\infty$. Nesse caso, definamos $F_{\alpha}: K \rightarrow C$ por

$$
F_{\alpha}(\varphi)=x\left(z_{2}+t\right) \text { se } \varphi \neq 0 \text { e } t \in[-\delta, 1-\delta] ; \quad F_{\alpha}(0)=0 .
$$

Seja $M_{2}=\sup \left\{\alpha f(x) \mid 0 \leq x \leq M_{1}\right\}$ onde $M_{1}=\alpha B$, e consideremos

$$
K_{1} \stackrel{\text { def }}{=}\left\{\varphi \in K \mid-M_{2} \leq \varphi(t) \leq M_{1} \text { para todo } t\right\} .
$$

Então $K_{1}$ é um conjunto convexo, fechado e limitado de $C$.

Como $f(x) \geq-B$ para todo $x$, temos que $F_{\alpha}\left(K_{1}\right) \subset K_{1}$. Também a aplicação $F_{\alpha}$ é contínua e compacta.

Podemos enunciar o seguinte

Teorema 2.4 Assuma que em (2.1) a função $f$ satisfaça (H.1) e $0<\delta \leq 1 / 2$. Se $\alpha>\alpha_{0}$, onde $\alpha_{0}=-\delta b^{2} /(2 \cos b)$ e $b=\pi /(2-\delta)(\alpha>\pi / 2$ se $\delta=0)$, então a equação (2.1) tem uma solução periódica $x_{\alpha}(t)=x(t)$ tal que $x(0)=0, x(t)>0$ no intervalo $\left(0, z_{1}\right), z_{1}>1-\delta, x(t)<0$ no intervalo $\left(z_{1}, z_{2}\right)$, onde $z_{2}-z_{1}>1-\delta$, e $x\left(t+z_{2}\right)=x(t)$ para todo $t$.

\subsection{Uma equação planar}

Vamos estudar agora as bifurcações de Hopf da equação planar

$$
\dot{x}(t)=F\left(x_{t}\right)
$$

onde $x=\left(x_{1}, x_{2}\right) \in \mathbb{R}^{2}, C=C\left([-r, 0], \mathbb{R}^{2}\right), F: C \rightarrow \mathbb{R}^{2}, F=\left(F_{1}, F_{2}\right)$ e assumindo a hipótese: 


$$
F_{1}(\varphi) \int_{-r}^{-r / 2} \varphi_{2}(\theta) d \theta>0, \quad \text { se } \quad \int_{-r}^{-r / 2} \varphi_{2}(\theta) d \theta \neq 0
$$

(H.2)

$$
F_{2}(\varphi) \int_{-r}^{-r / 2} \varphi_{1}(\theta) d \theta<0, \quad \text { se } \int_{-r}^{-r / 2} \varphi_{1}(\theta) d \theta \neq 0 .
$$

Lema 2.2 Consideremos os funcionais lineares contínuos $T_{1}: C \rightarrow \mathbb{R}$ e $T_{2}: C \rightarrow \mathbb{R}$ dados respectivamente por

$$
T_{1}(\varphi)=\int_{-r}^{-r / 2} \varphi_{1}(\theta) d \theta \text { e } T_{2}(\varphi)=\int_{-r}^{-r / 2} \varphi_{2}(\theta) d \theta
$$

Então $\operatorname{ker} T_{1}$ e $\operatorname{ker} T_{2}$ são subespaços fechados de $C$.

Seja $T: C \rightarrow \mathbb{R}^{2}$ dado por $T(\varphi)=\left(T_{1}(\varphi), T_{2}(\varphi)\right)$. Então

$$
\operatorname{ker} T=\operatorname{ker} T_{1} \cap \operatorname{ker} T_{2}=\left\{\varphi \in C \mid \int_{-r}^{-r / 2} \varphi(\theta) d \theta=0\right\} .
$$

Definição 2.1 Seja $Y=[\gamma, \nu]$ o subespaço de dimensão dois gerado por $\gamma$ e $\nu$, onde $\gamma(\theta)=\left(\gamma_{1}(\theta), \gamma_{2}(\theta)\right)=(1,0)$ e $\nu(\theta)=\left(\nu_{1}(\theta), \nu_{2}(\theta)\right)=(0,1)$, para $\theta \in[-r, 0]$. Por conseguinte, se $Y_{1}=[\gamma]$ e $Y_{2}=[\nu]$, então $Y=Y_{1} \oplus Y_{2}$.

Lema 2.3 Se $C_{0}=\operatorname{ker} T$ e $Y$ é como definido acima, então $C=C_{0} \oplus Y$.

Prova Seja $\varphi=\left(\varphi_{1}, \varphi_{2}\right) \in C$. Então $\varphi=\psi+a \gamma+b \nu=\left(\psi_{1}+a \gamma_{1}, \psi_{2}+b \nu_{2}\right)$ onde

$$
\psi=\left(\psi_{1}, \psi_{2}\right), \quad a=\frac{2}{r} \int_{-r}^{-r / 2} \varphi_{1}(\theta) d \theta \quad \text { e } \quad b=\frac{2}{r} \int_{-r}^{-r / 2} \varphi_{2}(\theta) d \theta .
$$

Temos que

$$
\begin{aligned}
\int_{-r}^{-r / 2} \psi(\theta) d \theta & =\left(\int_{-r}^{-r / 2} \psi_{1}(\theta) d \theta ; \int_{-r}^{-r / 2} \psi_{2}(\theta) d \theta\right)= \\
= & \left(\int_{-r}^{-r / 2} \varphi_{1}(\theta) d \theta-\left(\frac{2}{r} \int_{-r}^{-r / 2} \varphi_{1}(\theta) d \theta\right) \int_{-r}^{-r / 2} \gamma_{1}(\theta) d \theta\right. \\
& \left.\int_{-r}^{-r / 2} \varphi_{2}(\theta) d \theta-\left(\frac{2}{r} \int_{-r}^{-r / 2} \varphi_{2}(\theta) d \theta\right) \int_{-r}^{-r / 2} \nu_{2}(\theta) d \theta\right)=(0,0),
\end{aligned}
$$


ou seja, $\psi \in C_{0}$. Observe que, se $\varphi \in C_{0} \cap Y$, então $\varphi=0$.

Lema 2.4 Se $F$ é contínua, então $F\left(C_{0}\right)=(0,0)$. Em particular, $F(0)=(0,0)$.

Prova Mostremos que $F_{1}\left(C_{0}\right)=0$. Seja $\varphi=\left(\varphi_{1}, \varphi_{2}\right) \in C_{0}$ e considere

$$
\varphi^{k}(\theta)=\left(\varphi_{1}(\theta), \varphi_{2}(\theta)+1 / k\right)
$$

que converge para $\varphi$ quando $k \rightarrow \infty$. Observemos que

$$
\int_{-r}^{-r / 2} \varphi_{2}^{k}(\theta) d \theta=\int_{-r}^{-r / 2} \varphi_{2}(\theta) d \theta+\frac{1}{k} \frac{r}{2}=\frac{r}{2 k}>0 .
$$

Então

$$
F_{1}\left(\varphi^{k}\right)>0 \quad \text { e } \quad F_{1}(\varphi)=\lim _{k \rightarrow \infty} F_{1}\left(\varphi^{k}\right) \geq 0
$$

Consideremos agora

$$
\varphi^{k}(\theta)=\left(\varphi_{1}(\theta), \varphi_{2}(\theta)-1 / k\right)
$$

que também converge para $\varphi$ quando $k \rightarrow \infty$, e observemos que

$$
\int_{-r}^{-r / 2} \varphi_{2}^{k}(\theta) d \theta=\int_{-r}^{-r / 2} \varphi_{2}(\theta) d \theta-\frac{1}{k} \frac{r}{2}=-\frac{r}{2 k}<0 .
$$

$\operatorname{Assim} F_{1}\left(\varphi^{k}\right)<0$ e $F_{1}(\varphi)=\lim _{k \rightarrow \infty} F_{1}\left(\varphi^{k}\right) \leq 0$. Portanto, $F_{1}(\varphi)=0$.

Analogamente, mostramos que $F_{2}\left(C_{0}\right)=0$.

Se $G: C_{0} \oplus Y \rightarrow \mathbb{R}^{2}$ é uma função diferenciável e $\varphi=\varphi^{C_{0}}+\varphi^{Y} \in C_{0} \oplus Y$, denotemos com $D_{1} G(\varphi)$ a derivada parcial de Fréchet de $G$ com respeito a $\varphi^{C_{0}}$ e $D_{2} G(\varphi)$ a derivada parcial de Fréchet de $G$ com respeito a $\varphi^{Y}$.

Lema 2.5 Se $F$ é Fréchet-diferenciável em 0 e $\psi \in C_{0}$, então $D_{1} F_{1}(0) \psi=0$ e $D_{1} F_{2}(0) \psi=0$. 
Prova Pelo lema anterior

$$
\begin{aligned}
& D_{1} F_{1}(0) \psi=d F_{1}(0, \psi)=\lim _{t \rightarrow 0} \frac{F_{1}(0+t \psi)-F_{1}(0)}{t}=\lim _{t \rightarrow 0} \frac{F_{1}(t \psi)}{t}=0 \quad \mathrm{e} \\
& D_{1} F_{2}(0) \psi=d F_{2}(0, \psi)=\lim _{t \rightarrow 0} \frac{F_{2}(0+t \psi)-F_{2}(0)}{t}=\lim _{t \rightarrow 0} \frac{F_{2}(t \psi)}{t}=0 .
\end{aligned}
$$

Vamos verificar agora como $F$ atua em $Y$.

Lema 2.6 Adotando a notação da Definição 2.1, se F é contínua, então $F_{1}(\gamma)=$ $=F_{2}(\nu)=0$.

Prova Sejam $\gamma^{\varepsilon}(\theta)=(1, \varepsilon), \nu^{\varepsilon}(\theta)=(\varepsilon, 1)$ e observemos que

$$
\lim _{\varepsilon \rightarrow 0} \gamma^{\varepsilon}(\theta)=(1,0)=\gamma(\theta) \quad \text { e } \quad \lim _{\varepsilon \rightarrow 0} \nu^{\varepsilon}(\theta)=(0,1)=\nu(\theta) .
$$

Pela hipótese (H.2),

- se $\varepsilon>0$, temos:

$$
F_{1}\left(\gamma^{\varepsilon}\right) \int_{-r}^{-r / 2} \varepsilon d s=F_{1}\left(\gamma^{\varepsilon}\right) \varepsilon \frac{r}{2}>0 \quad \text { e } \quad F_{2}\left(\nu^{\varepsilon}\right) \int_{-r}^{-r / 2} \varepsilon d s=F_{2}\left(\nu^{\varepsilon}\right) \varepsilon \frac{r}{2}<0,
$$

o que implica $F_{1}\left(\gamma^{\varepsilon}\right)>0$ e $F_{2}\left(\nu^{\varepsilon}\right)<0$.

- se $\varepsilon<0$, temos:

$$
F_{1}\left(\gamma^{\varepsilon}\right) \int_{-r}^{-r / 2} \varepsilon d s=F_{1}\left(\gamma^{\varepsilon}\right) \varepsilon \frac{r}{2}>0 \quad \text { e } \quad F_{2}\left(\nu^{\varepsilon}\right) \int_{-r}^{-r / 2} \varepsilon d s=F_{2}\left(\nu^{\varepsilon}\right) \varepsilon \frac{r}{2}<0,
$$

o que implica $F_{1}\left(\gamma^{\varepsilon}\right)<0$ e $F_{2}\left(\nu^{\varepsilon}\right)>0$.

Como $F$ é contínua temos $F_{1}(\gamma)=F_{2}(\nu)=0$. 
Lema 2.7 Se F é Fréchet-diferenciável em 0, então $D_{2} F_{1}(0) \gamma=D_{2} F_{2}(0) \nu=0$.

Prova Pelo lema anterior

$$
\begin{aligned}
& D_{2} F_{1}(0) \gamma=d F_{1}(0, \gamma)=\lim _{t \rightarrow 0} \frac{F_{1}(0+t \gamma)-F_{1}(0)}{t}=\lim _{t \rightarrow 0} \frac{F_{1}(t \gamma)}{t}=0 \quad \mathrm{e} \\
& D_{2} F_{2}(0) \nu=d F_{2}(0, \nu)=\lim _{t \rightarrow 0} \frac{F_{2}(0+t \nu)-F_{2}(0)}{t}=\lim _{t \rightarrow 0} \frac{F_{2}(t \nu)}{t}=0 .
\end{aligned}
$$

Lema 2.8 Se F é Fréchet-diferenciável em 0, então $D_{2} F_{2}(0) \gamma \leq 0$ e $D_{2} F_{1}(0) \nu \geq 0$.

Prova Pela hipótese (H.2) temos

$$
F_{2}(\gamma) \int_{-r}^{-r / 2} \gamma_{1}(s) d s<0 \quad \text { e } \quad F_{1}(\nu) \int_{-r}^{-r / 2} \nu_{2}(s) d s>0
$$

Logo, se $t>0$ teremos

$$
\begin{array}{lll}
F_{2}(t \gamma) t \int_{-r}^{-r / 2} \gamma_{1}(s) d s<0, & \text { o que implica } & F_{2}(t \gamma)<0 \quad \text { e } \\
F_{1}(t \nu) t \int_{-r}^{-r / 2} \nu_{2}(s) d s>0, & \text { o que implica } & F_{1}(t \nu)>0 .
\end{array}
$$

Se $t<0$ teremos

$$
\begin{array}{lll}
F_{2}(t \gamma) t \int_{-r}^{-r / 2} \gamma_{1}(s) d s<0, & \text { o que implica } & F_{2}(t \gamma)>0 \quad \text { e } \\
F_{1}(t \nu) t \int_{-r}^{-r / 2} \nu_{2}(s) d s>0, & \text { o que implica } & F_{1}(t \nu)<0 .
\end{array}
$$

Assim

$$
\begin{aligned}
& D_{2} F_{2}(0) \gamma=\lim _{t \rightarrow 0} \frac{F_{2}(0+t \gamma)-F_{2}(0)}{t}=\lim _{t \rightarrow 0} \frac{F_{2}(t \gamma)}{t} \stackrel{\text { def }}{=} \delta_{2} \leq 0 \quad \mathrm{e} \\
& D_{2} F_{1}(0) \nu=\lim _{t \rightarrow 0} \frac{F_{1}(0+t \nu)-F_{1}(0)}{t}=\lim _{t \rightarrow 0} \frac{F_{1}(t \nu)}{t} \stackrel{\text { def }}{=} \delta_{1} \geq 0 .
\end{aligned}
$$


Observação $2.1 D F(0)(\varphi)=\left(D F_{1}(0) \varphi, D F_{2}(0) \varphi\right)=\left(b \delta_{1}, a \delta_{2}\right)$, onde a e $b$ são dados em (2.9). Consideraremos somente o caso $\delta_{1} \delta_{2}<0$.

A equação linearizada de (2.8) em torno de 0 é

$$
\dot{x}(t)=L x_{t},
$$

onde $L=\left(L_{1}, L_{2}\right)=D F(0)$.

Temos que $L_{j}: C \rightarrow \mathbb{R}, j=1,2$, são funcionais lineares contínuos. Sabemos pelo Teorema de Riesz, que existem funções matriciais $\eta_{j}:[-r, 0] \rightarrow M_{1 \times 2}$ tais que

$$
\eta_{j}(-r)=0 \quad \text { e } \quad L_{j} \varphi=\int_{-r}^{0}\left[d \eta_{j}(\theta)\right] \varphi(\theta), \quad j=1,2 .
$$

Para encontrar a função matricial $\eta_{j}$, usamos o procedimento encontrado em RieszNagy [14], onde estendemos $L_{j}$ a um espaço de Banach contendo $C$ e as funções $\left(\chi_{[-r, \theta]}, 0\right)$ e $\left(0, \chi_{[-r, \theta]}\right)$, onde $\theta \in[-r, 0]$ e $\chi_{[-r, \theta]}$ é a função característica do intervalo $[-r, \theta]$. Então

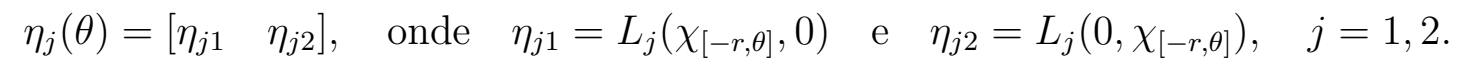

Nosso objetivo agora é representar $L: C \rightarrow \mathbb{R}^{2}$ como $L \varphi=\int_{-r}^{0}[d \eta(\theta)] \varphi(\theta)$. Lembremos que

$$
C=C_{0} \oplus Y, \quad Y=Y_{1} \oplus Y_{2}, \quad \varphi=\varphi^{C_{0}}+\varphi^{Y}, \quad \varphi^{Y}=a \gamma+b \nu,
$$

onde $\quad \gamma=(1,0), \quad \nu=(0,1), \quad a=\frac{2}{r} \int_{-r}^{-r / 2} \varphi_{1}(\theta) d \theta \quad$ e $\quad b=\frac{2}{r} \int_{-r}^{-r / 2} \varphi_{2}(\theta) d \theta$.

Sabemos que $L_{1}: C \rightarrow \mathbb{R}$ satisfaz $L_{1} \gamma=0, L_{1} \nu=\delta_{1}$ e $L_{1}\left(C_{0}\right)=0$. Observemos que para toda $\varphi \in C$ temos

$$
L_{1} \varphi=L_{1}\left(\varphi^{C_{0}}+\varphi^{Y}\right)=L_{1} \varphi^{C_{0}}+L_{1} \varphi^{Y}=a L_{1} \gamma+b L_{1} \nu=b \delta_{1} .
$$

Vamos encontrar agora a matriz $\eta_{1}$ tal que $L_{1} \varphi=\int_{-r}^{0}\left[d \eta_{1}(\theta)\right] \varphi(\theta)$. Seja $\eta_{1}$ matriz $1 \times 2, \eta_{1}(\theta)=\left[\eta_{11}(\theta) \eta_{12}(\theta)\right], \theta \in[-r, 0]$ de variação limitada onde

$$
\eta_{11}(\theta)=L_{1}\left(\chi_{[-r, \theta]}, 0\right) \quad \text { e } \quad \eta_{12}(\theta)=L_{1}\left(0, \chi_{[-r, \theta]}\right) .
$$


Sejam $\omega_{k}$ e $\rho_{k}$ as funções de $C([-r, 0], \mathbb{R})$ definidas respectivamente por

$$
\begin{gathered}
\omega_{k}(s)= \begin{cases}1, & \text { se }-r \leq s \leq \theta-\frac{1}{k}, \\
-k s+k \theta, & \text { se } \theta-\frac{1}{k} \leq s \leq \theta, \\
0, & \text { se } \theta \leq s \leq 0,\end{cases} \\
\rho_{k}(s)= \begin{cases}0, & \text { se }-r \leq s \leq \theta, \\
k s-k \theta, & \text { se } \theta \leq s \leq \theta+\frac{1}{k}, \\
1, & \text { se } \theta+\frac{1}{k} \leq s \leq 0 .\end{cases}
\end{gathered}
$$

Observemos que,

(i) $\gamma=(1,0)=\left(\chi_{[-r, 0]}, 0\right), \quad \nu=(0,1)=\left(0, \chi_{[-r, 0]}\right)$,

(ii) $\left(\rho_{k}, 0\right)=\left(\psi_{k}, 0\right)+a_{k} \gamma \quad$ com $\quad\left(\psi_{k}, 0\right) \in C_{0} \quad$ e $\quad a_{k}=0, \quad$ ou seja, $\rho_{k}=\psi_{k}$.

(iii) $\left(\omega_{k}, 0\right)=\left(\psi_{k}, 0\right)+a_{k} \gamma \operatorname{com}\left(\psi_{k}, 0\right) \in C_{0}$ e $a_{k}=(2 / r)(\theta+r-(1 / 2 k))$.

\section{Então,}

(i) para $\theta \in[-r / 2,0]$, temos que $\chi_{[-r, \theta]}(s)=\chi_{[-r, 0]}(s)-\lim _{k \rightarrow \infty} \rho_{k}(s)$.

Assim, $\left(\chi_{[-r, \theta]}, 0\right)=\lim _{k \rightarrow \infty}\left(\chi_{[-r, 0]}-\rho_{k}, 0\right)=\lim _{k \rightarrow \infty}\left[\left(\chi_{[-r, 0]}, 0\right)-\left(\rho_{k}, 0\right)\right] \mathrm{e}$

$L_{1}\left(\chi_{[-r, \theta]}, 0\right)=\lim _{k \rightarrow \infty}\left[L_{1}\left(\chi_{[-r, 0]}, 0\right)-L_{1}\left(\rho_{k}, 0\right)\right]=L_{1} \gamma-0=0$.

(ii) para $\theta \in[-r,-r / 2], \chi_{[-r, \theta]}(s)=\lim _{k \rightarrow \infty} \omega_{k}(s)$.

Assim,

$$
\begin{gathered}
\left(\chi_{[-r, \theta]}, 0\right)=\lim _{k \rightarrow \infty}\left(\omega_{k}, 0\right)=\lim _{k \rightarrow \infty}\left(\psi_{k}+a_{k} \chi_{[-r, 0]}, 0\right)=\lim _{k \rightarrow \infty}\left[\left(\psi_{k}, 0\right)+\left(a_{k} \chi_{[-r, 0]}, 0\right)\right] \mathrm{e} \\
L_{1}\left(\chi_{[-r, \theta]}, 0\right)=\lim _{k \rightarrow \infty}\left[L_{1}\left(\psi_{k}, 0\right)+a_{k} L_{1}\left(\chi_{[-r, 0]}, 0\right)\right]=0+a_{k} L_{1} \gamma=0 .
\end{gathered}
$$

Assim, $\eta_{11}(\theta)=0$, para $\theta \in[-r, 0]$. 
(iii) para $\theta \in[-r / 2,0]$, temos que

$$
\begin{aligned}
& \left(0, \chi_{[-r, \theta]}\right)=\lim _{k \rightarrow \infty}\left(0, \chi_{[-r, 0]}-\rho_{k}\right)=\lim _{k \rightarrow \infty}\left[\left(0, \chi_{[-r, 0]}\right)-\left(0, \rho_{k}\right)\right] \mathrm{e} \\
& L_{1}\left(0, \chi_{[-r, \theta]}\right)=\lim _{k \rightarrow \infty}\left[L_{1}\left(0, \chi_{[-r, 0]}\right)-L_{1}\left(0, \rho_{k}\right)\right]=L_{1} \nu-0=\delta_{1} .
\end{aligned}
$$

(iv) para $\theta \in[-r,-r / 2]$,

$$
\begin{array}{r}
\left(0, \chi_{[-r, \theta]}\right)=\lim _{k \rightarrow \infty}\left(0, \omega_{k}\right)=\lim _{k \rightarrow \infty}\left(0, \psi_{k}+a_{k} \chi_{[-r, 0]}\right)=\lim _{k \rightarrow \infty}\left[\left(0, \psi_{k}\right)+\left(0, a_{k} \chi_{[-r, 0]}\right)\right] \mathrm{e} \\
L_{1}\left(0, \chi_{[-r, \theta]}\right)=\lim _{k \rightarrow \infty}\left[L_{1}\left(0, \psi_{k}\right)+a_{k} L_{1}\left(0, \chi_{[-r, 0]}\right)\right]=\lim _{k \rightarrow \infty}\left[0+a_{k} L_{1} \nu\right]= \\
=\lim _{k \rightarrow \infty} a_{k} \delta_{1}=\lim _{k \rightarrow \infty} \frac{2}{r}\left(\theta+r-\frac{1}{2 k}\right) \delta_{1}=\frac{2}{r} \delta_{1}(\theta+r) .
\end{array}
$$

Assim,

$$
\eta_{12}(\theta)= \begin{cases}\left(2 \delta_{1} / r\right)(\theta+r), & \text { se } \theta \in[-r,-r / 2] \\ \delta_{1}, & \text { se } \theta \in[-r / 2,0]\end{cases}
$$

Logo,

$$
\begin{aligned}
L_{1} \varphi=\int_{-r}^{0}\left[d \eta_{1}(\theta)\right] \varphi(\theta)=\int_{-r}^{-r / 2}\left[d \eta_{1}(\theta)\right] \varphi(\theta)+\int_{-r / 2}^{0}\left[d \eta_{1}(\theta)\right] \varphi(\theta)= \\
=\int_{-r}^{-r / 2}\left[\begin{array}{ll}
0 & \left.\left(2 \delta_{1} / r\right)\right]\left[\begin{array}{l}
\varphi_{1}(\theta) \\
\varphi_{2}(\theta)
\end{array}\right] d \theta+\int_{-r / 2}^{0}\left[\begin{array}{ll}
0 & 0
\end{array}\right]\left[\begin{array}{l}
\varphi_{1}(\theta) \\
\varphi_{2}(\theta)
\end{array}\right] d \theta= \\
\int_{-r}^{-r / 2}\left(2 \delta_{1} / r\right) \varphi_{2}(\theta) d \theta=\left(2 \delta_{1} / r\right) \int_{-r}^{-r / 2} \varphi_{2}(\theta) d \theta=b \delta_{1} .
\end{array}\right.
\end{aligned}
$$

Procedendo de modo análogo chegamos a

$$
\begin{aligned}
& \eta_{21}(\theta)= \begin{cases}\left(2 \delta_{2} / r\right)(\theta+r), & \text { se } \theta \in[-r,-r / 2], \\
\delta_{2}, & \text { se } \theta \in[-r / 2,0],\end{cases} \\
& \eta_{22}(\theta)=0, \text { para } \theta \in[-r, 0], \quad \text { e } L_{2} \varphi=a \delta_{2} .
\end{aligned}
$$

Portanto, temos 


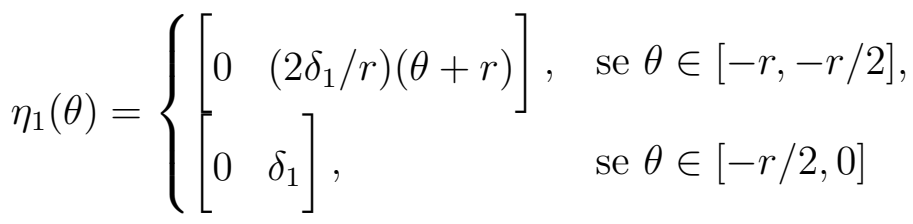

$\mathrm{e}$

$$
\eta_{2}(\theta)= \begin{cases}{\left[\begin{array}{ll}
\left(2 \delta_{2} / r\right)(\theta+r) & 0
\end{array}\right],} & \text { se } \theta \in[-r,-r / 2], \\
{\left[\begin{array}{ll}
\delta_{2} & 0
\end{array}\right],} & \text { se } \theta \in[-r / 2,0] .\end{cases}
$$

Seja $\eta(\theta), \theta \in[-r, 0]$, matriz $2 \times 2$ definida por

$$
\eta(\theta)=\left[\begin{array}{l}
\eta_{1}(\theta) \\
\eta_{2}(\theta)
\end{array}\right] .
$$

Então para toda $\varphi \in C$, temos

$$
\begin{aligned}
L \varphi= & \int_{-r}^{0}[d \eta(\theta)] \varphi(\theta)= \\
& =\int_{-r}^{-r / 2}\left[\begin{array}{cc}
0 & 2 \delta_{1} / r \\
2 \delta_{2} / r & 0
\end{array}\right]\left[\begin{array}{l}
\varphi_{1}(\theta) \\
\varphi_{2}(\theta)
\end{array}\right] d \theta+\int_{-r / 2}^{0}\left[\begin{array}{ll}
0 & 0 \\
0 & 0
\end{array}\right]\left[\begin{array}{l}
\varphi_{1}(\theta) \\
\varphi_{2}(\theta)
\end{array}\right] d \theta= \\
& =\left(\left(2 \delta_{1} / r\right) \int_{-r}^{-r / 2} \varphi_{2}(\theta) d \theta,\left(2 \delta_{2} / r\right) \int_{-r}^{-r / 2} \varphi_{1}(\theta) d \theta\right)=\left(b \delta_{1}, a \delta_{2}\right) .
\end{aligned}
$$

Uma condição necessária e suficiente para a existência de uma solução não trivial da equação linearizada (2.11) da forma $x(t)=e^{\lambda t} u, u \in \mathbb{R}^{2}$, é que $\lambda$ seja raiz da chamada equação característica da parte linear de (2.8) dada por

$$
\operatorname{det} \Delta(\lambda)=0
$$

onde $\Delta(\lambda) \stackrel{\text { def }}{=} \lambda I-L\left(e^{\lambda \cdot} I\right)$ com $I$ a matriz identidade $2 \times 2$. 
Lembrando que $L \varphi=\int_{-r}^{0}[d \eta(\theta)] \varphi(\theta)$ temos

$$
\begin{aligned}
\Delta(\lambda)=\lambda I-L\left(e^{\lambda \cdot} I\right)=\lambda I & -\int_{-r}^{-r / 2}\left[\begin{array}{cc}
0 & 2 \delta_{1} / r \\
2 \delta_{2} / r & 0
\end{array}\right]\left[\begin{array}{cc}
e^{\lambda \theta} & 0 \\
0 & e^{\lambda \theta}
\end{array}\right] d \theta= \\
= & {\left[\begin{array}{cc}
\lambda & \left(2 \delta_{1} / r \lambda\right)\left(e^{-\lambda r / 2}-e^{-\lambda r}\right) \\
\left(2 \delta_{2} / r \lambda\right)\left(e^{-\lambda r / 2}-e^{-\lambda r}\right) & \lambda
\end{array}\right] . }
\end{aligned}
$$

Assim $\operatorname{det} \Delta(\lambda)=0$ implica

$$
\lambda^{2}-4 \delta_{1} \delta_{2}\left(e^{-\lambda r / 2}-e^{-\lambda r}\right)^{2} /\left(r^{2} \lambda^{2}\right)=0 .
$$

Multiplicando a equação (2.15) por $\lambda^{2}$ e fatorando, chegamos a

$$
\left[\lambda^{2}-\frac{2}{r} \sqrt{-\delta_{1} \delta_{2}} i\left(e^{-\lambda r / 2}-e^{-\lambda r}\right)\right]\left[\lambda^{2}+\frac{2}{r} \sqrt{-\delta_{1} \delta_{2}} i\left(e^{-\lambda r / 2}-e^{-\lambda r}\right)\right]=0 .
$$

Fazendo $\alpha \stackrel{\text { def }}{=} 2 \sqrt{-\delta_{1} \delta_{2}} / r, r>0$, temos $\alpha>0$ e

$$
\left[\lambda^{2}-\alpha i\left(e^{-\lambda r / 2}-e^{-\lambda r}\right)\right]\left[\lambda^{2}+\alpha i\left(e^{-\lambda r / 2}-e^{-\lambda r}\right)\right]=0 .
$$

Observação 2.2 (i) $\lambda=0$ é raiz de (2.17) mas não de (2.15).

(ii) $\lambda=$ bi é raiz da equação característica (2.17) se, e somente se, sua conjugada $\bar{\lambda}$ também é.

(iii) A equação (2.17) tem a forma

$$
\left[\lambda-i \alpha \int_{-r}^{-r / 2} e^{\lambda \theta} d \theta\right]\left[\lambda+i \alpha \int_{-r}^{-r / 2} e^{\lambda \theta} d \theta\right]=0 .
$$

(iv) Se $\delta=1 / 2$ em (2.3), a equação (2.18), com $r=1$, pode ser vista como produto de duas equações do tipo (2.4) com parâmetro imaginário puro.

Definindo $\mu=\lambda r / 2$ e $\beta=\left(r^{2} / 4\right) \alpha>0$ a equação (2.17) torna-se 


$$
\left[\mu^{2}-\beta i\left(e^{-\mu}-e^{-2 \mu}\right)\right]\left[\mu^{2}+\beta i\left(e^{-\mu}-e^{-2 \mu}\right)\right]=0, \quad \mu \neq 0 .
$$

Vamos estudar a equação

$$
\mu^{2}-\beta i\left(e^{-\mu}-e^{-2 \mu}\right)=0, \quad \mu \neq 0
$$

A despeito da Observação 2.2 (ii) convém notar que cada fator entre colchetes em (2.17) não é a coeficientes reais e portanto suas raízes não ocorrem aos pares conjugados. O lema a seguir dá uma descrição mais exata deste fato.

Lema 2.9 As raízes imaginárias puras de (2.20) são $\mu=(2 k \pi / 3) i, k=2 \bmod 3$, e elas ocorrem para $\beta=4 \sqrt{3} k^{2} \pi^{2} / 27, k=2 \bmod 3$.

Prova Seja $\mu=b i$. Substituindo em (2.20) obtemos:

$$
-b^{2}=\beta i\left(e^{-b i}-e^{-2 b i}\right)=\beta\{[\operatorname{sen} b-\operatorname{sen}(2 b)]+i[\cos b-\cos (2 b)]\} .
$$

Igualando as partes reais e imaginárias obtemos:

$$
\left\{\begin{array}{cc}
-b^{2} & =\beta[\operatorname{sen} b-\operatorname{sen}(2 b)] \\
0 & =\beta[\cos b-\cos (2 b)]
\end{array}\right.
$$

Da segunda equação em (2.21) temos que $\cos b=\cos (2 b)$, ou seja,

$$
b=2 k \pi \quad \text { ou } \quad b=2 k \pi / 3
$$

onde $k$ é inteiro. Substituindo $b=2 k \pi, k \in \mathbb{Z}$, na primeira equação de (2.21) obtemos $k=0$ e $\beta$ qualquer. Como estamos interessados nas soluções de (2.17), este valor de $b$ não interessa.

Substituindo $b=2 k \pi / 3$ na primeira equação em (2.21) temos:

$$
-(2 k \pi / 3)^{2}=\beta[\operatorname{sen}(2 k \pi / 3)-\operatorname{sen}(4 k \pi / 3)]= \begin{cases}0, & \text { se } k=3 m, m \in \mathbb{Z}, \\ \beta \sqrt{3}, & \text { se } k=1 \bmod 3, \\ -\beta \sqrt{3}, & \text { se } k=2 \bmod 3 .\end{cases}
$$


Portanto as raízes imaginárias puras de $(2.20)$ são $\mu=(2 k \pi / 3) i, k=2 \bmod 3$, e elas ocorrem para $\beta=4 \sqrt{3} k^{2} \pi^{2} / 27, k=2 \bmod 3$.

Afirmação 2.1 Essas raizes são simples.

De fato, se $g(\mu)=\mu^{2}-\beta i\left(e^{-\mu}-e^{-2 \mu}\right)$, temos

$g^{\prime}(2 k \pi i / 3)=(4 k \pi / 3) i+\left(2 \sqrt{3} k^{2} \pi^{2} / 27\right) i-2 k^{2} \pi^{2} / 3 \neq 0, \quad$ onde $\quad k=2 \bmod 3$.

Fazendo $\mu=a+b i$ na equação (2.20) obtemos:

$$
a^{2}-b^{2}+2 a b i=\beta i\left\{e^{-a}[\cos b-i \operatorname{sen} b]-e^{-2 a}[\cos (2 b)-i \operatorname{sen}(2 b)]\right\}
$$

e, igualando partes reais e imaginárias:

$$
\left\{\begin{array}{c}
a^{2}-b^{2}=\beta\left[e^{-a} \operatorname{sen} b-e^{-2 a} \operatorname{sen}(2 b)\right] \\
2 a b=\beta\left[e^{-a} \cos b-e^{-2 a} \cos (2 b)\right] .
\end{array}\right.
$$

Consideremos as equações

$$
\begin{gathered}
H(a, b, \beta) \stackrel{\text { def }}{=} a^{2}-b^{2}-\beta\left[e^{-a} \operatorname{sen} b-e^{-2 a} \operatorname{sen}(2 b)\right]=0 \\
G(a, b, \beta) \stackrel{\text { def }}{=} 2 a b-\beta\left[e^{-a} \cos b-e^{-2 a} \cos (2 b)\right]=0 .
\end{gathered}
$$

Seja $P=\left(0,2 k \pi / 3,4 \sqrt{3} k^{2} \pi^{2} / 27\right), k=2 \bmod 3$. Temos que $H(P)=0$,

$$
H_{a}=2 a-\beta\left[-e^{-a} \operatorname{sen} b+2 e^{-2 a} \operatorname{sen}(2 b)\right], \quad \text { o que implica } \quad H_{a}(P)=-2 k^{2} \pi^{2} / 3,
$$

$H_{b}=-2 b-\beta\left[e^{-a} \cos b-2 e^{-2 a} \cos (2 b)\right], \quad$ o que implica

$$
H_{b}(P)=-(4 k \pi / 3)-\left(2 \sqrt{3} k^{2} \pi^{2}\right) / 27,
$$

e $\quad H_{\beta}=-\left[e^{-a} \operatorname{sen} b-e^{-2 a} \operatorname{sen}(2 b)\right], \quad$ o que implica $\quad H_{\beta}(P)=\sqrt{3}$. 
Temos também que

$$
G(P)=0, \quad G_{a}(P)=4 k \pi / 3+2 \sqrt{3} k^{2} \pi^{2} / 27, \quad G_{b}(P)=-2 k^{2} \pi^{2} / 3 \quad \text { e } \quad G_{\beta}(P)=0 .
$$

Observemos que $H_{a}=G_{b}$ e $G_{a}=-H_{b}$. Assim

$$
\frac{\partial(H, G)}{\partial(a, b)}(P)=\left[H_{a}(P)\right]^{2}+\left[H_{b}(P)\right]^{2}>0
$$

Pelo Teorema da Função Implícita, o sistema $H=0, G=0$, define para cada $k=2 \bmod 3$ uma única curva

$$
\Gamma_{k}:\left\{\begin{array}{l}
a=a(\beta) \\
b=b(\beta)
\end{array}\right.
$$

em torno do ponto $\beta_{k}=4 \sqrt{3} k^{2} \pi^{2} / 27$ tal que $a\left(\beta_{k}\right)=0$ e $b\left(\beta_{k}\right)=2 k \pi / 3$.

Assim, teremos

$$
\left\{\begin{array}{l}
H(a(\beta), b(\beta), \beta)=0 \\
G(a(\beta), b(\beta), \beta)=0
\end{array}\right.
$$

donde

$$
\left\{\begin{array}{l}
H_{a} a^{\prime}(\beta)+H_{b} b^{\prime}(\beta)+H_{\beta}=0 \\
G_{a} a^{\prime}(\beta)+G_{b} b^{\prime}(\beta)+G_{\beta}=0 .
\end{array}\right.
$$

Lembrando que $G_{a}(P)=-H_{b}(P)$ e $G_{b}(P)=H_{a}(P)$, teremos

$$
\left\{\begin{aligned}
H_{a}(P) a^{\prime}\left(\beta_{k}\right)+H_{b}(P) b^{\prime}\left(\beta_{k}\right) & =-\sqrt{3} \\
-H_{b}(P) a^{\prime}\left(\beta_{k}\right)+H_{a}(P) b^{\prime}\left(\beta_{k}\right) & =0 .
\end{aligned}\right.
$$

Resolvendo o sistema vemos que

$$
a^{\prime}\left(\beta_{k}\right)=\frac{2 \sqrt{3} \pi^{2} k^{2}}{3\left(H_{a}^{2}+H_{b}^{2}\right)}>0 .
$$

Como $\beta=\left(r^{2} / 4\right) \alpha$, temos que $\beta_{k}=\beta_{k}\left(\alpha_{k}\right)$ e

$$
a^{\prime}\left(\alpha_{k}\right)=\left(r^{2} / 4\right) a^{\prime}\left(\beta_{k}\right)>0 .
$$


Também, da segunda equação do sistema (2.25) temos $a^{\prime}\left(\beta_{k}\right) / b^{\prime}\left(\beta_{k}\right)=H_{a}(P) / H_{b}(P)$. Como

$$
\frac{d a}{d b}(P)=\frac{a^{\prime}\left(\beta_{k}\right)}{b^{\prime}\left(\beta_{k}\right)}=\frac{9 \pi k}{18+\sqrt{3} \pi k} \quad \text { e } \quad k=2 \bmod 3
$$

temos

$$
\frac{d a}{d b}(P)>0 \quad \text { para } \quad k \neq-1 \quad \text { e } \quad \frac{d a}{d b}(P)<0 \quad \text { para } \quad k=-1 .
$$

Para a equação

$$
\lambda^{2}+\alpha i\left(e^{-\lambda r / 2}-e^{-\lambda r}\right)=0
$$

corespondente ao segundo fator de (2.17), fazendo a mesma mudança de variáveis, obtemos a seguinte equação

$$
\mu^{2}+\beta i\left(e^{-\mu}-e^{-2 \mu}\right)=0, \quad \mu \neq 0
$$

e com procedimento análogo obtemos os seguintes resultados

Lema 2.10 As raízes imaginárias puras de (2.27) são $\mu=(2 k \pi / 3) i, k=1 \bmod 3$ e elas ocorrem para $\beta=4 \sqrt{3} k^{2} \pi^{2} / 27, k=1 \bmod 3$. Além disso, essas raízes são simples.

Fazendo $\mu=a+i b$ na equação (2.27) obtemos:

$$
a^{2}-b^{2}+2 a b i=-\beta i\left\{e^{-a}[\cos b-i \operatorname{sen} b]-e^{-2 a}[\cos (2 b)-i \operatorname{sen}(2 b)]\right\} .
$$

Igualando partes reais e imaginárias obtemos:

$$
\left\{\begin{array}{c}
a^{2}-b^{2}=-\beta\left[e^{-a} \operatorname{sen} b-e^{-2 a} \operatorname{sen}(2 b)\right] \\
2 a b=-\beta\left[e^{-a} \cos b-e^{-2 a} \cos (2 b)\right] .
\end{array}\right.
$$

Consideremos as equações

$$
\begin{aligned}
& H(a, b, \beta) \stackrel{\text { def }}{=} a^{2}-b^{2}+\beta\left[e^{-a} \operatorname{sen} b-e^{-2 a} \operatorname{sen}(2 b)\right]=0 \\
& G(a, b, \beta) \stackrel{\text { def }}{=} 2 a b+\beta\left[e^{-a} \cos b-e^{-2 a} \cos (2 b)\right]=0 .
\end{aligned}
$$


Seja $P^{*}=\left(0,2 k \pi / 3,4 \sqrt{3} k^{2} \pi^{2} / 27\right), k=1 \bmod 3$. Temos que $H\left(P^{*}\right)=0$,

$$
H_{a}\left(P^{*}\right)=-2 k^{2} \pi^{2} / 3, \quad H_{b}\left(P^{*}\right)=-4 k \pi / 3+2 \sqrt{3} k^{2} \pi^{2} / 27 \quad \text { e } \quad H_{\beta}\left(P^{*}\right)=\sqrt{3} .
$$

Temos também que

$$
\begin{gathered}
G_{a}\left(P^{*}\right)=4 k \pi / 3-2 \sqrt{3} k^{2} \pi^{2} / 27, \quad G_{b}\left(P^{*}\right)=-2 k^{2} \pi^{2} / 3 \quad G_{\beta}\left(P^{*}\right)=0 \quad \text { e } \\
\frac{\partial(H, G)}{\partial(a, b)}\left(P^{*}\right)=\left[H_{a}\left(P^{*}\right)\right]^{2}+\left[H_{b}\left(P^{*}\right)\right]^{2}>0 .
\end{gathered}
$$

Pelo Teorema da Função Implícita, o sistema $H=0, G=0$, define para cada $k=1 \bmod 3$ uma única curva

$$
\Gamma_{k}:\left\{\begin{array}{l}
a=a(\beta) \\
b=b(\beta)
\end{array}\right.
$$

em torno do ponto $\beta_{k}=4 \sqrt{3} \pi^{2} k^{2} / 27$ tal que $a\left(\beta_{k}\right)=0, b\left(\beta_{k}\right)=2 k \pi / 3$.

Lembrando que $G_{a}\left(P^{*}\right)=-H_{b}\left(P^{*}\right)$ e $G_{b}\left(P^{*}\right)=H_{a}\left(P^{*}\right)$ teremos

$$
\left\{\begin{aligned}
H_{a}\left(P^{*}\right) a^{\prime}\left(\beta_{k}\right)+H_{b}\left(P^{*}\right) b^{\prime}\left(\beta_{k}\right) & =-\sqrt{3} \\
-H_{b}\left(P^{*}\right) a^{\prime}\left(\beta_{k}\right)+H_{a}\left(P^{*}\right) b^{\prime}\left(\beta_{k}\right) & =0
\end{aligned}\right.
$$

Resolvendo o sistema vemos que $a^{\prime}\left(\beta_{k}\right)>0$ e, portanto, $a^{\prime}\left(\alpha_{k}\right)>0$.

Também, $d a / d b=-9 \pi k /(-18+\sqrt{3} k \pi)$. Como $k=1 \bmod 3$

$$
\frac{d a}{d b}\left(P^{*}\right)<0 \quad \text { para } \quad k \neq 1 \quad \text { e } \quad \frac{d a}{d b}\left(P^{*}\right)>0, \quad \text { para } \quad k=1 .
$$

Como observamos anteriormente, as raízes de (2.20) e (2.27) não ocorrem aos pares conjugados.

A discussão acima permite enunciar o seguinte lema sobre a localização das raízes imaginárias puras da equação (2.17).

Lema 2.11 As raízes imaginárias puras da equação (2.17) são

$$
\lambda= \pm(4 k \pi / 3 r) i, \quad k \in \mathbb{Z}_{+}, \quad k \neq 3 m, m \in \mathbb{Z}_{+},
$$

e ocorrem para $\alpha=16 \sqrt{3} \pi^{2} k^{2} / 27 r^{2}, k \in \mathbb{Z}_{+}, k \neq 3 m, m \in \mathbb{Z}_{+}$. 
Mantendo a definição do parâmetro $\alpha=(2 / r) \sqrt{-\delta_{1} \delta_{2}}$, podemos resumir as considerações acima no seguinte teorema, que é uma conseqüência de [5, Th.1.1,p. 246].

Teorema 2.5 Se (H.2) é satisfeita, então existe uma seqüência $\left(\alpha_{k}\right)$,

$$
\alpha_{k}=\frac{16 \sqrt{3} \pi^{2} k^{2}}{27 r^{2}}, \quad k \in \mathbb{Z}_{+}, \quad k \neq 3 m, \quad m \in \mathbb{Z}_{+},
$$

tal que a equação (2.8) tem uma bifurcação de Hopf em $\alpha=\alpha_{k}$.

Prova A prova segue dos resultados desse capítulo.

Exemplo 2.1 Seja

$$
\dot{x}(t)=F\left(x_{t}\right)
$$

onde

$$
F(\varphi)=\left(\int_{-r}^{-r / 2} h_{2}\left(\varphi_{2}(\theta)\right) d \theta,-\int_{-r}^{-r / 2} h_{1}\left(\varphi_{1}(\theta)\right) d \theta\right),
$$

$\varphi=\left(\varphi_{1}, \varphi_{2}\right), r>0$ e $h_{j}: \mathbb{R} \rightarrow \mathbb{R}, j=1,2$, são funções contínuas tais que

(i) $z h_{j}(z)>0$ se $z \neq 0, j=1,2$,

(ii) $h_{j}^{\prime}(0) \stackrel{\text { def }}{=} \varrho_{j}>0, j=1,2$,

(iii) e $F$ satisfaz a hipótese $(\mathbf{H . 2})$.

Com a notação anterior temos

$$
D_{2} F_{2}(0) \gamma=-\frac{r}{2} \varrho_{1}=\delta_{2}<0 \quad \text { e } \quad D_{2} F_{1}(0) \nu=\frac{r}{2} \varrho_{2}=\delta_{1}>0
$$

e a linearização de $F$ em torno da origem é dada por

$$
\begin{aligned}
L \varphi=\left(\frac{2}{r} \int_{-r}^{-r / 2} \varphi_{2}(\theta) d \theta\left(\frac{r}{2} \varrho_{2}\right)\right. & \left., \frac{2}{r} \int_{-r}^{-r / 2} \varphi_{1}(\theta) d \theta\left(-\frac{r}{2} \varrho_{1}\right)\right)= \\
& =\left(\varrho_{2} \int_{-r}^{-r / 2} \varphi_{2}(\theta) d \theta,-\varrho_{1} \int_{-r}^{-r / 2} \varphi_{1}(\theta) d \theta\right) .
\end{aligned}
$$


Também $L \varphi$ tem a representação matricial $L \varphi=\int_{-r}^{0}[d \eta(\theta)] \varphi(\theta)$ onde

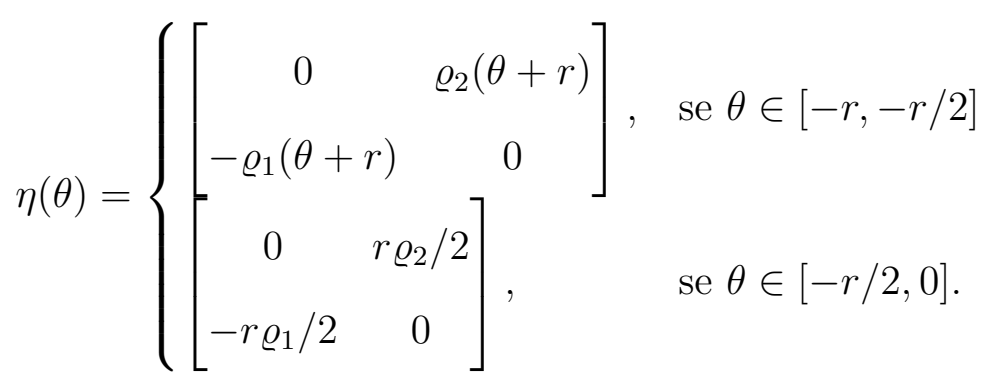

A equação característica da parte linear de (2.29) é dada por

$$
\lambda^{4}+\varrho_{1} \varrho_{2}\left(e^{-\lambda r / 2}-e^{-\lambda r}\right)^{2}=0
$$

e suas raízes imaginárias puras são

$$
\lambda= \pm(4 k \pi / 3 r) i, \quad k \in \mathbb{Z}_{+}, \quad k \neq 3 m, \quad m \in Z_{+},
$$

e ocorrem para $\sqrt{\varrho_{1} \varrho_{2}}=16 \sqrt{3} \pi^{2} k^{2} / 27 r^{2}, k \in \mathbb{Z}_{+}, k \neq 3 m, m \in Z_{+}$, valores nos quais ocorrem bifurcações de Hopf. 


\section{Capítulo 3}

\section{Soluções periódicas de um sistema impulsivo}

\subsection{Introdução}

A principal motivação deste capítulo é o exemplo do final do capítulo anterior. Na verdade, grande parte de nossa investigação será dedicada àquele caso especial. Como já observamos anteriormente, a versão escalar desse exemplo foi exaustivamente estudada por vários autores, veja Nussbaum [9] e Walther [15], por exemplo.

Provaremos a existência de soluções periódicas não triviais do problema de auto-sustentação

$$
\begin{gathered}
\dot{x}(t)=F\left(x_{t}\right) \\
x(t)=\left(0, x_{2}(t)\right), x_{2}(t) \geq c \quad \text { implica } \quad x\left(t^{+}\right)=\left(0, x_{2}(t)-\gamma\left(x_{2}(t)-c\right)\right)
\end{gathered}
$$

onde $c>0, \gamma>0, x=\left(x_{1}, x_{2}\right) \in \mathbb{R}^{2}, F: C\left([-1,0], \mathbb{R}^{2}\right) \rightarrow \mathbb{R}^{2}, F(\varphi)=\left(F_{1}(\varphi), F_{2}(\varphi)\right)$,

$$
F(\varphi)=\left(\int_{-1}^{-1 / 2} h_{2}\left(\varphi_{2}(\theta)\right) d \theta,-\int_{-1}^{-1 / 2} h_{1}\left(\varphi_{1}(\theta)\right) d \theta\right)
$$

$\varphi=\left(\varphi_{1}, \varphi_{2}\right), h_{j}: \mathbb{R} \rightarrow \mathbb{R}$, funções contínuas tais que $z h_{j}(z)>0, z \neq 0, j=1,2$. 
Isso é feito mostrando que um certo operador de retorno definido pelo fluxo num cone do espaço de fase $C\left([-1,0], \mathbb{R}^{2}\right)$, tem um ponto fixo não trivial.

A maior dificuldade desse procedimento se deve ao fato de que a origem é um ponto fixo, conhecido a priori. Essa dificuldade é contornada usando um teorema que depende do conceito de ejetividade e garante a existência de pontos fixos não nulos.

Observemos que para convenientes funções $h_{j}, j=1,2$, a função $F$ satisfaz a hipótese (H.2) do capítulo anterior.

Definição 3.1 Seja $X$ um espaço de Banach, $U$ um subconjunto de $X$ e $x_{0} \in U$. Dada uma aplicação $A: U \backslash\left\{x_{0}\right\} \rightarrow X$, o ponto $x_{0} \in U$ é dito um ponto ejetivo de $A$ se existe uma vizinhança aberta $G \subset X$ de $x_{0}$ tal que para todo $y \in G \cap U, y \neq x_{0}$, existe um inteiro $m=m(y)$ tal que $A^{m} y \notin G \cap U$.

Notação Para qualquer $M>0$, denotamos

$$
S_{M}=\{x \in X|| x \mid=M\} \text { e } B_{M}=\{x \in X|| x \mid<M\}
$$

O teorema seguinte é devido a Nussbaum, veja por exemplo [5, Th. 2.1, p. 249].

Teorema 3.1 Se K é um conjunto fechado, limitado, convexo, de dimensão infinita em um espaço de Banach $X, A: K \backslash\left\{x_{0}\right\} \rightarrow K$ uma aplicação completamente contínua e $x_{0} \in K$ é um ponto ejetivo de $A$, então existe um ponto fixo de $A$ em $K \backslash\left\{x_{0}\right\}$.

Observação 3.1 Em nosso caso é suficiente entender que $K \subset X$ é um conjunto de dimensão infinita se não estiver contido em qualquer $V \subset X$ subespaço linear afim de dimensão finita, veja [9].

O subconjunto de $C=C\left([-1,0], \mathbb{R}^{2}\right)$, dado por

$$
\begin{aligned}
& K=\{\varphi \in C \mid \varphi(\theta) \in Q_{1},-1 \leq \theta \leq 0, \varphi_{1}(-1)=0, \varphi_{2}(-1) \geq 0 \\
&\left.\varphi_{1} \text { não decrescente em }[-1,0] \text { e } \varphi_{2} \text { não decrescente em }[-1,-1 / 2]\right\}
\end{aligned}
$$


é de grande importância neste capítulo. Observemos que $K$ é um cone, isto é, $K$ é um subconjunto fechado e convexo do espaço de Banach $C, \lambda K \subset K$ se $\lambda \geq 0$ e $K \cap(-K)=\{0\}$.

Daqui em diante indicamos por $Q_{1}, Q_{2}, Q_{3}$ e $Q_{4}$ os seguintes quadrantes do plano $x_{1} x_{2}$ :

$$
\begin{aligned}
& Q_{1} \stackrel{\text { def }}{=}\left\{\left(x_{1}, x_{2}\right) \in \mathbb{R}^{2} \mid x_{1} \geq 0, x_{2} \geq 0\right\}, \\
& Q_{2} \stackrel{\text { def }}{=}\left\{\left(x_{1}, x_{2}\right) \in \mathbb{R}^{2} \mid x_{1} \geq 0, x_{2} \leq 0\right\}, \\
& Q_{3} \stackrel{\text { def }}{=}\left\{\left(x_{1}, x_{2}\right) \in \mathbb{R}^{2} \mid x_{1} \leq 0, x_{2} \leq 0\right\}, \\
& Q_{4} \stackrel{\text { def }}{=}\left\{\left(x_{1}, x_{2}\right) \in \mathbb{R}^{2} \mid x_{1} \leq 0, x_{2} \geq 0\right\} .
\end{aligned}
$$

Definição 3.2 Dizemos que $x$ é solução periódica lentamente espiralante de (3.1) se existem $t_{0}, t_{1}, t_{2}, t_{3}, t_{4}$, tais que $t_{i}-t_{i-1}>1, i=1,2,3,4, x(t) \in Q_{i}, t_{i-1} \leq t \leq t_{i}$, $i=1,2,3,4$, e $x_{t_{4}}=x_{t_{0}}$.

\subsection{Soluções lentamente espiralantes}

Nosso objetivo nessa seção é investigar a existência de soluções periódicas lentamente espiralantes do sistema (3.1),(3.2).

Dada $\varphi \in K$, seja $x(\cdot ; \varphi)$ a solução de $(3.1)$ definida em $[-1, \infty)$ tal que $\left.x\right|_{[-1,0]}=\varphi$. Quando não houver dúvida quanto a condição inicial $\varphi$, usaremos a notação $x(t)$.

É conveniente termos a equação (3.1) também na forma

$$
\left\{\begin{array}{l}
\dot{x_{1}}(t)=\int_{-1}^{-1 / 2} h_{2}\left(x_{2}(t+\theta)\right) d \theta \\
\dot{x_{2}}(t)=-\int_{-1}^{-1 / 2} h_{1}\left(x_{1}(t+\theta)\right) d \theta
\end{array}\right.
$$

ou ainda,

$$
\left\{\begin{array}{l}
\dot{x}_{1}(t)=\int_{t-1}^{t-1 / 2} h_{2}\left(x_{2}(s)\right) d s \\
\dot{x_{2}}(t)=-\int_{t-1}^{t-1 / 2} h_{1}\left(x_{1}(s)\right) d s .
\end{array}\right.
$$


Provamos a seguir alguns lemas que permitem definir o operador de retorno citado na Introdução.

Lema 3.1 Se F é dada em (3.3) com $h_{1}$ não decrescente, então existe uma função

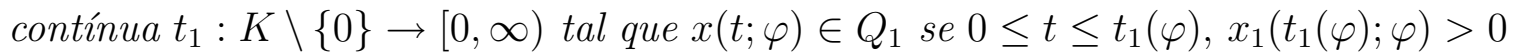
e $x(t ; \varphi) \in \operatorname{int}\left(Q_{2}\right)$ se $t_{1}(\varphi)<t<t_{1}(\varphi)+\delta$ para algum $\delta>0$.

Prova Se $\varphi_{2}(0)=0$ existem três casos a considerar:

(i) $\varphi_{1} \neq 0$ em $[-1,-1 / 2]$. Assim, $\dot{x}_{2}(0)=-\int_{-1}^{-\frac{1}{2}} h_{1}\left(x_{1}(\theta)\right) d \theta<0$ e $\dot{x}_{2}(t)<0$, $0 \leq t<t+\delta$, para algum $\delta>0$. Neste caso tomamos $t_{1}(\varphi)=0$.

(ii) $\varphi_{1}=0$ em $\left[-1,-\frac{1}{2}\right]$ e $\varphi_{1} \neq 0$ em $\left[-\frac{1}{2}, 0\right]$, então $\dot{x}_{2}(0)=0$ e $\dot{x}_{2}(t) \leq 0$ para $0<t \leq \frac{1}{2}$. Se $\dot{x}_{2}(t)<0$ para $0<t \leq \frac{1}{2}$ tomamos $t_{1}(\varphi)=0$. Se $\dot{x}_{2}(t)=0$, $0 \leq t \leq \delta$, para algum $\delta>0$ e $\dot{x}_{2}(t)<0$ para $\delta<t \leq \frac{1}{2}$ tomamos $t_{1}(\varphi)=\delta$.

(iii) $\varphi_{1}=0$ em $[-1,0]$, então $\dot{x}_{2}(t)=0$ para $0 \leq t \leq \frac{1}{2}$, o que implica $x_{2}(t)=0$ para $0 \leq t \leq \frac{1}{2}$. Neste caso, existe um subintervalo de $[-1,0]$ onde $\varphi_{2}>0$. Portanto $x_{1}(t)>0$ em um subintervalo de $[0,1 / 2]$ e existe um primeiro instante $t_{1}(\varphi)>0$ tal que $x_{2}(t)<0$ para $t_{1}(\varphi)<t<t_{1}(\varphi)+\delta$, para algum $\delta>0$.

Consideremos agora $\varphi_{2}(0)>0$.

Afirmação: Existe $\hat{t}=\hat{t}(\varphi)>0$ tal que $x(\hat{t})=x(\hat{t} ; \varphi) \in Q_{2}$.

De fato, suponhamos por contradição que exista uma função $\varphi \in K \backslash\{0\}$ tal que $x(t) \in Q_{1}, t \in[0, \infty)$.

Observemos que existe $\bar{t} \in[0, \infty)$ tal que $x_{1}(\bar{t})>0$. De fato,

(i) Se $\varphi_{1} \not \equiv 0$, então existe $\bar{\theta},-1 \leq \bar{\theta} \leq 0$ tal que $\varphi_{1}(\bar{\theta})>0$, e neste caso podemos tomar $\bar{t}=0$ já que $\varphi_{1}$ é crescente.

(ii) Se $\varphi_{1} \equiv 0$, suponha por contradição, que não exista $\bar{t} \in[0, \infty)$ tal que $x_{1}(\bar{t})>0$. Então $x_{1}(t)=0$, para $t \in[-1, \infty)$, o que implica $\dot{x_{1}}(t)=0$, para todo $t \geq-1$. Por outro lado, como $\varphi \in K \backslash\{0\}$, existe $\bar{\theta} \in[-1,0]$ tal que $\varphi_{2}(\bar{\theta})>0$. Como 
$\varphi_{2}$ é contínua, existe uma vizinhança $V$ de $\bar{\theta}$ tal que $\varphi_{2}(\theta)>0$, para todo $\theta \in V$. Como $z h_{2}(z)>0, z \neq 0$, temos que $h_{2}\left(\varphi_{2}(\theta)\right)>0$, para todo $\theta \in V$. Então existe $\tau \geq 0$ tal que $\dot{x_{1}}(\tau)=\int_{\tau-1}^{\tau-1 / 2} h_{2}\left(x_{2}(\theta)\right) d \theta>0$, o que é uma contradição, ou seja, existe $\bar{t} \in[0, \infty)$ tal que $x_{1}(\bar{t})>0$.

Se não existisse $\hat{t}$, teríamos $x(t) \in Q_{1}$ sempre. Portanto $h_{2}\left(x_{2}(s)\right) \geq 0$, o que implica $x_{1}$ não decrescente. Assim $x_{1}(t) \geq x_{1}(\bar{t})>0$, para $t \in[\bar{t}, \infty)$. Como $h_{1}$ é não decrescente, temos $h_{1}\left(x_{1}(\bar{t})\right) \leq h_{1}\left(x_{1}(t)\right)$, para todo $t \geq \bar{t}$, e como $z h_{1}(z)>0, z \neq 0$,

$$
\dot{x}_{2}(t)=-\int_{t-1}^{t-1 / 2} h_{1}\left(x_{1}(s)\right) d s \leq-(1 / 2) h_{1}\left(x_{1}(\bar{t})\right)<0, \quad t \geq \bar{t}+1,
$$

o que prova nossa afirmação.

Deste modo, existe $t_{1}=t_{1}(\varphi)$ tal que $x(t) \in Q_{1}, 0 \leq t \leq t_{1}$, e $x(t) \in \operatorname{int}\left(Q_{2}\right)$, $t_{1}<t<t_{1}+\delta$, para algum $\delta>0$.

A continuidade de $t_{1}$ segue do teorema da continuidade em relação às condições iniciais.

Mantendo as notações do Lema 3.1, se $\widetilde{t}=t_{1}+1$ e $\psi=x_{\tilde{t}}$, segue do sistema (3.6) que $\psi_{2}(\theta)$ é não crescente no intervalo $[-1,0]$ e $\psi_{1}(\theta)$ é não decrescente em $[-1,-1 / 2]$. Assim, se $\Psi$ é a rotação dada por $\left[\begin{array}{rr}0 & 1 \\ -1 & 0\end{array}\right]$ temos que

1. existe $\theta_{2} \in(-1,0]$ tal que $\psi_{2}\left(\theta_{2}\right)<0$ e $\psi_{1}\left(\theta_{2}\right)=0$ ou

2. $\psi \in \Psi K \backslash\{0\}$.

Se $\psi \in \Psi K \backslash\{0\}$ e não existe $\theta_{2} \in(-1,0]$ tal que $\psi_{2}\left(\theta_{2}\right)<0$ e $\psi_{1}\left(\theta_{2}\right)=0$, argumentos análogos aos do Lema 3.1, supondo $h_{2}$ não decrescente, garantem a existência de $t_{2}=t_{2}(\varphi), t_{2}>\tilde{t}$ tal que $x(t) \in Q_{2}, t_{1} \leq t \leq t_{2}$ e $x(t) \in \operatorname{int}\left(Q_{3}\right)$ para $t_{2}<t<t_{2}+\delta$, para algum $\delta>0$. 
Portanto, valendo qualquer das alternativas acima, fica estabelecida a existência de $t_{2}=t_{2}(\varphi)$ tal que $x(t)$ cruza o semi eixo negativo $x_{2}$ no instante $t=t_{2}$.

Argumentando assim sucessivamente, prova-se que a solução $x(t)$ gira no sentido horário em torno da origem, de modo que existem instantes $0<t_{1}<t_{2}<t_{3}<t_{4}<t_{5}$ tais que $x\left(t_{1}\right), x\left(t_{3}\right)$ e $x\left(t_{5}\right)$ estão no eixo $x_{1}$ e $x\left(t_{2}\right)$ e $x\left(t_{4}\right)$ estão no eixo $x_{2}$, como sugere a Figura 3.1.

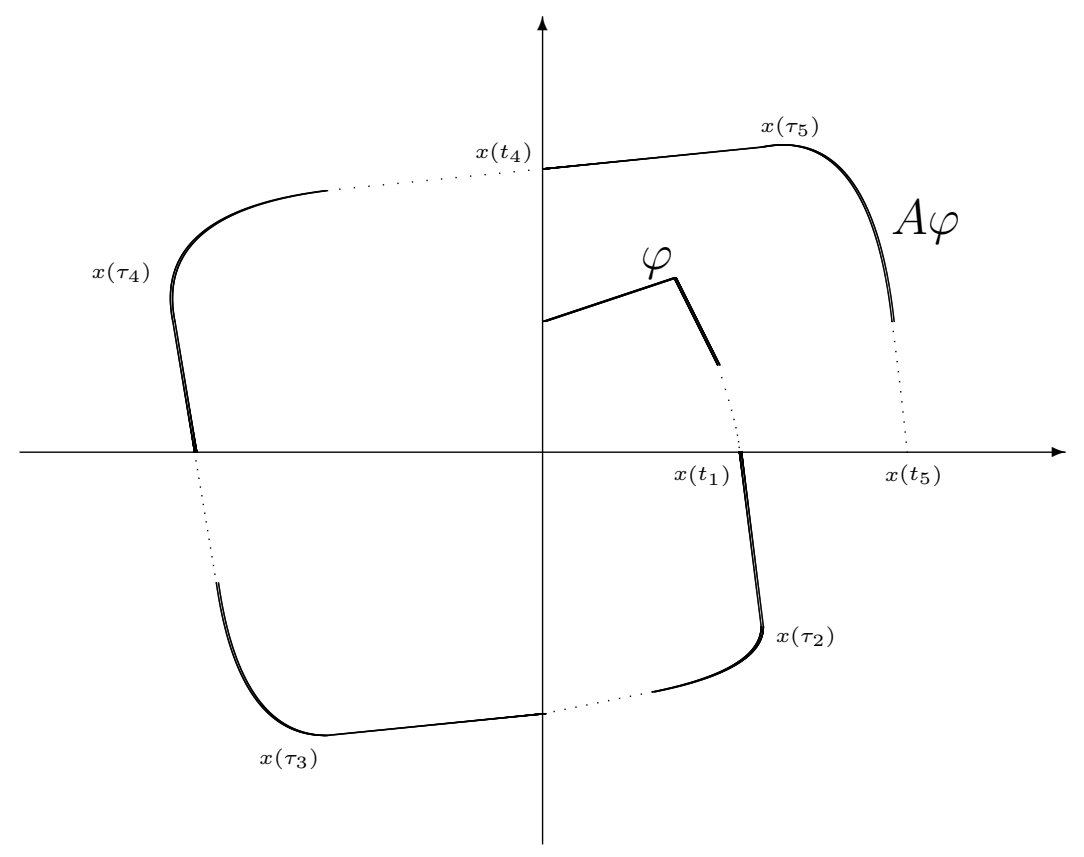

Figura 3.1: O operador de retorno e instantes $t_{j}$ e $\tau_{j}$

É também uma conseqüência da continuidade com relação às condições iniciais que as funções $t_{j}: K \backslash\{0\} \rightarrow[0, \infty), j=2,3,4,5$, são contínuas.

Definição 3.3 Definimos $\tau_{i+1}(\varphi) \in\left(t_{i}(\varphi), t_{i+1}(\varphi)\right)$ os instantes onde $x_{1}$ ou $x_{2}$ assume seu extremo, conforme seja $i=1,3$ ou $i=2,4$, respectivamente, para cada $\varphi \in K \backslash\{0\}$. 
O lema seguinte e seu corolário garantem que o operador $A: K \rightarrow C$ dado por

$$
A \varphi=x_{\tau(\varphi)}(\cdot ; \varphi), \quad \text { se } \quad \varphi \in K \backslash\{0\} ; \quad A 0=0,
$$

onde $\tau(\varphi)=t_{4}(\varphi)+1$, satisfaz $A K \subset K$.

Lema 3.2 Suponhamos no sistema (3.6) que as funções $h_{j}, j=1,2$, sejam não decrescentes e satisfaçam a desigualdade $\left|h_{j}(z)\right| \leq \sqrt{2}|z|$. Então $t_{5}-\tau_{5} \geq 1$.

Prova De acordo com o sistema (3.6) e a escolha de $\tau_{5}$, temos

$$
\dot{x_{2}}\left(\tau_{5}\right)=0 \quad \text { e } \quad 1 / 2<\tau_{5}-t_{4}<1 .
$$

Aplicando o Teorema do Valor Médio a $x_{2}$ no intervalo $\left[\tau_{5}, t_{5}\right]$ temos que existe $t^{\prime} \in\left(\tau_{5}, t_{5}\right)$ tal que

$$
x_{2}\left(t_{5}\right)-x_{2}\left(\tau_{5}\right)=\dot{x_{2}}\left(t^{\prime}\right)\left(t_{5}-\tau_{5}\right)=-\int_{t^{\prime}-1}^{t^{\prime}-1 / 2} h_{1}\left(x_{1}(s)\right) d s\left(t_{5}-\tau_{5}\right) .
$$

Portanto,

$$
x_{2}\left(\tau_{5}\right) \leq \sqrt{2} \int_{t^{\prime}-1}^{t^{\prime}-1 / 2} x_{1}(s) d s\left(t_{5}-\tau_{5}\right) \leq \frac{\sqrt{2}}{2} x_{1}\left(t_{5}\right)\left(t_{5}-\tau_{5}\right) .
$$

Aplicando o Teorema do Valor Médio a $x_{1}$ no intervalo $\left[t_{4}, t_{5}\right]$ temos que existe $t^{\prime \prime} \in\left(t_{4}, t_{5}\right)$ tal que

$$
x_{1}\left(t_{5}\right)=\int_{t^{\prime \prime}-1}^{t^{\prime \prime}-1 / 2} h_{2}\left(x_{2}(s)\right) d s\left(t_{5}-t_{4}\right) \leq \frac{\sqrt{2}}{2} x_{2}\left(\tau_{5}\right)\left(t_{5}-t_{4}\right) .
$$

Assim, combinando esta desigualdade com (3.8),

$$
2 x_{1}\left(t_{5}\right) \leq \sqrt{2} x_{2}\left(\tau_{5}\right)\left(t_{5}-t_{4}\right) \leq x_{1}\left(t_{5}\right)\left(t_{5}-\tau_{5}\right)\left(t_{5}-t_{4}\right)
$$

o que implica

$$
2 \leq\left(t_{5}-\tau_{5}\right)\left(t_{5}-t_{4}\right)=\left(t_{5}-\tau_{5}\right)^{2}+\left(t_{5}-\tau_{5}\right)\left(\tau_{5}-t_{4}\right) .
$$

Portanto, $t_{5}-\tau_{5} \geq 1$. 
Corolário 3.1 Suponhamos no sistema (3.6) que as funções $h_{j}, j=1,2$, sejam não decrescentes e satisfaçam a desigualdade $\left|h_{j}(z)\right| \leq \sqrt{2}|z|$. Então $t_{5}-t_{4} \geq 3 / 2$.

Prova A demonstração segue diretamente do Lema 3.2.

Lema 3.3 O operador $A: K \rightarrow K$ definido em (3.7) é completamente contínuo.

Para demonstrarmos o Lema 3.3 precisaremos do seguinte

Lema 3.4 Seja $h_{j}$ não decrescente, $j=1,2, G \subset C$ um subconjunto aberto e $0 \in G$. Então existe uma vizinhança $V$ de 0 em $C$ tal que para toda $\varphi \in V \cap K, \varphi \neq 0$, tem-se $x_{t}(\cdot ; \varphi) \in G$ para $0 \leq t \leq \tau(\varphi)$.

Prova Devido à simetria de nossas hipóteses, basta provarmos que existe uma vizinhança $V$ de 0 em $C$ tal que para toda $\varphi \in V \cap K, \varphi \neq 0$, tem-se $x_{t}(\cdot ; \varphi) \in G$ para $0 \leq t \leq t_{1}(\varphi)+1$. Para isso, provaremos que dado $G \subset \mathbb{R}^{2}$ aberto, $0 \in G$, existe uma vizinhança $V$ de 0 em $C$ tal que para toda $\varphi \in V \cap K, \varphi \neq 0$, tem-se $x(t ; \varphi) \in G$ para $-1 \leq t \leq t_{1}(\varphi)+1$

Como

$$
F(\varphi)=\left(\int_{-1}^{-1 / 2} h_{2}\left(\varphi_{2}(\theta)\right) d \theta,-\int_{-1}^{-1 / 2} h_{1}\left(\varphi_{1}(\theta)\right) d \theta\right)
$$

é contínua e $F(0)=0$, segue do teorema da continuidade em relação às condições iniciais que é suficiente provar a existência de uma vizinhança $V$ de 0 em $C$ tal que para toda $\varphi \in V \cap K, \varphi \neq 0$, tem-se $x(t ; \varphi) \in G$ para $-1 \leq t \leq t_{1}(\varphi)$.

Sejam $k, \ell$ números reais tais que

$$
\ell>k>0, \quad h_{2}(k)<\frac{\ell}{2} \quad \text { e } \quad R=\left\{\left(x_{1}, x_{2}\right) \mid 0 \leq x_{1} \leq \ell, 0 \leq x_{2} \leq k\right\} \subset G .
$$

Diminua $k$, se necessário, de modo que

$$
\frac{h_{2}(k)}{h_{1}(\ell / 4)}<\frac{\ell}{2 k}
$$


Escolha $V$ tal que se $\varphi \in V \cap K, \varphi \neq 0$, então $\varphi_{1}(\theta) \leq \ell / 4$ e $\varphi_{2}(\theta) \leq \min \{\ell / 4, k\}$, para $\theta \in[-1,0]$.

Tome $\varphi \in V \cap K, \varphi \neq 0$. Seja $t_{0}$ tal que $x_{1}\left(t_{0}, \varphi\right)=\ell / 4$ (se não existir $t_{0}$ nada restará a provar). Aplicando o Teorema do Valor Médio a $x_{1}$ no intervalo $\left[t_{0}, t_{0}+1\right]$ temos que existe $t^{\prime} \in\left(t_{0}, t_{0}+1\right)$ tal que

$$
x_{1}\left(t_{0}+1 ; \varphi\right)-x_{1}\left(t_{0} ; \varphi\right)=\int_{t^{\prime}-1}^{t^{\prime}-1 / 2} h_{2}\left(x_{2}(s)\right) d s \leq \int_{t^{\prime}-1}^{t^{\prime}-1 / 2} h_{2}(k) d s=\frac{1}{2} h_{2}(k)<\frac{\ell}{4}
$$

pois $h_{2}$ é não decrescente e $x_{2}(s) \leq k$, para $t^{\prime}-1 \leq s \leq t^{\prime}-1 / 2$. Seja $\bar{t}$ tal que $x_{1}(\bar{t} ; \varphi)=\ell / 2$ (se não existir $\bar{t}$ nada restará a provar).

Para $t \geq \bar{t}$ e $x(t)$ em $Q_{1}$, temos $x_{1}(s) \geq x_{1}(\bar{t}-1)>\ell / 4$ se $t-1 \leq s \leq t-1 / 2$, o que implica

$$
\left|\dot{x}_{2}(t)\right|=\left|\int_{t-1}^{t-1 / 2} h_{1}\left(x_{1}(s)\right) d s\right| \geq \frac{1}{2} h_{1}(\ell / 4),
$$

pois $h_{1}$ é não decrescente. Também

$$
\dot{x}_{1}(t)=\int_{t-1}^{t-1 / 2} h_{2}\left(x_{2}(s)\right) d s \leq \frac{1}{2} h_{2}(k) .
$$

Temos que o vetor tangente $\left(\dot{x}_{1}(t), \dot{x}_{2}(t)\right)$, satisfaz para $t \geq \bar{t}$

$$
\frac{\dot{x}_{1}(t)}{\left|\dot{x}_{2}(t)\right|} \leq \frac{h_{2}(k)}{h_{1}(\ell / 4)}<\frac{\ell}{2 k} .
$$

Portanto $x(t, \varphi) \in G$ para $-1 \leq t \leq t_{1}(\varphi)$, pois $x_{1}(t ; \varphi) \leq \ell$ e $x_{2}(t ; \varphi) \leq k$.

Prova do Lema 3.3. A continuidade da solução em relação às condições iniciais garante a continuidade de $A$ em $K \backslash\{0\}$. A continuidade de $A$ em $\varphi=0$ segue do Lema 3.4.

Seja $B \subset C$ limitado, $0 \in B$. Assim $V=B \cap K$ é limitado em $K$ e $0 \in V$. Seja

$$
A V=\left\{x_{\tau(\varphi)}(\cdot ; \varphi) \mid \varphi \in V \backslash\{0\}\right\} \cup\{0\}
$$


Temos que $A V \subset K$. Vamos mostrar que $A V$ é relativamente compacto. Devido à simetria de nossas hipóteses, basta mostrarmos que

$$
A_{1} V=\left\{x_{t_{1}(\varphi)+1}(\cdot \varphi) \mid \varphi \in V \backslash\{0\}\right\} \cup\{0\}
$$

Mostremos primeiro que $A_{1} V$ é limitado. Para isso, vamos mostrar que dado $W \subset \mathbb{R}^{2}$ limitado, $0 \in W$, existe um retângulo $R$, que depende só de $W$, tal que se $\varphi(\theta) \in W$, $\theta \in[-1,0]$, então $x(t ; \varphi) \in R$ para $-1 \leq t \leq t_{1}(\varphi)$. Provada essa afirmação e repetindo quatro vezes os argumentos teremos que existe um limitado $L \subset \mathbb{R}^{2}$ tal que se $\varphi(\theta) \in W, \theta \in[-1,0]$, então $x(t, \varphi) \in L,-1 \leq t \leq \tau(\varphi)$.

Seja $\varphi \in K$ tal que $\varphi(\theta) \in W, \theta \in[-1,0]$. Vamos mostrar que existe $\ell>0$, $\ell$ dependendo só de $W$, tal que se $\varphi(\theta) \in W, \theta \in[-1,0]$, então $x_{1}\left(t_{1}(\varphi) ; \varphi\right) \leq \ell$. Observe que se $\varphi(\theta) \in W, \theta \in[-1,0]$ e $W$ é limitado, então $x_{2}(t ; \varphi)$ é limitado para $-1 \leq t \leq t_{1}(\varphi)$. Na verdade, $x_{2}(t ; \varphi) \leq x_{2}(0 ; \varphi)$ para $0 \leq t \leq t_{1}(\varphi)$.

Como $\varphi(\theta) \in W, \theta \in[-1,0]$, existem $k, k^{\prime}$ números reais tais que $\varphi_{2}(\theta) \leq k$, $\theta \in[-1,0]$ e $\varphi_{1}(0) \leq k^{\prime}$. Tome $n$ suficientemente grande tal que se $\varphi \in V$ então

$$
\varphi(\theta) \in[0, n / 4] \times[0, k], \quad h_{2}(k)<n / 2 \quad \text { e } \frac{h_{2}(k)}{h_{1}(n / 4)}<\frac{n}{2 k} .
$$

Adaptações óbvias dos argumentos da prova do Lema 3.4 mostram que

$$
x_{1}(t ; \varphi) \leq n \quad \text { para } \quad-1 \leq t \leq t_{1}(\varphi)
$$

e, observe que, $x_{2}(t ; \varphi) \leq k$ para $\quad-1 \leq t \leq t_{1}(\varphi)$.

Logo, dado $W \subset \mathbb{R}^{2}$ limitado, existem constantes $M_{1}, M_{2}$ positivas, tais que

$$
\left|x_{1}(t ; \varphi)\right| \leq M_{1} \quad \text { e } \quad\left|x_{2}(t ; \varphi)\right| \leq M_{2}
$$

para $0 \leq t \leq \tau(\varphi)$, se $\varphi(\theta) \in W, \theta \in[-1,0]$. Mostremos agora que $A V$ é eqüicontínuo. Como $h_{1}$ e $h_{2}$ são contínuas, temos

(i) $\left|x_{1}(t ; \varphi)\right| \leq M_{1}$ implica $\left|h_{1}\left(x_{1}(s)\right)\right| \leq \min \left\{h_{1}\left(M_{1}\right),-h_{1}\left(-M_{1}\right)\right\} \stackrel{\text { def }}{=} 2 N_{2}$

(ii) $\left|x_{2}(t ; \varphi)\right| \leq M_{2}$ implica $\left|h_{2}\left(x_{2}(s)\right)\right| \leq \min \left\{h_{2}\left(M_{2}\right),-h_{2}\left(-M_{2}\right)\right\} \stackrel{\text { def }}{=} 2 N_{1}$. 
Assim

$$
\left|\dot{x}_{1}(t)\right| \leq N_{1} \quad \text { e } \quad\left|\dot{x}_{2}(t)\right| \leq N_{2}
$$

Então

$\left|A \varphi\left(\theta_{1}\right)-A \varphi\left(\theta_{2}\right)\right| \leq|\dot{x}(\hat{t} ; \varphi)|\left|\left(\tau(\varphi)+\theta_{1}\right)-\left(\tau(\varphi)+\theta_{2}\right)\right|=|\dot{x}(\hat{t} ; \varphi)|\left|\theta_{1}-\theta_{2}\right| \leq N\left|\theta_{1}-\theta_{2}\right|$

onde $\hat{t} \in\left[\tau(\varphi)+\theta_{1}, \tau(\varphi)+\theta_{2}\right]$ e $N$ depende de $N_{1}$ e $N_{2}$. Logo $A V$ é eqüicontínuo. Pelo teorema de Ascoli-Arzela temos que $A V$ é relativamente compacto, o que completa a prova.

Da prova do Lema 3.3 segue imediatamente o seguinte

Corolário 3.2 O operador A é limitado, isto é, A leva conjuntos limitados em conjuntos limitados.

Definição 3.4 Seja $\varphi \in K \backslash\{0\}$. O arco $x(t ; \varphi),-1 \leq t \leq t_{4}(\varphi)$, determina com os eixos coordenados, $x_{1}$ e $x_{2}$, uma região limitada em cada quadrante, $Q_{1}, Q_{2}, Q_{3}, Q_{4}$. Se cada uma dessas regiões for convexa, diremos que $x(t ; \varphi)$ é uma solução convexa.

Observação 3.2 Se $\varphi \in K \backslash\{0\}$, com a função $\varphi_{1}$ crescente, então a curva $x(t ; \varphi)$, $-1 \leq t \leq t_{4}(\varphi)$, define $x_{2}$ como função de $x_{1}$ nos quadrantes $Q_{1}$ e $Q_{3}$ e $x_{1}$ como função de $x_{2}$ nos quadrantes $Q_{2}$ e $Q_{4}$. Se as funções em $Q_{1}$ e $Q_{2}$ forem côncavas e as funções em $Q_{3}$ e $Q_{4}$ forem convexas, a solução $x(t ; \varphi)$ será convexa.

A Figura 3.1 mostra um esboço de uma solução $x(t ; \varphi)$ convexa.

Os próximos lemas conduzem à ejetividade da origem para o operador $A: K \rightarrow K$. Provaremos no Lema 3.5 que, se $\varphi=A \psi$ para algum $\psi \in K \backslash\{0\}$ então $x(t ; \varphi)$ é uma solução convexa e, portanto, $x\left(t ; A^{n} \varphi\right)$ é convexa. Também usaremos a notação $\tau_{i+1}(\psi), i=1,2,3,4, \psi \in K \backslash\{0\}$ dada na Definição 3.3.

Lema 3.5 Suponhamos no sistema (3.6), que as funções $h_{j}$ sejam crescentes e $\left|h_{j}(z)\right| \leq$ $\sqrt{2}|z|, j=1,2$. Se $\varphi=A \psi$ para algum $\psi \in K \backslash\{0\}$, então $x(t ; \varphi)$ é convexa. 
Prova Mostremos que $x(t ; \varphi)$ define $x_{2}$ como função côncava de $x_{1}$ em $Q_{1}$. A prova é baseada no estudo do vetor tangente $\left(\dot{x}_{1}(t), \dot{x}_{2}(t)\right)$.

Observe que $x(t ; \varphi),-1 \leq t \leq t_{1}(\varphi)$ coincide com $x(t ; \psi), t_{4}(\psi) \leq t \leq t_{5}(\psi)$. Assim, é suficiente provar que $x(t, \psi)$ define $x_{2}$ como função côncava de $x_{1}$ para $t_{4}(\psi) \leq t \leq t_{5}(\psi)$. Para $t \in\left(t_{4}(\psi), \tau_{5}(\psi)\right)$, a variável de integração $s$ em (3.6) fica restrita ao intervalo $\left[t_{4}(\psi)-1, \tau_{5}(\psi)-1 / 2\right]$. Como $t_{4}(\psi)-\tau_{4}(\psi) \geq 1$ e $\tau_{5}(\psi)-t_{4}(\psi) \geq$ $1 / 2$ temos que $x_{1}$ e $x_{2}$ são crescentes no intervalo $\left[t_{4}(\psi)-1, \tau_{5}(\psi)-1 / 2\right]$ e, portanto, $h_{1}\left(x_{1}\right)$ e $h_{2}\left(x_{2}\right)$ são crescentes nesse intervalo. Assim $\dot{x_{1}}(t)$ cresce e $\dot{x_{2}}(t)$ decresce no intervalo $\left[t_{4}(\psi), \tau_{5}(\psi)\right]$, ou seja, $x_{2}$ é uma função côncava de $x_{1}$ nesse trecho.

Para $\tau_{5}(\psi)<t<\tau_{5}(\psi)+1 / 2$ a variável $s$ no sistema (3.6) fica restrita ao intervalo $\left[\tau_{5}(\psi)-1, \tau_{5}(\psi)\right]$. Como $t_{4}(\psi)-\tau_{4}(\psi) \geq 1$ e $\tau_{5}(\psi)-t_{4}(\psi) \geq 1 / 2$ temos que $x_{1}$ e $x_{2}$ são crescentes no intervalo $\left[\tau_{5}(\psi)-1, \tau_{5}(\psi)\right]$ e, portanto, $h_{1}\left(x_{1}\right)$ e $h_{2}\left(x_{2}\right)$ são crescentes nesse intervalo. Logo $\dot{x_{1}}(t)$ cresce e $\dot{x_{2}}(t)$ decresce no intervalo $\left[\tau_{5}(\psi), \tau_{5}(\psi)+1 / 2\right]$.

Para $\tau_{5}(\psi)+1 / 2<t<\tau_{5}(\psi)+1$ temos $\tau_{5}(\psi)-1 / 2<s<\tau_{5}(\psi)+1 / 2$. Se $\tau_{5}(\psi)-\frac{1}{2}<s<\tau_{5}(\psi)$ temos que $x_{1}$ e $x_{2}$ são crescentes, o que implica $h_{1}\left(x_{1}\right)$ e $h_{2}\left(x_{2}\right)$ crescentes. Assim $\dot{x_{1}}(t)$ cresce e $\dot{x_{2}}(t)$ decresce. Se $\tau_{5}(\psi)<s<\tau_{5}(\psi)+1 / 2$ temos que $x_{1}$ cresce e $x_{2}$ decresce. Então, $\dot{x_{1}}(t)$ e $\dot{x_{2}}(t)$ decrescem.

Para $\tau_{5}(\psi)+1<t<t_{5}(\psi)$ temos $\tau_{5}(\psi)<s<t_{5}(\psi)-1 / 2$. Logo, temos $x_{1}$ crescente e $x_{2}$ decrescente. Então, $h_{1}\left(x_{1}\right)$ é crescente e $h_{2}\left(x_{2}\right)$ decrescente, o que implica que $\dot{x_{1}}(t)$ e $\dot{x_{2}}(t)$ decrescem.

Logo, $x(t ; \psi), t_{4}(\psi) \leq t \leq t_{5}(\psi)$, define $x_{2}$ como uma função côncava de $x_{1}$.

Procedendo de modo análogo nos outros quadrantes, concluímos que $x(t ; \varphi)$ é convexa.

Lema 3.6 Se as funções $h_{j}$ são crescentes, $\left|h_{j}(z)\right| \leq \sqrt{2}|z|, h_{j} \in C^{2}$ numa vizinhança de $0, j=1,2$, e satisfazem $0<h_{1}^{\prime}(0)=h_{2}^{\prime}(0) \leq \sqrt{2}, j=1,2$, temos que existe $\delta>0$ tal que se $\varphi=A \psi, \psi \in K \backslash\{0\},\|\varphi\|<\delta$ então $x(t ; \varphi) \cdot \dot{x}(t ; \varphi)>0, t \geq 0$, enquanto $|x(t ; \varphi)|<\delta$. 
Prova Seja $h_{j}^{\prime}(0)=\nu, j=1,2$. Então $h_{j}\left(x_{j}(s)\right)=\nu x_{j}(s)+R_{j}\left(x_{j}(s)\right)$ onde $R_{j}(y)=$ $O\left(y^{2}\right)$ quando $y \rightarrow 0$. Denotando $y=\left(y_{1}, y_{2}\right)$ e $R(y)=\left(R_{1}\left(y_{1}\right), R_{2}\left(y_{2}\right)\right)$, teremos

$$
\begin{gathered}
x(t) \cdot \dot{x}(t)=\left(x_{1}(t), x_{2}(t)\right) \cdot\left(\int_{t-1}^{t-1 / 2} h_{2}\left(x_{2}(s)\right) d s,-\int_{t-1}^{t-1 / 2} h_{1}\left(x_{1}(s)\right) d s\right)= \\
=\int_{t-1}^{t-\frac{1}{2}}\left[x_{1}(t) h_{2}\left(x_{2}(s)\right)-x_{2}(t) h_{1}\left(x_{1}(s)\right)\right] d s= \\
=\int_{t-1}^{t-1 / 2}\left(x_{1}(t), x_{2}(t)\right) \cdot\left(h_{1}\left(x_{1}(s)\right), h_{2}\left(x_{2}(s)\right)\right)^{\perp} d s= \\
=\int_{t-1}^{t-1 / 2}\left(x_{1}(t), x_{2}(t)\right) \cdot\left(\nu x_{2}(s)+R_{2}\left(x_{2}(s)\right),-\nu x_{1}(s)-R_{1}\left(x_{1}(s)\right) d s=\right. \\
=\int_{t-1}^{t-1 / 2}\left[\nu\left(x_{1}(t), x_{2}(t)\right) \cdot\left(x_{2}(s),-x_{1}(s)\right)+\left(x_{1}(t), x_{2}(t)\right) \cdot\left(R_{2}\left(x_{2}(s)\right),-R_{1}\left(x_{1}(s)\right)\right)\right] d s= \\
=\int_{t-1}^{t-1 / 2}\left[\nu\left(x(t) \cdot x^{\perp}(s)\right)+x(t) \cdot R^{\perp}(x(s))\right] d s .
\end{gathered}
$$

Como

$$
x(t) \cdot x^{\perp}(s)=O(|x(t)||x(s)|) \quad \text { e } \quad x(t) \cdot R^{\perp}(x(s))=O\left(|x(t)||x(s)|^{2}\right),
$$

$\operatorname{com}|x(t)|,|x(s)| \rightarrow 0$, o sinal de $x(t) \cdot \dot{x}(t)$ é o sinal de $\int_{t-1}^{t-1 / 2}\left[x(t) \cdot x^{\perp}(s)\right] d s$, ou seja, $x(t) \cdot \dot{x}(t)>0$.

Teorema 3.2 Se as funções $h_{j}$ são crescentes, $\left|h_{j}(z)\right| \leq \sqrt{2}|z|, h_{j} \in C^{2}$ numa vizinhança de $0, j=1,2$, e satisfazem $0<h_{1}^{\prime}(0)=h_{2}^{\prime}(0) \leq \sqrt{2}$, então 0 é ponto ejetivo do operador $A$.

Prova Seja $\delta>0$ fornecido pelo Lema 3.6. De acordo com o Lema 3.4, existe uma vizinhança $V$ de 0 em $C, V=B_{\delta^{\prime}}=\left\{\varphi \in C \mid\|\varphi\|<\delta^{\prime}\right\}$, tal que se $\varphi \in V \cap K, \varphi \neq 0$ então $x(t ; \varphi) \in B_{\delta}, t \in[-1, \tau(\varphi)], B_{\delta} \subset \mathbb{R}^{2}$.

Tomemos $\varphi \in V \cap K \backslash\{0\}$ e provemos que existe $m=m(\varphi)$ tal que $A^{m} \varphi \notin V \cap K$. 
Se $A \varphi \notin V$ não há o que provar. Então podemos supor que $A \varphi \in V$ e, neste caso, podemos tomar $A \varphi$ como condição inicial, isto é, não há perda de generalidade em supor que $\varphi=A \psi$, para algum $\psi \in K \backslash\{0\}$. Pelo Lema $3.6|x(t ; \varphi)|$ é crescente para $-1 \leq t \leq \tau(\varphi)$. Então existe $t_{0}>0$ tal que $x\left(t_{0} ; \varphi\right)=\delta^{\prime}$, ou seja, existe $m=m(\varphi)$ tal que $A^{m} \varphi \notin V$.

Suponhamos que as funções $h_{j}: \mathbb{R} \rightarrow \mathbb{R}, j=1,2$, dadas em (3.3) satisfaçam as seguintes condições:

(i) são contínuas,

(ii) $z h_{j}(z)>0$ para $z \neq 0$,

(iii) são crescentes,

(iv) $\left|h_{j}(z)\right| \leq \sqrt{2}|z|$,

(v) $h_{j} \in C^{2}$ numa vizinhança de 0 ,

(vi) $0<h_{1}^{\prime}(0)=h_{2}^{\prime}(0) \leq \sqrt{2}$ e

(vii) existem números reais $\ell_{1}>0, \ell_{2}>0$, tais que $h_{1}(z) \geq-\ell_{1}$ e $h_{2}(z) \leq \ell_{2}$, para todo $z$.

Sejam $c>0$ e $\Lambda_{1} \stackrel{\text { def }}{=} \sqrt{\left.\left(c+\ell_{1} / 2\right)\right)^{2}+\ell_{2}^{2} / 4}$. Definamos $W$ o subconjunto de $\mathbb{R}^{2}$ dado por

$$
W=\left\{x \in \mathbb{R}^{2}|| x \mid \leq \Lambda_{1}\right\} \cap Q_{1},
$$

e $V$ o subconjunto de $C=C\left([-1,0], \mathbb{R}^{2}\right)$ dado por

$$
V=\{\varphi \in C \mid \varphi(\theta) \in W, \theta \in[-1,0]\}
$$

Tomemos $\varphi \in V \cap K \backslash\{0\}$.

Afirmação: Existe um retângulo $R$ tal que $x(t ; \varphi) \in R,-1 \leq t \leq t_{1}(\varphi)$. 
Observemos que $x_{2}(t ; \varphi) \leq \Lambda_{1}$ para $-1 \leq t \leq t_{1}(\varphi)$ e $\varphi_{1}(0) \leq \Lambda_{1}$. Tomemos $n_{1} \geq 4 \Lambda_{1}$ suficientemente grande de modo que

$$
h_{2}\left(\Lambda_{1}\right)<\frac{n_{1}}{2} \quad \text { e } \quad \frac{h_{2}\left(\Lambda_{1}\right)}{h_{1}\left(n_{1} / 4\right)}<\frac{n_{1}}{2 \Lambda_{1}} .
$$

Os argumentos da prova do Lema 3.3 implicam $x_{1}\left(t_{1}(\varphi) ; \varphi\right) \leq n_{1}$. Portanto,

$$
0 \leq x_{1}(t ; \varphi) \leq n_{1} \quad \text { e } \quad 0 \leq x_{2}(t ; \varphi) \leq \Lambda_{1}, \quad t \in\left[-1, t_{1}(\varphi)\right]
$$

e está provada nossa afirmação.

Observemos também que

$\dot{x}_{1}\left(t_{1}(\varphi)+t ; \varphi\right) \leq \frac{1}{2} h_{2}\left(\Lambda_{1}\right)<\frac{n_{1}}{4} \quad$ e $\quad\left|\dot{x}_{2}\left(t_{1}(\varphi)+t ; \varphi\right)\right| \leq \frac{1}{2} h_{1}\left(n_{1}+n_{1} / 4\right), \quad t \in[0,1]$.

Vamos refazer em $Q_{2}, Q_{3}$ e $Q_{4}$ o raciocínio realizado acima.

Seja $\Lambda_{2} \stackrel{\text { def }}{=} \sqrt{\left(n_{1}+n_{1} / 4\right)^{2}+h_{1}\left(n_{1}+n_{1} / 4\right)^{2} / 4}$. Escolhamos $n_{2} \geq 4 \Lambda_{2}$ suficientemente grande de modo que

$$
h_{1}\left(\Lambda_{2}\right)<\frac{n_{2}}{2} \quad \text { e } \quad \frac{h_{1}\left(\Lambda_{2}\right)}{\left|h_{2}\left(-n_{2} / 4\right)\right|}<\frac{n_{2}}{2 \Lambda_{2}} .
$$

Então

$$
\begin{gathered}
-n_{2} \leq x_{2}\left(t_{2}(\varphi) ; \varphi\right) \leq 0, \quad 0 \leq x_{1}(t ; \varphi) \leq n_{1}+n_{1} / 4, \quad t \in\left[t_{1}, t_{2}\right], \\
\left|\dot{x}_{1}\left(t_{2}(\varphi)+t ; \varphi\right)\right| \leq \frac{1}{2}\left|h_{2}\left(-n_{2}-n_{2} / 4\right)\right| \quad \text { e } \quad\left|\dot{x}_{2}\left(t_{2}(\varphi)+t ; \varphi\right)\right|<\frac{n_{2}}{4}, \quad t \in[0,1] .
\end{gathered}
$$

Seja $\Lambda_{3} \stackrel{\text { def }}{=} \sqrt{\left(n_{2}+n_{2} / 4\right)^{2}+h_{2}\left(-n_{2}-n_{2} / 4\right)^{2} / 4}$. Escolhamos $n_{3} \geq 4 \Lambda_{3}$ suficientemente grande de modo que

$$
\left|h_{2}\left(-\Lambda_{3}\right)\right|<\frac{n_{3}}{2} \quad \text { e } \quad \frac{\left|h_{2}\left(-\Lambda_{3}\right)\right|}{\left|h_{1}\left(-n_{3} / 4\right)\right|}<\frac{n_{3}}{2 \Lambda_{3}} .
$$

Então

$$
\begin{gathered}
-n_{3} \leq x_{1}\left(t_{3}(\varphi) ; \varphi\right) \leq 0, \quad-\left(n_{2}+n_{2} / 4\right) \leq x_{2}(t ; \varphi) \leq 0, \quad t \in\left[t_{2}, t_{3}\right] \\
\left|\dot{x}_{1}\left(t_{3}(\varphi)+t ; \varphi\right)\right|<\frac{n_{3}}{4} \quad \text { e } \quad\left|\dot{x}_{2}\left(t_{3}(\varphi)+t ; \varphi\right)\right| \leq \frac{1}{2}\left|h_{1}\left(-n_{3}-n_{3} / 4\right)\right|, \quad t \in[0,1] .
\end{gathered}
$$


Seja $\Lambda_{4} \stackrel{\text { def }}{=} \sqrt{\left(n_{3}+n_{3} / 4\right)^{2}+h_{1}\left(-n_{3}-n_{3} / 4\right)^{2} / 4}$. Escolhamos $n_{4} \geq 4 \Lambda_{4}$ suficientemente grande de modo que

$$
\left|h_{1}\left(-\Lambda_{4}\right)\right|<\frac{n_{4}}{2} \quad \text { e } \quad \frac{\left|h_{1}\left(-\Lambda_{4}\right)\right|}{\left|h_{2}\left(n_{4} / 4\right)\right|}<\frac{n_{4}}{2 \Lambda_{4}} .
$$

Então

$$
\begin{gathered}
0 \leq x_{2}\left(t_{4}(\varphi) ; \varphi\right) \leq n_{4}, \quad-\left(n_{3}+n_{3} / 4\right) \leq x_{1}(t ; \varphi) \leq 0, \quad t \in\left[t_{3}, t_{4}\right] \\
\dot{x}_{1}\left(t_{4}(\varphi)+t ; \varphi\right) \leq \frac{1}{2} h_{2}\left(n_{4}+n_{4} / 4\right) \quad \text { e } \quad \dot{x}_{2}\left(t_{4}(\varphi)+t ; \varphi\right) \leq \frac{1}{2}\left|h_{1}\left(-\Lambda_{4}\right)\right|<\frac{n_{4}}{4}, \quad t \in[0,1] .
\end{gathered}
$$

Retomemos o sistema impulsivo de auto-sustentação (3.1),(3.2) com as funções $h_{j}: \mathbb{R} \rightarrow \mathbb{R}, j=1,2$, satisfazendo as seguintes condições:

(i) são contínuas,

(ii) $z h_{j}(z)>0$ para $z \neq 0$,

(iii) são crescentes,

(iv) $\left|h_{j}(z)\right| \leq \sqrt{2}|z|$,

(v) $h_{j} \in C^{2}$ numa vizinhança de 0 ,

(vi) $0<h_{1}^{\prime}(0)=h_{2}^{\prime}(0) \leq \sqrt{2}$,

(vii) existem números reais $\ell_{1}>0, \ell_{2}>0$, tais que $h_{1}(z) \geq-\ell_{1}$ e $h_{2}(z) \leq \ell_{2}$, para todo $z$

e a constante $\gamma$ em (3.2) no intervalo $\left[1, n_{4} /\left(n_{4}-c\right)\right]$.

Lembremos que nosso objetivo é investigar a existência de soluções periódicas lentamente espiralantes do sistema (3.1),(3.2). Um contexto natural para esse estudo é a teoria das equações diferenciais funcionais nos espaços $L^{p}$ (aqui tomaremos $p=1$ ) cujos fatos relevantes para este trabalho estão apresentados no Capítulo 1. 
No que segue estendemos de modo óbvio a definição de $F(\varphi)$, de $\varphi \in C\left([-1,0], \mathbb{R}^{2}\right)$ para $\varphi \in L^{1}\left([-1,0], \mathbb{R}^{2}\right)$ (na verdade necessitamos apenas da extensão para $\varphi$ seccionalmente contínua).

Como os valores de uma solução nos instantes de impulso são imateriais, podemos reformular o problema (3.1),(3.2) de modo a torná-lo adequado ao contexto do Capítulo 1 do seguinte modo:

$$
\begin{gathered}
\dot{x}(t)=F\left(x_{t}\right) \\
x\left(t^{-}\right)=\left(0, x_{2}\left(t^{-}\right)\right), x_{2}\left(t^{-}\right) \geq c \Rightarrow x(t)=\left(0, x_{2}\left(t^{-}\right)-\gamma\left(x_{2}\left(t^{-}\right)-c\right)\right) .
\end{gathered}
$$

Se $\hat{t}$ é um instante de impulso e $[\hat{t}, \hat{t}+\delta), \delta>0$, é um intervalo onde não ocorrem novos impulsos, a solução do problema (3.9),(3.10) para $t \in[\hat{t}, \hat{t}+\delta)$ é entendida como a solução do seguinte problema de valor inicial em $L^{1}$ :

$$
\left\{\begin{aligned}
\dot{x}(t) & =\bar{F}\left({ }^{t} x\right) \\
{ }^{t} x & =\left(x_{\hat{t}}, \xi\right)
\end{aligned}\right.
$$

onde $\xi=x\left(\hat{t}^{-}\right)-\gamma\left(x\left(\hat{t}^{-}\right)-(0, c)\right)$ e $\bar{F}(\varphi, \xi)=F(\varphi)$. Isto é, $x(t)=\xi+\int_{\hat{t}}^{t} F\left(x_{s}\right) d s$.

Consideremos o subconjunto fechado, limitado, convexo de $C$,

$$
\mathcal{K}=K \cap \bar{B}_{\Lambda_{1}}
$$

onde $\bar{B}_{\Lambda_{1}}=\left\{\varphi \in C \mid\|\varphi\| \leq \Lambda_{1}\right\}$ e $\Lambda_{1}$ foi definida anteriormente.

Definamos em $\mathcal{K}$ o operador de retorno $\mathcal{A}$,

$$
\mathcal{A} \varphi= \begin{cases}A \varphi, & \text { se } \quad \varphi=0 \quad \text { ou } \quad x_{2}\left(t_{4}^{-}(\varphi) ; \varphi\right)<c, \\ x_{t_{4}(\varphi)+1}(\cdot ; \varphi, \xi), & \text { se } \quad x_{2}\left(t_{4}^{-}(\varphi) ; \varphi\right) \geq c,\end{cases}
$$

onde $\xi=x\left(t_{4}^{-}(\varphi) ; \varphi\right)-\gamma\left(x\left(t_{4}^{-}(\varphi) ; \varphi\right)-(0, c)\right)$.

De acordo com as considerações anteriores, é óbvio que $\mathcal{A K} \subset \mathcal{K}$. Além disso, definindo

$$
K_{\Lambda}=K \cap \bar{B}_{\Lambda}, \quad \Lambda>0,
$$


temos

$$
\left.\mathcal{A}\right|_{K_{\Lambda}}=\left.A\right|_{K_{\Lambda}}
$$

para $\Lambda>0$ suficientemente pequeno, de acordo com o Lema 3.4. Assim 0 é ponto ejetivo de $\mathcal{A}$. De acordo com o Teorema $1.4, \mathcal{A}: \mathcal{K} \rightarrow \mathcal{K}$ é contínuo. Na verdade, $\mathcal{A}$ é completamente contínuo, uma vez que $\mathcal{A K}$ é relativamente compacto.

Estes argumentos combinados com o Teorema 3.1 constituem a prova do seguinte

Teorema 3.3 Suponhamos que as seguintes hipóteses estejam satisfeitas para as funções $h_{j}, j=1,2$, em (3.3):

(i) são contínuas,

(ii) $z h_{j}(z)>0$ para $z \neq 0$,

(iii) são crescentes,

(iv) $\left|h_{j}(z)\right| \leq \sqrt{2}|z|$

(v) $h_{j} \in C^{2}$ numa vizinhança de 0 ,

(vi) $0<h_{1}^{\prime}(0)=h_{2}^{\prime}(0) \leq \sqrt{2} e$

(vii) existem números reais $\ell_{1}>0, \ell_{2}>0$, tais que $h_{1}(z) \geq-\ell_{1}$ e $h_{2}(z) \leq \ell_{2}$, para todo $z$.

Então existe uma solução periódica não trivial lentamente espiralante do problema impulsivo de auto-sustentação (3.9),(3.10).

\subsection{Comentários finais}

Os argumentos apresentados até aqui não garantem a existência de soluções periódicas lentamente espiralantes impulsivas de (3.9),(3.10). Mais precisamente, o Teorema 3.3 não assegura que as soluções dadas pelos pontos fixos do operador $\mathcal{A}$ sofram impulsos. 
Afirmamos entretanto que, se as hipóteses do Teorema 3.3 estão satisfeitas, é possível escolher a constante $c$ no problema (3.9),(3.10) de modo que qualquer ponto fixo do operador $\mathcal{A}$ dê origem a uma solução impulsiva.

De fato, suponhamos que exista uma seqüência $\varphi_{n} \in \mathcal{K}$ tal que

$$
\mathcal{A} \varphi_{n}=\varphi_{n} \quad \text { e } \quad x_{2}\left(t_{4}^{n}\right) \stackrel{\text { def }}{=} x_{2}\left(t_{4}\left(\varphi_{n}\right) ; \varphi_{n}\right) \rightarrow 0, \quad \text { quando } \quad n \rightarrow \infty
$$

Sabemos que $\tau_{5}^{n}$, dado na Definição 3.3, pertence ao intervalo $\left(t_{4}^{n}+1 / 2, t_{4}^{n}+1\right]$. Aplicando o Teorema do Valor Médio a $x_{2}$ no intervalo $\left[t_{4}^{n}, \tau_{5}^{n}\right]$ e a $x_{1}$ no intervalo $\left[t_{4}^{n}-1, t_{4}^{n}\right]$ obtemos

$$
x_{2}\left(\tau_{5}^{n}\right)-x_{2}\left(t_{4}^{n}\right)<-\frac{1}{2} h_{1}\left(x_{1}\left(t_{4}^{n}-1\right) \quad \text { e } \quad x_{1}\left(t_{4}^{n}-1\right) \geq-\frac{1}{2} h_{2}\left(x_{2}\left(t_{4}^{n}\right)\right) .\right.
$$

Logo,

$$
-h_{1}\left(x_{1}\left(t_{4}^{n}-1\right) \leq-h_{1}\left((-1 / 2) h_{2}\left(x_{2}\left(t_{4}^{n}\right)\right)\right)\right.
$$

Assim,

$$
x_{2}\left(\tau_{5}^{n}\right)-x_{2}\left(t_{4}^{n}\right) \rightarrow 0, \quad \text { quando } \quad n \rightarrow \infty
$$

Também, aplicando o Teorema do Valor Médio a $x_{1}$ no intervalo $\left[t_{4}^{n}, t_{4}^{n}+1\right]$ obtemos

$$
x_{1}\left(t_{4}^{n}+1\right) \leq \frac{1}{2} h_{2}\left(x_{2}\left(\tau_{5}^{n}\right)\right) \text {. }
$$

Como $x_{2}\left(\tau_{5}^{n}\right) \rightarrow 0$ quando $n \rightarrow \infty$ temos $x_{1}\left(t_{4}^{n}+1\right) \rightarrow 0$ quando $n \rightarrow \infty$.

Portanto, para $n$ suficientemente grande, $\mathcal{A} \varphi_{n}$ está na região de ejetividade da origem, o que contraria a periodicidade de $x\left(\cdot ; \varphi_{n}\right)$. Isto significa que existe uma cota inferior positiva $\bar{c}$ para os números $x_{2}\left(t_{4}(\varphi) ; \varphi\right), \operatorname{com} \varphi \in \mathcal{K}$ e $\mathcal{A} \varphi=\varphi$.

Tomando-se $c, 0<c<\bar{c}$, o problema (3.9),(3.10) tem soluções periódicas lentamente espiralantes impulsivas. 


\section{Referências Bibliográficas}

[1] M. Baptistini e P.Z. Táboas, On the stability of some exponential polynomials, Journal of Mathematical Analysis and Applications, Vol. 205, 259-272 (1997).

[2] R.G. Bartle, Elements of Integration, New York, John Wiley (1966).

[3] H. Brezis, Analyse Functionnelle, Masson Editeur (1983).

[4] L.A.V. Carvalho, On a method to investigate bifurcation of periodic solutions in retarded differential equations, J. of Difference Equations and Applications, Vol. 4, 17-27 (1998).

[5] J.K. Hale, Theory of Functional Differential Equations, New York, Springer (1977).

[6] V. Lakshmikantham, D.D. Bainov e P.S. Simeonov, Theory of Impulsive Differential Equations, World Scientific Publishing Company, Singapore (1989).

[7] A.D. Myshkis, Auto-oscillations in continuous systems with impulsive selfsupport, Resenhas do Instituto de Matemática e Estatística da Universidade de São Paulo, Vol. 3, N. 1, 93-106 (1997).

[8] A.D. Myshkis, Autonomous differential equations with impulsive self-support and infinite delay, Funct. Differ. Equats. 3, 145-154 (1995).

[9] R.D. Nussbaum, Periodic solutions of nonlinear autonomous functional differential equations, Lecture Notes in Mathematics 730, Berlin, Springer (1979). 
[10] R.D. Nussbaum, Periodic solutions of some nonlinear autonomous functional differential equations, Ann. Mat. Pura Appl, Vol. 101, 263-306 (1974).

[11] J.C.F. de Oliveira e L.A.V. Carvalho, A Lyapunov functional for a retarded differential equation, SIAM J. Math. Anal, Vol.16, N.6, 1295-1305 (1985).

[12] J.C.F. de Oliveira e L. Fichmann, Discontinuous solutions of neutral functional differential equations, Publicacions Matematiques, Vol. 37, 369-386 (1993).

[13] L. Pontryagin, On the zeros of some elementary transcedental functions, A.M.S. Translations(2)1, 95-110 (1995).

[14] F. Riesz e B. Nagy, Functional Analysis, New York, Frederick Ungar (1972).

[15] H.O. Walther, Über Ejektivität und Periodische Lösungen bei Autonomen Funktionaldifferentialgleichungen mit verteilter Verzögerung, Habilitationsschrift, Univ. München (1977).

[16] P.Z. Táboas, Periodic solutions of a planar delay equations, Proc. Royal Society Edinburgh, Vol. 116A, 85-101 (1990). 


\section{Índice Remissivo}

conjunto

fortemente contido, 11

equação característica, 48

ponto ejetivo, 58

solução

continuável, 26

convexa, 67

impulsiva, 75

periódica lentamente espiralante, 59

do problema de valor inicial, 5

teorema

da bifurcação de Hopf, 55

dependência contínua, 19

de existência, 17

de Fréchet-Kolmogorov, 11

de ponto fixo, 58

de Schauder, 16

unicidade, 24 